\title{
Expedient, High-Yielding Synthesis of Silyl-Substituted Salen Ligands
}

\author{
Avinash N. Thadani, Yong Huang, and Viresh H. Rawal ${ }^{\star}$ \\ Department of Chemistry, The University of Chicago \\ 5735 South Ellis Avenue, Chicago, Illinois 60637. \\ vrawal@uchicago.edu
}

\section{Supporting Information}

\section{Experimental Procedures and Characterization Data}

General. Chlorotrimethysilane and para-anisaldehyde were distilled prior to use. All other reagents were used as received (Aldrich, Acros). $\mathrm{Et}_{2} \mathrm{O}$ and $\mathrm{THF}$ were distilled over sodium/benzophenone ketal. Trimethylamine, diemethoxyethane and toluene were distilled over $\mathrm{CaH}_{2}$. Melting points were measured on a Fisher-Johns melting point apparatus and are uncorrected. ${ }^{1} \mathrm{H}$ and ${ }^{13} \mathrm{C}$ NMR were recorded at 400 or $500 \mathrm{MHz}$ and 100 or $125 \mathrm{MHz}$ respectively on a Bruker DRX-500 and 400 spectrometers. Proton chemical shifts were internally referenced to the residual proton resonance in $\mathrm{CDCl}_{3}(\delta$ 7.26). Carbon chemical shifts were internally referenced to the deuterated solvent signals in $\mathrm{CDCl}_{3}(\delta 77.00)$. Infrared spectra were obtained on a Nicolet 20 SXB FT-IR spectrometer. Silica gel (60 Å, 230-400 mesh) was obtained from Silicycle and used as received.

\section{General Procedure A: Synthesis of 2,6-Dibromotrimethylsilylphenol ethers (2a, 2g, 2j)}

A solution of the appropriate 2,6-dibromophenol $(10 \mathrm{mmol})^{1}$ in THF $(10 \mathrm{~mL})$ was cooled in an ice-bath and treated with triethylamine $(1.67 \mathrm{~mL}, 12.0 \mathrm{mmol})$ followed by dropwise addition of chlorotrimethysilane $(1.40 \mathrm{~mL}, 11.0 \mathrm{mmol})$. The ice-bath was removed, and the stirred mixture was allowed to warm up to rt over $2 \mathrm{~h}$. The solvent was removed in vacuo and the residue diluted with hexanes $(100 \mathrm{~mL})$. The mixture was then filtered over Celite ${ }^{\circledR}$ and the residue washed with hexanes $(30 \mathrm{~mL})$. The filtrate was concentrated in vacuo to afford the silylether products as clear, colorless oils. The 2,6-dibromotrimethylsilylphenol ethers $(\mathbf{2} \mathbf{a}, \mathbf{2 g}, \mathbf{2 j})$ prepared by this method were used directly in General Procedure C.

${ }^{1}$ 2,6-Dibromo-4-methylphenol, 2,6-dibromophenol and 2,6-dibromo-4-fluorophenol were obtained from either Aldrich or Acros. 2,6-Dibromo-4-tert-butylphenol was prepared using a reported procedure (Lam, F.; Chan, K.S. Tetrahedron Lett. 1995, 36, 919-922). 


\section{General Procedure B: Synthesis of all other 2,6-Dibromosilylphenol ethers (2b-f, 2h-i)}

A solution of the 2,6-dibromophenol ${ }^{2}(5 \mathrm{mmol})$ and imidazole $(1.36 \mathrm{~g}, 20.0 \mathrm{mmol})$ in $\mathrm{CH}_{2} \mathrm{Cl}_{2}(5$ $\mathrm{mL}$ ) was cooled in an ice-bath and then treated with the required silylchloride $(5 \mathrm{mmol})$. The reaction mixture was heated to reflux for $16 \mathrm{~h}$. The solvent was then removed in vacuo and the residue diluted with hexanes $(100 \mathrm{~mL})$ and water $(50 \mathrm{~mL})$. The layers were separated and the organic layer washed with aqueous $\mathrm{HCl}(0.2 \mathrm{~N}, 30 \mathrm{~mL})$, water $(30 \mathrm{~mL})$, and brine $(30 \mathrm{~mL})$. The solution was then dried over $\mathrm{MgSO}_{4}$ and concentrated in vacuo. Compounds $\mathbf{2 b}, \mathbf{2 e}, \mathbf{2 g}$, and $\mathbf{2 i}$ were used directly in General Procedure C. Compounds 2c, 2d, 2f, 2h silyl ethers were purified by flash chromatography on silica gel, and then subjected to General Procedure C.

\section{(2,6-dibromo-4-methylphenoxy)triisopropylsilane (2c)}

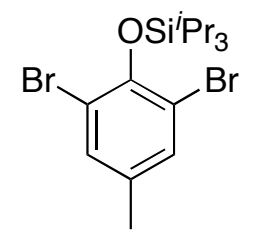

2c

Silyl ether 2c was isolated as a clear, colorless crystalline solid ( $82 \%$ yield): $R_{\mathrm{f}}=0.61(10 \%$ benzene/90\% hexanes); $\mathrm{mp}=95{ }^{\circ} \mathrm{C}$ (hexanes); ${ }^{1} \mathrm{H} \mathrm{NMR}\left(\mathrm{CDCl}_{3}, 500 \mathrm{MHz}\right) \delta 7.27(2 \mathrm{H}, \mathrm{s}), 2.23$ $(3 \mathrm{H}, \mathrm{s}), 1.57(3 \mathrm{H}$, septet, $J=7.5 \mathrm{~Hz}), 1.17(18 \mathrm{H}, \mathrm{d}, J=7.5 \mathrm{~Hz}) ;{ }^{13} \mathrm{C} \mathrm{NMR}\left(\mathrm{CDCl}_{3}, 125 \mathrm{MHz}\right) \delta$ 148.99, 133.06, 132.71, 115.34, 19.86, 18.07, 14.41; IR (film) v 3020, 2945, 2867, 1539, 1467, $1386,1284,1249,1196,1064,1017,998,920,885,852,736,719,684,657 \mathrm{~cm}^{-1}$.

2 2,6-Dibromo-4-methylphenol, 2,6-dibromophenol and 2,6-dibromo-4-fluorophenol were obtained from either Aldrich or Acros.

Note 2: 2,6-Dibromo-4-tert-butylphenol was prepared using a reported procedure (Lam, F.; Chan, K.S. Tetrahedron Lett. 1995, 36, 919-922). 


\section{(2,6-dibromo-4-methylphenoxy)(1,1-dimethylethyl)diphenyl silane (2d)}

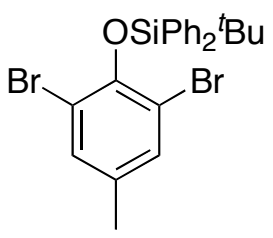

2d

Silyl ether 2d was isolated as a clear, colorless crystalline solid (87\% yield): $\mathrm{R}_{\mathrm{f}}=0.44(10 \%$ benzene/90\% hexanes); $\mathrm{mp}=79{ }^{\circ} \mathrm{C}$ (hexanes); ${ }^{1} \mathrm{H} \mathrm{NMR}\left(\mathrm{CDCl}_{3}, 500 \mathrm{MHz}\right) \delta 7.95(4 \mathrm{H}, \mathrm{d}, J=$ $7.0 \mathrm{~Hz}), 7.54-7.49(2 \mathrm{H}, \mathrm{m}), 7.46(4 \mathrm{H}, \mathrm{t}, J=7.5 \mathrm{~Hz}), 7.32(2 \mathrm{H}, \mathrm{s}), 2.27(3 \mathrm{H}, \mathrm{s}), 1.32(9 \mathrm{H}, \mathrm{s}) ;{ }^{13} \mathrm{C}$ NMR $\left(\mathrm{CDCl}_{3}, 125 \mathrm{MHz}\right) \delta 147.84,135.09,133.79,133.32,132.94,129.50,127.29,114.64$, 26.70, 20.40, $19.76 \mathrm{~cm}^{-1}$; IR (film) v 3072, 3048, 2930, 2893, 2858, 1465, 1428, 1389, 1289, $1115,927,872,821,740,700,652,605 \mathrm{~cm}^{-1}$.

\section{(2,6-Dibromophenoxy)methyldiphenylsilane (2f)}

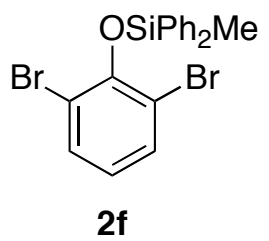

2f isolated as a clear, colorless crystalline solid (84\% yield): $\mathrm{R}_{\mathrm{f}}=0.50$ (10\% benzene/90\% hexanes); $\mathrm{mp}=70{ }^{\circ} \mathrm{C}$ (benzene); ${ }^{1} \mathrm{H} \mathrm{NMR}\left(\mathrm{CDCl}_{3}, 500 \mathrm{MHz}\right) \delta 7.82-7.74(4 \mathrm{H}, \mathrm{m}), 7.51-7.40$ $(8 \mathrm{H}, \mathrm{m}), 6.70(1 \mathrm{H}, \mathrm{t}, J=8.0 \mathrm{~Hz}), 1.00(3 \mathrm{H}, \mathrm{s}) ;{ }^{13} \mathrm{C} \mathrm{NMR}\left(\mathrm{CDCl}_{3}, 500 \mathrm{MHz}\right) \delta 150.52,134.77$, 134.66, 132.35, 130.01, 127.57, 123.69, 116.31, -0.65; IR (film) v 3066, 3012, 2980, 1588, $1551,1442,1427,1256,1113,1069,905,776,762,735,697 \mathrm{~cm}^{-1}$.

\section{(1,1-dimethylethyl)[2,6-dibromo-4-(1,1-dimethylethyl)phenoxy]diphenylsilane}




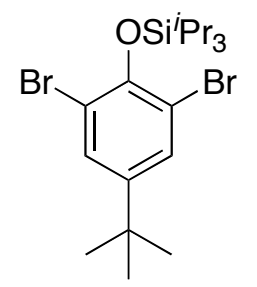

2i

Silyl ether $\mathbf{2} \mathbf{i}$ isolated as a clear, colorless crystalline solid (82\% yield): $\mathbf{R}_{\mathrm{f}}=0.57(10 \%$ benzene/90\% hexanes); $\mathrm{mp}=78{ }^{\circ} \mathrm{C}$ (hexanes); ${ }^{1} \mathrm{H} \mathrm{NMR}\left(\mathrm{CDCl}_{3}, 500 \mathrm{MHz}\right) \delta 7.45(2 \mathrm{H}, \mathrm{s}), 1.57$ $(3 \mathrm{H}$, septet, $J=7.5 \mathrm{~Hz}), 1.27(9 \mathrm{H}, \mathrm{s}), 1.16(18 \mathrm{H}, \mathrm{d}, J=7.5 \mathrm{~Hz}) ;{ }^{13} \mathrm{C} \mathrm{NMR}\left(\mathrm{CDCl}_{3}, 125 \mathrm{MHz}\right) \delta$ 148.78, 146.22, 129.78, 115.26, 34.21, 31.20, 18.09, 14.43; IR (film) v 3006, 2962, 2866, 2726, 1535, 1466, 1386, 1364, 1294, 1253, 1066, 1019, 917, 884, 861, 769, 373, 721, 686, $657 \mathrm{~cm}^{-1}$.

\section{General Procedure C: One-pot synthesis of 3-silylsubstituted salicylaldehydes (Table 2)}

A solution of 2,6-dibromophenol silylether $2(1.50 \mathrm{mmol})$ in $\mathrm{Et}_{2} \mathrm{O}(3.0 \mathrm{~mL})^{3}$ was cooled to -78 ${ }^{\circ} \mathrm{C}$ and treated dropwise with ${ }^{t} \mathrm{BuLi}(1.7 \mathrm{M}$ in pentane, $3.53 \mathrm{~mL}, 6.00 \mathrm{mmol})$. The reaction mixture was stirred for $1.5 \mathrm{~h}$ while warming to $0{ }^{\circ} \mathrm{C}$, and another $0.5 \mathrm{~h}$ at $0{ }^{\circ} \mathrm{C}$ (note 4 ). ${ }^{4}$ The mixture was then recooled to $-78{ }^{\circ} \mathrm{C}$ prior to the addition of DMF $(0.465 \mathrm{~mL}, 6.00 \mathrm{mmol})$, all at once. The reaction mixture was subsequently stirred for $1 \mathrm{~h}$ while warming to $0{ }^{\circ} \mathrm{C}$ and then quenched with saturated aq. $\mathrm{NH}_{4} \mathrm{Cl}(5.0 \mathrm{~mL})$ and diluted with $\mathrm{Et}_{2} \mathrm{O}(75 \mathrm{~mL})$. The layers were separated and the organic layer was washed with $\mathrm{H}_{2} \mathrm{O}(2 \times 15 \mathrm{~mL})$, brine $(25 \mathrm{~mL})$, dried $\left(\mathrm{MgSO}_{4}\right)$, and concentrated in vacuo to afford the crude salicylaldehyde. Flash chromatography (silica gel, EtOAc/hexanes or benzene/hexanes) afforded the pure salicyladehyde.

\section{2-Hydroxy-5-methyl-3-(trimethylsilyl)benzaldehyde (4a)}

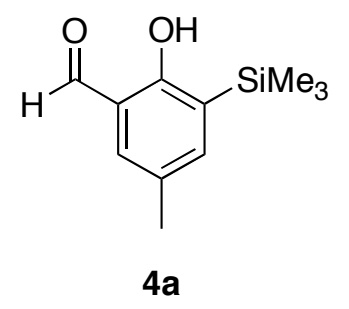

\footnotetext{
${ }^{3}$ The reactions of $\mathbf{2 d}, \mathbf{2} \mathbf{e}$ and $\mathbf{2 f}$ were conducted in THF (concentration: $0.5 \mathrm{M}$ ).
} 
Compound 4a was isolated as a clear, pale yellow crystalline solid: $\mathrm{R}_{\mathrm{f}}=0.31$ (15\% benzene $/ 85 \%$ hexanes); $\mathrm{mp}=61{ }^{\circ} \mathrm{C}$ (benzene); ${ }^{1} \mathrm{H} \mathrm{NMR}\left(\mathrm{CDCl}_{3}, 500 \mathrm{MHz}\right) \delta 11.16(1 \mathrm{H}, \mathrm{s}), 9.82(1 \mathrm{H}, \mathrm{s}), 7.43$ $(1 \mathrm{H}, \mathrm{d}, J=2.5 \mathrm{~Hz}), 7.31(1 \mathrm{H}, \mathrm{d}, J=2.5 \mathrm{~Hz}), 2.34(3 \mathrm{H}, \mathrm{s}), 0.34(9 \mathrm{H}, \mathrm{s}) ;{ }^{13} \mathrm{C} \mathrm{NMR}\left(\mathrm{CDCl}_{3}, 125\right.$ MHz) $\delta 196.72,164.25,143.63,134.49,128.46,128.36,119.14,20.23$, -1.31; IR (film) v 3150 (br), 2957, 2838, 2732, 1648, 1609, 1586, 1400, 1309, 1245, 1200, 1163, 983, 952, 885, 836, $793,772,691,627 \mathrm{~cm}^{-1}$.

\section{3-(tert-Butyldimethylsilyl)-2-hydroxy-5-methylbenzaldehyde (4b)}

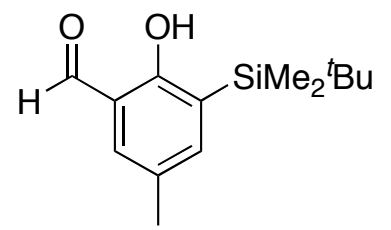

$4 b$

Compound 4b was isolated as a clear, colorless crystalline solid: $\mathrm{R}_{\mathrm{f}}=0.30$ (15\% benzene/85\% hexanes); $\mathrm{mp}=75^{\circ} \mathrm{C}$ (benzene); ${ }^{1} \mathrm{H} \mathrm{NMR}\left(\mathrm{CDCl}_{3}, 500 \mathrm{MHz}\right) \delta 11.23(1 \mathrm{H}, \mathrm{s}), 9.82(1 \mathrm{H}, \mathrm{s}), 7.43$ $(1 \mathrm{H}, \mathrm{d}, J=2.5 \mathrm{~Hz}), 7.31(1 \mathrm{H}, \mathrm{d}, J=2.5 \mathrm{~Hz}), 2.34(3 \mathrm{H}, \mathrm{s}), 0.93(9 \mathrm{H}, \mathrm{s}), 0.34(6 \mathrm{H}, \mathrm{s}) ;{ }^{13} \mathrm{C}$ NMR $\left(\mathrm{CDCl}_{3}, 125 \mathrm{MHz}\right) \delta 1$ 196.72, 164.55, 145.11, 134.77, 128.18, 126.23, 119.32, 26.93, 20.29, 17.47, -4.92, -4.94; IR (film) v 3200 (br), 3030, 2954, 2927, 2883, 2855, 1650, 1587, 1469, $1439,1409,1301,1248,1238,1163,963,878,837,772,758,700,674,666 \mathrm{~cm}^{-1}$.

\footnotetext{
${ }^{4}$ The reactions of $\mathbf{2 d}, \mathbf{2 e}$ and $\mathbf{2 f}$ were warmed to $\mathrm{rt}$ and stirred for $1 \mathrm{~h}$ at $\mathrm{rt}$.
} 


\section{2-Hydroxy-5-methyl-3-(triisopropylsilyl)benzaldehyde (4c)}

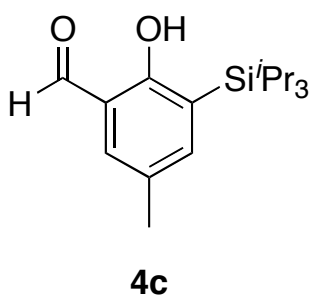

Compound 4c was isolated as a clear, colorless crystalline solid: $\mathrm{R}_{\mathrm{f}}=0.24$ (10\% benzene/90\% hexanes); $\mathrm{mp}=72{ }^{\circ} \mathrm{C}$ (benzene); ${ }^{1} \mathrm{H} \mathrm{NMR}\left(\mathrm{CDCl}_{3}, 500 \mathrm{MHz}\right) \delta 11.27(1 \mathrm{H}, \mathrm{s}), 9.83(1 \mathrm{H}, \mathrm{s}), 7.45$ $(1 \mathrm{H}, \mathrm{d}, J=2.0 \mathrm{~Hz}), 7.32(1 \mathrm{H}, \mathrm{d}, J=2.0 \mathrm{~Hz}), 2.34(3 \mathrm{H}, \mathrm{s}), 1.53(3 \mathrm{H}$, septet, $J=7.5 \mathrm{~Hz}), 1.09$ $(18 \mathrm{H}, \mathrm{d}, J=7.5 \mathrm{~Hz}) ;{ }^{13} \mathrm{C} \mathrm{NMR}\left(\mathrm{CDCl}_{3}, 125 \mathrm{MHz}\right) \delta 196.83,164.93,145.64,134.59,128.20$, 124.15, 119.34, 20.44, 18.79, 11.45; IR (film) v 3150 (br), 3030, 29945, 2865, 1650, 1586, 1463, $1302,1236,1200,883,864,771,750 \mathrm{~cm}^{-1}$.

\section{3-(tert-Butyldiphenylsilyl)-2-hydroxy-5-methylbenzaldehyde (4d)}<smiles>Cc1cc([SnH3])c(O)c(C=O)c1</smiles>

4d

Compound 4d was isolated as a clear, colorless crystalline solid: $\mathrm{R}_{\mathrm{f}}=0.28(40 \%$ benzene/60\% hexanes); $\mathrm{mp}=141{ }^{\circ} \mathrm{C}$ (softens at $\left.80{ }^{\circ} \mathrm{C}, \mathrm{CHCl}_{3}\right) ;{ }^{1} \mathrm{H} \mathrm{NMR}\left(\mathrm{CDCl}_{3}, 400 \mathrm{MHz}\right) \delta 11.49(1 \mathrm{H}, \mathrm{s})$, $9.91(1 \mathrm{H}, \mathrm{s}), 7.63-7.56(4 \mathrm{H}, \mathrm{m}), 7.48-7.37(7 \mathrm{H}, \mathrm{m}), 7.30(1 \mathrm{H}, \mathrm{d}, J=2.0 \mathrm{~Hz}), 2.24(3 \mathrm{H}, \mathrm{s})$, 1.26 ( 9H, s); ${ }^{13} \mathrm{C} \mathrm{NMR}\left(\mathrm{CDCl}_{3}, 100 \mathrm{MHz}\right) \delta 196.73,164.41,147.70,136.10,135.50,134.77$, 129.12, 128.65, 127.62, 123.95, 119.42, 29.62, 20.36, $18.52 \mathrm{~cm}^{-1}$; IR (film) v 3100 (br), 3070, 2960, 2905, 2858, 1652, 1586, 1428, 1300, 1267, 1240, 1197, 1164, 1105, 962, 867, 759, 741, $701 \mathrm{~cm}^{-1}$. 


\section{2-Bromo-6-(tert-butyldiphenylsilyl)-4-methylphenol (4dBr)}

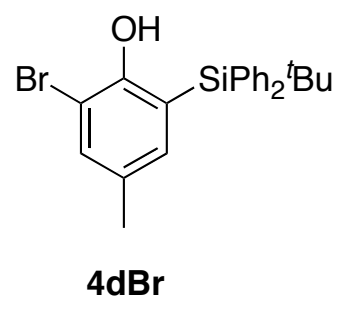

Compound $4 \mathbf{d B r}$ was isolated as clear, colorless crystalline solid: $\mathrm{R}_{\mathrm{f}}=0.50$ (40\% benzene/60\% hexanes); $\mathrm{mp}=78{ }^{\circ} \mathrm{C}\left(\mathrm{CHCl}_{3}\right) ;{ }^{1} \mathrm{H} \mathrm{NMR}\left(\mathrm{CDCl}_{3}, 500 \mathrm{MHz}\right) \delta 7.59(4 \mathrm{H}, \mathrm{dd}, J=8.0,1.5 \mathrm{~Hz})$, $7.47-7.37(7 \mathrm{H}, \mathrm{m}), 7.05(1 \mathrm{H}, \mathrm{d}, J=1.5 \mathrm{~Hz}), 5.69(1 \mathrm{H}, \mathrm{s}), 2.20(3 \mathrm{H}, \mathrm{s})), 1.25(9 \mathrm{H}, \mathrm{s}) ;{ }^{13} \mathrm{C} \mathrm{NMR}$ $\left(\mathrm{CDCl}_{3}, 125 \mathrm{MHz}\right) \delta 154.05,138.73,136.11,134.77,134.12,130.83,129.20,127.69,121.56$, 110.48, 29.53, 20.33, 18.65; IR (film) v 3505, 3069, 3049, 3027, 2996, 2932, 2890, 2857, 1589, 1563, 1486, 1471, 1449, 1427, 1391, 1363, 1320, 1254, 1237, 1213, 1174, 1105, 1076, 999, 903, $864,820,755,739,728,702,688,621,605,569 \mathrm{~cm}^{-1}$.

\section{2-Hydroxy-5-methyl-3-(triphenylsilyl)benzaldehyde (4e)}

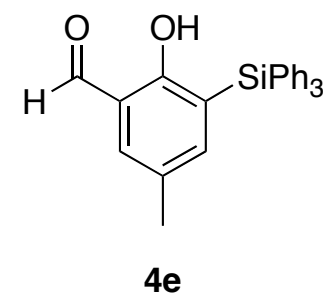

Compound 4e was isolated as a pale, yellow solid: $\mathrm{R}_{\mathrm{f}}=0.34$ (50\% benzene $/ 50 \%$ hexanes $)$; $\mathrm{mp}=$ $182{ }^{\circ} \mathrm{C}\left(\mathrm{CHCl}_{3}\right) ;{ }^{1} \mathrm{H} \mathrm{NMR}\left(\mathrm{CDCl}_{3}, 500 \mathrm{MHz}\right) \delta 11.36(1 \mathrm{H}, \mathrm{s}), 9.92(1 \mathrm{H}, \mathrm{s}), 7.71(6 \mathrm{H}, \mathrm{dd}, J=8.0$, $1.5 \mathrm{~Hz}), 7.53-7.44(10 \mathrm{H}, \mathrm{m}), 7.41(1 \mathrm{H}, \mathrm{d}, J=2.5 \mathrm{~Hz}), 2.30(3 \mathrm{H}, \mathrm{s}) ;{ }^{13} \mathrm{C} \mathrm{NMR}\left(\mathrm{CDCl}_{3}, 125\right.$ MHz) $\delta 196.55,164.44,146.87,136.21,135.91,133.80,129.49,128.87,127.75,123.06,119.61$, 20.28; IR (film) v 3200 (br), 3064, 3043, 2970, 1644, 1608, 1587, 1428, 1407, 1344, 1299, 1263, $1239,1201,1159,1106,958,872,759,745,700,676 \mathrm{~cm}^{-1}$. 
2-Hydroxy-3-(methyldiphenylsilyl)benzaldehyde (4f)

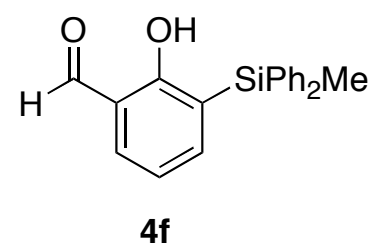

Compound $4 \mathbf{f}$ was isolated as a clear, colorless crystalline solid: $\mathrm{R}_{\mathrm{f}}=0.21(30 \%$ benzene/70\% hexanes); $\mathrm{mp}=80{ }^{\circ} \mathrm{C}\left(\mathrm{CHCl}_{3}\right) ;{ }^{1} \mathrm{H} \mathrm{NMR}\left(\mathrm{CDCl}_{3}, 500 \mathrm{MHz}\right) \delta 11.51(1 \mathrm{H}, \mathrm{s}), 9.93(1 \mathrm{H}, \mathrm{s}), 7.69-$ $7.63(5 \mathrm{H}, \mathrm{m}), 7.55-7.44(7 \mathrm{H}, \mathrm{m}), 7.03(1 \mathrm{H}, \mathrm{d}, J=7.5 \mathrm{~Hz}), 1.05(3 \mathrm{H}, \mathrm{s}) ;{ }^{13} \mathrm{C} \mathrm{NMR}\left(\mathrm{CDCl}_{3}, 125\right.$ MHz) $\delta 196.61,166.4,144.97,135.63,135.54,135.06,12.36,127.79,124.77,119.66,119.63$, 3.52; IR (film) v 3200 (br), 3068, 2850, 1653, 1603, 1575, 1486, 1420, 1384, 1295, 1251, 1218, $1111,1070,998,917,829,778,757,722,697,667 \mathrm{~cm}^{-1}$.

\section{2-Bromo-6-methyldiphenylsilylphenol (4fBr)}

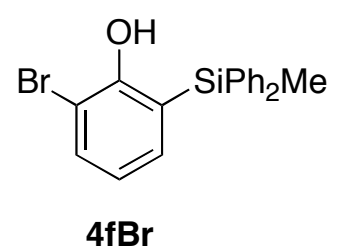

Compound $\mathbf{4 f B r}$ was isolated as a clear, colorless crystalline solid: $\mathrm{R}_{\mathrm{f}}=0.41$ (30\% benzene $/ 70 \%$ hexanes $), \mathrm{mp}=115^{\circ} \mathrm{C}\left(\mathrm{CHCl}_{3}\right) ;{ }^{1} \mathrm{H} \mathrm{NMR}\left(\mathrm{CDCl}_{3}, 500 \mathrm{MHz}\right) \delta 7.68-7.63(4 \mathrm{H}, \mathrm{m}), 7.61(1 \mathrm{H}$, $\mathrm{dd}, J=8.0,1.5 \mathrm{~Hz}), 7.54-7.45(6 \mathrm{H}, \mathrm{m}), 7.22(1 \mathrm{H}, \mathrm{dd}, J=7.5,1.5 \mathrm{~Hz}), 6.84(1 \mathrm{H}, \mathrm{t}, J=7.5 \mathrm{~Hz})$, $5.83(1 \mathrm{H}, \mathrm{s}), 1.03(3 \mathrm{H}, \mathrm{s}) ;{ }^{13} \mathrm{C} \mathrm{NMR}\left(\mathrm{CDCl}_{3}, 125 \mathrm{MHz}\right) \delta 156.40,136.94,135.70,135.08$, 133.86, 129.40, 127.82, 123.15, 121.66, 110.57, -3.37; IR (film) v 3484, 3067, 3041, 2970, $1583,1427,1319,1234,1107,1082,769,772,737,717,699 \mathrm{~cm}^{-1}$. 
5-tert-Butyl-2-hydroxy-3-(trimethylsilyl)benzaldehyde (4g)

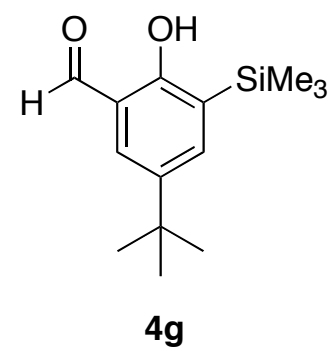

Compound $\mathbf{4 g}$ was isolated as a clear, colorless crystalline solid: $\mathrm{R}_{\mathrm{f}}=0.39$ (40\% benzene/60\% hexanes); $\mathrm{mp}=33{ }^{\circ} \mathrm{C}\left(\mathrm{CHCl}_{3}\right) ;{ }^{1} \mathrm{H} \mathrm{NMR}\left(\mathrm{CDCl}_{3}, 500 \mathrm{MHz}\right) \delta 11.21(1 \mathrm{H}, \mathrm{s}), 9.90(1 \mathrm{H}, \mathrm{s}), 7.70$ $(1 \mathrm{H}, \mathrm{d}, J=2.5 \mathrm{~Hz}), 7.53(1 \mathrm{H}, \mathrm{d}, J=2.5 \mathrm{~Hz}), 1.36(9 \mathrm{H}, \mathrm{s}) ;{ }^{13} \mathrm{C} \mathrm{NMR}\left(\mathrm{CDCl}_{3}, 125 \mathrm{MHz}\right) \delta$ 197.01, 164.23, 141.91, 140.05, 131.01, 128.00, 118.83, 34.05, 31.28, -1.26; IR (film) v 3100 (br), 3049, 2959, 2902, 2870, 1659, 1607, 1586, 1401, 1311, 1247, 1216, 952, 884, 839, 774, $735,700,635 \mathrm{~cm}^{-1}$.

5-tert-Butyl-3-(tert-butyldimethylsilyl)-2-hydroxybenzaldehyde (4h)

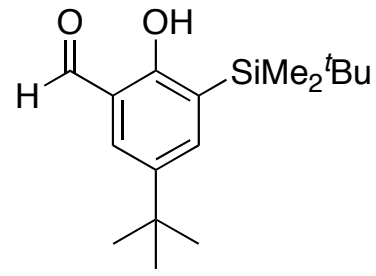

4h

Compound 4h was isolated as a clear, colorless crystalline solid: $\mathrm{R}_{\mathrm{f}}=0.23(10 \%$ benzene/90\% hexanes); $\mathrm{mp}=76{ }^{\circ} \mathrm{C}\left(\mathrm{CHCl}_{3}\right) ;{ }^{1} \mathrm{H} \mathrm{NMR}\left(\mathrm{CDCl}_{3}, 500 \mathrm{MHz}\right) \delta 11.28(1 \mathrm{H}, \mathrm{s}), 9.89(1 \mathrm{H}, \mathrm{s}), 7.71$ $(1 \mathrm{H}, \mathrm{d}, J=2.5 \mathrm{~Hz}), 7.52(1 \mathrm{H}, \mathrm{d}, J=2.5 \mathrm{~Hz}), 1.36(9 \mathrm{H}, \mathrm{s}), 0.93(9 \mathrm{H}, \mathrm{s}), 0.37(6 \mathrm{H}, \mathrm{s}) ;{ }^{13} \mathrm{C} \mathrm{NMR}$ $\left(\mathrm{CDCl}_{3}, 125 \mathrm{MHz}\right) \delta 1$ 196.99, 164.59, 141.84, 141.57, 131.07, 125.75, 118.98, 33.99, 31.24, 26.83, 17.47, -4.92; IR (film) v 3150 (br), 3050, 2952, 2851, 1642, 1605, 1584, 1458, 1393, $1360,1308,1260,1215,950,876,732,673 \mathrm{~cm}^{-1}$. 


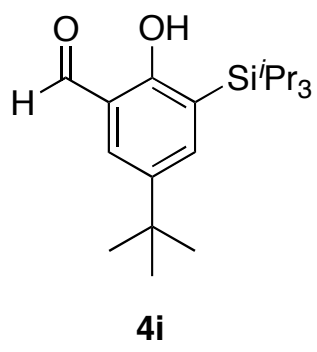

Compound 4i was isolated as a clear, colorless crystalline solid: $\mathrm{R}_{\mathrm{f}}=0.30$ (15\% benzene/85\% hexanes); $\mathrm{mp}=87{ }^{\circ} \mathrm{C}\left(\mathrm{CHCl}_{3}\right) ;{ }^{1} \mathrm{H} \mathrm{NMR}\left(\mathrm{CDCl}_{3}, 500 \mathrm{MHz}\right) \delta 11.35(1 \mathrm{H}, \mathrm{s}), 9.88(1 \mathrm{H}, \mathrm{s}), 7.75$ $(1 \mathrm{H}, \mathrm{d}, J=2.5 \mathrm{~Hz}), 7.50(1 \mathrm{H}, \mathrm{d}, J=2.5 \mathrm{~Hz}), 1.56(3 \mathrm{H}$, septet, $J=7.5 \mathrm{~Hz}), 1.35(9 \mathrm{H}, \mathrm{s}), 1.12$ $(18 \mathrm{H}, \mathrm{d}, J=7.5 \mathrm{~Hz}) ;{ }^{13} \mathrm{C} \mathrm{NMR}\left(\mathrm{CDCl}_{3}, 125 \mathrm{MHz}\right) \delta 196.99,165.00,142.67,141.48,130.75$, 123.48, 119.01, 33.97, 31.20, 18.76, 11.51; IR (film) v 3100 (br), 3030, 2943, 2863, 1643, 1604, $1582,1457,1399,1365,1307,1262,1216,949,880,862,760,645 \mathrm{~cm}^{-1}$.

\section{5-Fluoro-2-hydroxy-3-(trimethylsilyl)benzaldehyde (4j)}<smiles>C[AsH3]c1cc(F)cc(C=O)c1O</smiles>

4j

Compound 4j was isolated as a clear, colorless crystalline solid: $\mathrm{R}_{\mathrm{f}}=0.44$ (20\% benzene/80\% hexanes); $\mathrm{mp}=50{ }^{\circ} \mathrm{C}\left(\mathrm{CHCl}_{3}\right) ;{ }^{1} \mathrm{H} \mathrm{NMR}\left(\mathrm{CDCl}_{3}, 500 \mathrm{MHz}\right) \delta 11.10(1 \mathrm{H}, \mathrm{s}), 9.81(1 \mathrm{H}, \mathrm{s}), 7.32$ $(1 \mathrm{H}, \mathrm{dd}, J=8.0,3.0 \mathrm{~Hz}), 7.18(1 \mathrm{H}, \mathrm{dd}, J=7.5,3.0 \mathrm{~Hz}), 0.32(9 \mathrm{H}, \mathrm{s}) ;{ }^{13} \mathrm{C} \mathrm{NMR}\left(\mathrm{CDCl}_{3}, 125\right.$ MHz) $\delta 195.71$ (d, $J=2.0 \mathrm{~Hz}), 162.23,155.62$ (d, $J=242 \mathrm{~Hz}), 131.87$ (d, $J=2.5 \mathrm{~Hz}), 129.69$ (d, $J=22.0 \mathrm{~Hz}), 119.04(\mathrm{~d}, J=6.0 \mathrm{~Hz}), 118.69$ (d, $J=23.0 \mathrm{~Hz}),-1.57$; IR (film) v 3150 (br), 3062, 2958, 2901, 2854, 1660, 1613, 1584, 1423, 1373, 1301, 1240, 1181, 1126, 979, 893, 845, 800, $772,756,708 \mathrm{~cm}^{-1}$. 


\section{General Procedure D: Direct synthesis of silyl-substituted salens (Table 3)}

A solution of 2,6-dibromophenol silyl ether 2 (4.50 mmol) in $\mathrm{Et}_{2} \mathrm{O}(12.0 \mathrm{~mL})$ was cooled to -78 ${ }^{\circ} \mathrm{C}$ and treated dropwise with ${ }^{t} \mathrm{BuLi}(1.7 \mathrm{M}$ in pentane, $10.6 \mathrm{~mL}, 18.0 \mathrm{mmol})$. The reaction mixture was stirred for $1.5 \mathrm{~h}$ while warming to $0{ }^{\circ} \mathrm{C}$, and another $0.5 \mathrm{~h}$ at $0{ }^{\circ} \mathrm{C}$. The mixture was then recooled to $-78{ }^{\circ} \mathrm{C}$ prior to the addition of DMF $(1.40 \mathrm{~mL}, 18.0 \mathrm{mmol})$ all at once. The reaction mixture was subsequently stirred for $1 \mathrm{~h}$ while warming to $0{ }^{\circ} \mathrm{C}$ and then quenched with saturated aq. $\mathrm{NH}_{4} \mathrm{Cl}(15.0 \mathrm{~mL})$ and diluted with $\mathrm{Et}_{2} \mathrm{O}(200 \mathrm{~mL})$. The layers were separated and the organic layer washed with $\mathrm{H}_{2} \mathrm{O}(2 \times 30 \mathrm{~mL})$, brine $(50 \mathrm{~mL})$, dried $\left(\mathrm{MgSO}_{4}\right)$, and concentrated in vacuo to afford the crude salicylaldehyde (4).

A mixture of $(1 R, 2 R)-(+)-1,2$-diaminocyclohexane L-tartrate $(85.7 \mathrm{mg}, 0.75 \mathrm{mmol})$ and $\mathrm{K}_{2} \mathrm{CO}_{3}$ (207 mg, $1.50 \mathrm{mmol}$ ) was dissolved in $0.75 \mathrm{~mL} \mathrm{H} \mathrm{H}_{2} \mathrm{O}$. This solution was added to the crude silylsalicylaldehyde 4 dissolved in absolute ethanol $(15 \mathrm{~mL})$. The resultant yellow suspension was refluxed for 2 hours and $3.0 \mathrm{~mL} \mathrm{H}_{2} \mathrm{O}$ was added while hot. The reaction mixture was slowly cooled to room temperature, and the yellow precipitate was collected by filtration.

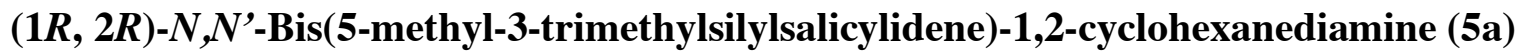

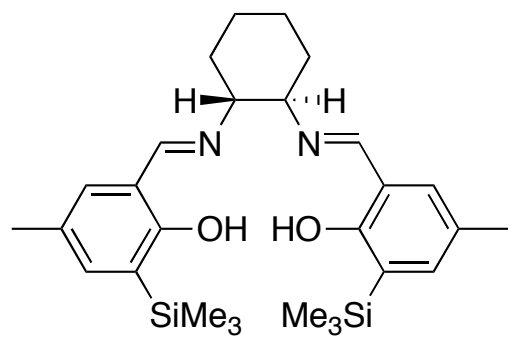

$5 a$

Salen 5a was isolated as a pale yellow, microcrystalline solid: $\mathrm{mp}=161{ }^{\circ} \mathrm{C}\left(\mathrm{EtOH} / \mathrm{H}_{2} \mathrm{O}\right) ;{ }^{1} \mathrm{H}$ NMR $\left(\mathrm{CDCl}_{3}, 500 \mathrm{MHz}\right) \delta 13.17(2 \mathrm{H}$, br s), $8.22(2 \mathrm{H}, \mathrm{s}), 7.13(2 \mathrm{H}, \mathrm{d}, J=2.0 \mathrm{~Hz}), 6.94(2 \mathrm{H}, \mathrm{d}, J$ $=2.0 \mathrm{~Hz}), 3.34-3.26(2 \mathrm{H}, \mathrm{m}), 2.22(6 \mathrm{H}, \mathrm{s}), 1.99-1.83(4 \mathrm{H}, \mathrm{m}), 1.80-1.68(2 \mathrm{H}, \mathrm{m}), 1.47(2 \mathrm{H}$, $\mathrm{t}, J=10.0 \mathrm{~Hz}), 0.31(18 \mathrm{H}, \mathrm{s}) ;{ }^{13} \mathrm{C} \mathrm{NMR}\left(\mathrm{CDCl}_{3}, 125 \mathrm{MHz}\right) \delta 165.04,163.46,138.24,132.93$, 126.94, 126.48, 117.23, 72.50, 33.08, 24.32, 20.27, -1.08; IR (film) v 3050 (br), 3030, 2980, $2935,2859,1657,1627,1585,1427,1374,1300,1244,1167,997,941,890,838,773,750,693$ $\mathrm{cm}^{-1}$. 


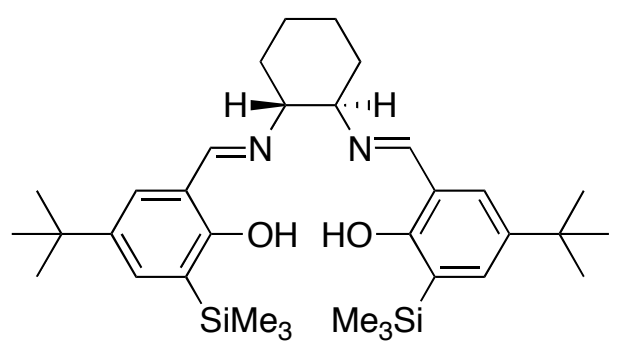

$5 b$

silyl5b was isolated as a pale yellow, microcrystalline solid: $\mathrm{mp}=191-193{ }^{\circ} \mathrm{C}\left(\mathrm{EtOH} / \mathrm{H}_{2} \mathrm{O}\right) ;{ }^{1} \mathrm{H}$ NMR $\left(\mathrm{CDCl}_{3}, 500 \mathrm{MHz}\right) \delta 13.23(2 \mathrm{H}$, br s), $8.28(2 \mathrm{H}, \mathrm{s}), 7.37(2 \mathrm{H}, \mathrm{d}, J=2.5 \mathrm{~Hz}), 7.13(2 \mathrm{H}, \mathrm{d}, J$ $=2.5 \mathrm{~Hz}), 3.36-3.27(2 \mathrm{H}, \mathrm{m}), 1.97-1.82(4 \mathrm{H}, \mathrm{m}), 1.79-1.67(2 \mathrm{H}, \mathrm{m}), 1.52-1.40(2 \mathrm{H}, \mathrm{m})$, $1.25(18 \mathrm{H}, \mathrm{s}), 0.30(18 \mathrm{H}, \mathrm{s}) ;{ }^{13} \mathrm{C} \mathrm{NMR}\left(\mathrm{CDCl}_{3}, 125 \mathrm{MHz}\right) \delta 165.28,163.47,140.35,134.64$, 129.35, 126.06, 116.86, 72.67, 33.87, 33.25, 31.39, 24.33, -1.02; IR (film) v 3010, 2962, 2903, $2863,1629,1584,1465,1432,1392,1363,1262,1246,971,886,839,773,750 \mathrm{~cm}^{-1}$.

(1R, 2R)-N,N'-Bis(5-tert-butyl-3-tert-butyldimethylsilylsalicylidene)-1,2cyclohexanediamine $(5 c)$

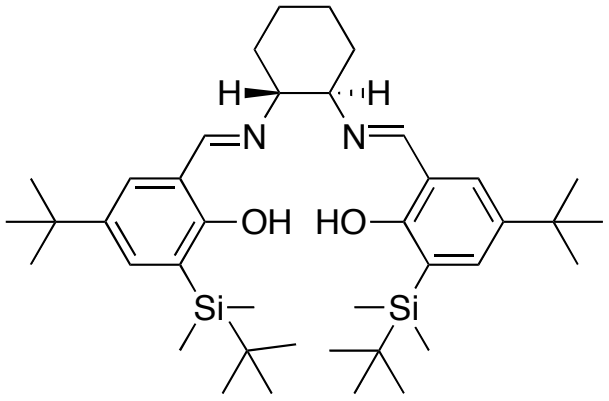

5c

Salen 5c was isolated as a pale yellow solid: $\mathrm{mp}=104-107{ }^{\circ} \mathrm{C}\left(\mathrm{EtOH} / \mathrm{H}_{2} \mathrm{O}\right) ;{ }^{1} \mathrm{H} \mathrm{NMR}\left(\mathrm{CDCl}_{3}\right.$, $500 \mathrm{MHz}) \delta 13.24(2 \mathrm{H}, \mathrm{s}), 8.26(2 \mathrm{H}, \mathrm{s}), 7.41(2 \mathrm{H}, \mathrm{d}, J=2.5 \mathrm{~Hz}), 7.12(2 \mathrm{H}, \mathrm{d}, J=2.5 \mathrm{~Hz}), 3.37$ $3.30(2 \mathrm{H}, \mathrm{m}), 2.00-1.85(4 \mathrm{H}, \mathrm{m}), 1.83-1.72(2 \mathrm{H}, \mathrm{m}), 1.48(2 \mathrm{H}, \mathrm{t}, J=9.5 \mathrm{~Hz}), 1.27(18 \mathrm{H}, \mathrm{s})$, 
$0.93(18 \mathrm{H}, \mathrm{s}), 0.35(12 \mathrm{H}, \mathrm{s}) ;{ }^{13} \mathrm{C} \mathrm{NMR}\left(\mathrm{CDCl}_{3}, 125 \mathrm{MHz}\right) \delta 165.36,163.79,139.89,136.42$, 129.31, 123.75, 116.97, 72.56, 33.80, 33.15, 31.39, 26.99, 24.34, 17.60, -4.71; IR (film) v 2957, 2859, 1629, 1521, 1471, 1432, 1363, 1032, 950, 906, 878, 837, 826, 773, $750 \mathrm{~cm}^{-1}$.

$(1 R, 2 R)-N, N^{\prime}$-Bis(5-tert-butyl-3-triisopropylsilylsalicylidene)-1,2-cyclohexanediamine (5d)

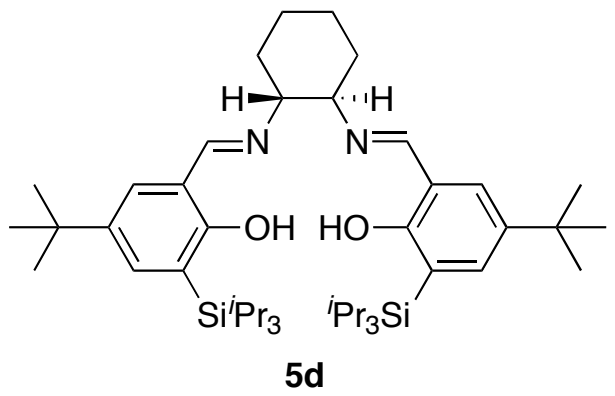

Salen 5d was isolated as a pale yellow solid: $\mathrm{mp}=199-207{ }^{\circ} \mathrm{C}\left(\mathrm{EtOH} / \mathrm{H}_{2} \mathrm{O}\right) ;{ }^{1} \mathrm{H} \mathrm{NMR}\left(\mathrm{CDCl}_{3}\right.$, $500 \mathrm{MHz}) \delta 13.30(2 \mathrm{H}$, br s), $8.18(2 \mathrm{H}, \mathrm{s}), 7.41(2 \mathrm{H}, \mathrm{d}, J=2.5 \mathrm{~Hz}), 7.03(2 \mathrm{H}, \mathrm{d}, J=2.5 \mathrm{~Hz})$, 3.33 - $3.26(2 \mathrm{H}, \mathrm{m}), 1.98-1.83(4 \mathrm{H}, \mathrm{m}), 1.81-1.70(2 \mathrm{H}, \mathrm{m}), 1.57-1.43(8 \mathrm{H}, \mathrm{m}), 1.22(18 \mathrm{H}$, s), $1.08(18 \mathrm{H}, \mathrm{d}, J=7.5 \mathrm{~Hz}) ; 1.07(18 \mathrm{H}, \mathrm{d}, J=7.5 \mathrm{~Hz}) ;{ }^{13} \mathrm{C} \mathrm{NMR}\left(\mathrm{CDCl}_{3}, 125 \mathrm{MHz}\right) \delta 165.50$, $164.12,139.69,137.21,128.88,121.40,116.83,72.54,33.75,33.07,31.34,24.33,18.92,18.89$, 11.69; IR (film) v 3010, 2956, 2944, 2864, 1628, 1464, 1432, 1264, 982, 950, 904, 881, 775, $771,840 \mathrm{~cm}^{-1}$.

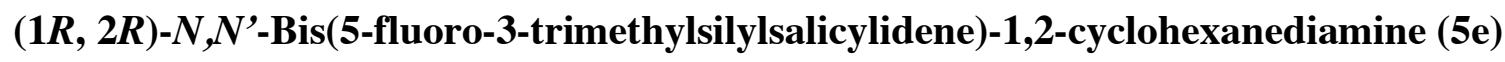

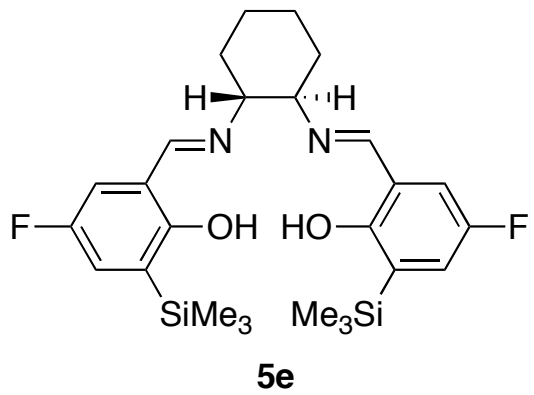

Salen 5e was isolated as a pale, yellow foamy solid: $\mathrm{mp}=64-66{ }^{\circ} \mathrm{C}\left(\mathrm{EtOH} / \mathrm{H}_{2} \mathrm{O}\right) ;{ }^{1} \mathrm{H}$ NMR $\left(\mathrm{CDCl}_{3}, 500 \mathrm{MHz}\right) \delta 13.08(2 \mathrm{H}, \mathrm{br} \mathrm{s}), 8.20(2 \mathrm{H}, \mathrm{s}), 7.03(2 \mathrm{H}, \mathrm{dd}, J=8.0,3.0 \mathrm{~Hz}), 6.81(2 \mathrm{H}, \mathrm{dd}$, 
$J=8.0,3.0 \mathrm{~Hz}), 3.37-3.29(2 \mathrm{H}, \mathrm{m}), 2.01-1.84(4 \mathrm{H}, \mathrm{m}), 1.80-1.68(2 \mathrm{H}, \mathrm{m}), 1.47(2 \mathrm{H}, \mathrm{t}, J=$ $10.0 \mathrm{~Hz}), 0.29(18 \mathrm{H}, \mathrm{s}) ;{ }^{13} \mathrm{C} \mathrm{NMR}\left(\mathrm{CDCl}_{3}, 125 \mathrm{MHz}\right) \delta 164.08(\mathrm{~d}, J=6.0 \mathrm{~Hz}), 161.47,155.29$, $129.38(\mathrm{~d}, J=3.5 \mathrm{~Hz}), 124.08(\mathrm{~d}, J=21 \mathrm{~Hz}), 117.50(\mathrm{~d}, J=23.0 \mathrm{~Hz}), 117.34(\mathrm{~d}, J=6.0 \mathrm{~Hz})$, 72.47, 32.90, 24.20, -1.34; IR (film) v 3090, 3005, 2936, 2900, 2860, 1632, 1582, 1448, 1427, $1377,1302,1239,1132,1095,989,941,918,896,867,839,789,773,753,695,629 \mathrm{~cm}^{-1}$.

\section{Methyl 2-Hydroxy-5-methyl-3-trimethylsilylbenzoate (6)}

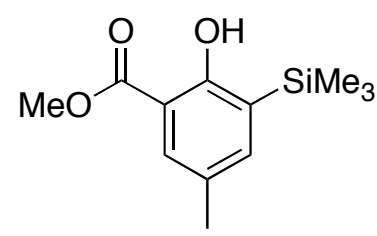

6

Salicylate ester 6 was prepared according to General Procedure $\mathrm{C}$ except that methylchloroformate $(464 \mu \mathrm{L}, 6.00 \mathrm{mmol})$ was added in place of DMF. Ester 6 was isolated as clear, colorless crystalline solid: $\mathrm{R}_{\mathrm{f}}=0.31$ (10\% EtOAc/90\% hexanes); $\mathrm{mp}=89{ }^{\circ} \mathrm{C}\left(\mathrm{CH}_{2} \mathrm{Cl}_{2}\right) ;{ }^{1} \mathrm{H}$ NMR $\left(\mathrm{CDCl}_{3}, 500 \mathrm{MHz}\right) \delta 10.85(1 \mathrm{H}, \mathrm{s}), 7.67-7.63(1 \mathrm{H}, \mathrm{m}), 7.37(1 \mathrm{H}, \mathrm{d}, J=2.0 \mathrm{~Hz}), 3.93$ (3H, s), $2.30(3 \mathrm{H}, \mathrm{s}), 0.34(9 \mathrm{H}, \mathrm{s}) ;{ }^{13} \mathrm{C} \mathrm{NMR}\left(\mathrm{CDCl}_{3}, 125 \mathrm{MHz}\right) \delta 170.97,164.18,142.27$, 130.73, 127.83, 127.64, 110.68, 52.06, 20.39, -1.22; IR (film) v 3150 (br), 2953, 2900, 2857, $1673,1605,1441,1406,1267,1246,1219,1117,1105,926,869,841,786,751,738,694 \mathrm{~cm}^{-1}$. 


\section{(2-Hydroxy-5-methyl-3-trimethylsilylphenyl)-4-methoxyphenylmethanone (7)}<smiles>COc1ccc(C(=O)c2cc(C)cc(C)c2O)cc1</smiles>

7

Ketone 7 was prepared according to General Procedure C except that para-anisaldehyde (730 $\mu \mathrm{L}, 6.00 \mathrm{mmol}$ ) was added in place of DMF. Ketone 7 was isolated as a clear, pale yellow crystalline solid: $\mathrm{R}_{\mathrm{f}}=0.43$ (50\% benzene $/ 50 \%$ hexanes); $\mathrm{mp}=94{ }^{\circ} \mathrm{C}\left(\mathrm{CHCl}_{3}\right) ;{ }^{1} \mathrm{H}$ NMR $\left(\mathrm{CDCl}_{3}\right.$, $500 \mathrm{MHz}) \delta 12.17(1 \mathrm{H}, \mathrm{s}), 7.69(2 \mathrm{H}, \mathrm{d}, J=9.0 \mathrm{~Hz}), 7.40(2 \mathrm{H}, \mathrm{s}), 7.00(2 \mathrm{H}, \mathrm{d}, J=9.0 \mathrm{~Hz}), 3.90$ $(3 \mathrm{H}, \mathrm{s}), 2.27(3 \mathrm{H}, \mathrm{s}), 0.35(9 \mathrm{H}, \mathrm{s}) ;{ }^{13} \mathrm{C} \mathrm{NMR}\left(\mathrm{CDCl}_{3}, 125 \mathrm{MHz}\right) \delta$ 200.42, 165.60, 162.60, 142.55, 134.17, 131.63, 130.78, 128.69, 126.95, 117.78, 113.54, 55.45, 20.56, -1.17; IR (film) $v$ 3200 (br), 3070, 3036, 2958, 2900, 2840, 1724, 1605, 1510, 1457, 1419, 1334, 1245, 1174, 1142, 1114, 1033, 1016, 982, 906, 843, 805, 754, 736, $696 \mathrm{~cm}^{-1}$.

\section{4'-Methoxy-5-methyl-3-trimethylsilylbiphenyl-2-ol (10)}

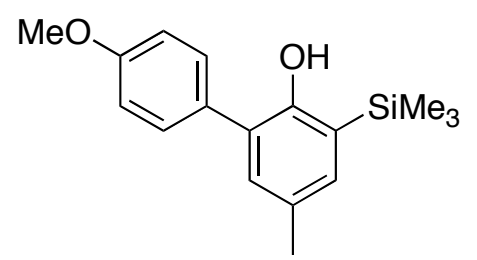

10

To a solution of $2 \mathrm{a}(1.50 \mathrm{mmol})$ in $\mathrm{Et}_{2} \mathrm{O}(3.0 \mathrm{~mL})$ cooled to $-78{ }^{\circ} \mathrm{C}$ was added ${ }^{t} \mathrm{BuLi}(1.7 \mathrm{M}$ in pentane, $3.53 \mathrm{~mL}, 6.00 \mathrm{mmol}$ ) dropwise. The reaction mixture was stirred for $1.5 \mathrm{~h}$ while warming to $0{ }^{\circ} \mathrm{C}$ and another $0.5 \mathrm{~h}$ at $0{ }^{\circ} \mathrm{C}$. The mixture was then recooled to $-78{ }^{\circ} \mathrm{C}$ prior to the addition of trimethylborate $(0.669 \mathrm{~mL}, 6.00 \mathrm{mmol})$ all at once. The reaction mixture was subsequently stirred for $1 \mathrm{~h}$ while warming to $0{ }^{\circ} \mathrm{C}$ and then quenched with $1 \mathrm{~N} \mathrm{HCl}(10 \mathrm{~mL})$ and diluted with $\mathrm{Et}_{2} \mathrm{O}(75 \mathrm{~mL})$. The layers were separated and the organic layer washed with $\mathrm{H}_{2} \mathrm{O}(2$ x $15 \mathrm{~mL})$, brine $(25 \mathrm{~mL})$, dried $\left(\mathrm{MgSO}_{4}\right)$, filtered and concentrated in vacuo to afford the crude 
boronic acid 8. Compound 8 was dissolved in a 1:1 mixture of toluene/DME $(3.0 \mathrm{~mL})$. 4Bromoanisole (9) (94 $\mu \mathrm{L}, 0.75 \mathrm{mmol})$ was added followed by $\mathrm{Cs}_{2} \mathrm{CO}_{3}(505 \mathrm{mg}, 3.00 \mathrm{mmol})$, and $\mathrm{Pd}\left(\mathrm{PPh}_{3}\right)_{4}(173 \mathrm{mg}, 0.15 \mathrm{mmol})$. The reaction mixture was subsequently heated at $80{ }^{\circ} \mathrm{C}$ for $6 \mathrm{~h}$. All volatiles were then removed in vacuo and the residue subjected to flash chromatography (silica gel).

Phenol 10 was isolated as a clear, colorless crystalline solid: $\mathrm{R}_{\mathrm{f}}=0.40(30 \%$ benzene $/ 70 \%$ hexanes); $\mathrm{mp}=95^{\circ} \mathrm{C}\left(\mathrm{CHCl}_{3}\right) ;{ }^{1} \mathrm{H} \mathrm{NMR}\left(\mathrm{CDCl}_{3}, 500 \mathrm{MHz}\right) \delta 7.41(2 \mathrm{H}, \mathrm{d}, J=9.0 \mathrm{~Hz}), 7.20$ $(1 \mathrm{H}, \mathrm{d}, J=2.0 \mathrm{~Hz}), 7.07(1 \mathrm{H}, \mathrm{d}, J=2.0 \mathrm{~Hz}), 7.05(2 \mathrm{H}, \mathrm{d}, J=9.0 \mathrm{~Hz}), 5.30(1 \mathrm{H}, \mathrm{s}), 3.88(3 \mathrm{H}, \mathrm{s})$, $2.36(3 \mathrm{H}, \mathrm{s}), 0.37(9 \mathrm{H}, \mathrm{s}) ;{ }^{13} \mathrm{C} \mathrm{NMR}\left(\mathrm{CDCl}_{3}, 125 \mathrm{MHz}\right) \delta 159.25,154.96,134.84,131.93$, $130.35,129.41,129.13,126.59,125.32,114.77,55.30,20.50$, -0.90; IR (film) v 3444, 3012, 2953, 2900, 1607, 1507, 1443, 1405, 1324, 1285, 1240, 1226, 1196, 1175, 1080, 1032, 916, 856, $829,785,752,730 \mathrm{~cm}^{-1}$.

\section{5-Methyl-3-trimethylsilylbiphenyl-2-ol (11)}

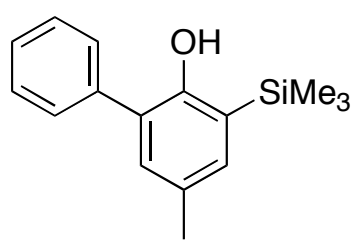

11

To a solution of $\mathbf{2 a}(1.50 \mathrm{mmol})$ in $\mathrm{Et}_{2} \mathrm{O}(3.0 \mathrm{~mL})$ cooled to $-78{ }^{\circ} \mathrm{C}$ was added ${ }^{t} \mathrm{BuLi}(1.7 \mathrm{M}$ in pentane, $3.53 \mathrm{~mL}, 6.00 \mathrm{mmol}$ ) dropwise. The reaction mixture was stirred for $1.5 \mathrm{~h}$ while warming to $0{ }^{\circ} \mathrm{C}$ and another $0.5 \mathrm{~h}$ at $0{ }^{\circ} \mathrm{C}$. The mixture was then recooled to $-78{ }^{\circ} \mathrm{C}$ prior to the dropwise addition of $\mathrm{ZnCl}_{2}\left(1.0 \mathrm{M}\right.$ in $\left.\mathrm{Et}_{2} \mathrm{O}, 6.00 \mathrm{~mL}, 6.00 \mathrm{mmol}\right)$. The reaction mixture was subsequently stirred for $1 \mathrm{~h}$ while warming to $-20{ }^{\circ} \mathrm{C}$. Bromobenzene $(316 \mu \mathrm{L}, 3.00 \mathrm{mmol})$ was added followed by $\mathrm{CuI}(5.8 \mathrm{mg}, 0.03 \mathrm{mmol})$ and $\mathrm{PdCl}_{2}\left(\mathrm{PPh}_{3}\right)_{2}(31.6 \mathrm{mg}, 0.045 \mathrm{mmol})$. The reaction mixture was slowly warmed to $50{ }^{\circ} \mathrm{C}$ over a period of $1 \mathrm{~h}$ and then kept at $50{ }^{\circ} \mathrm{C}$ for $7 \mathrm{~h}$. All volatiles were then removed in vacuo and the residue subjected to flash chromatography (silica gel). 
Phenol 11 was isolated as clear, colorless oil: $\mathrm{R}_{\mathrm{f}}=0.48$ (30\% benzene/70\% hexanes); ${ }^{1} \mathrm{H}$ NMR $\left(\mathrm{CDCl}_{3}, 500 \mathrm{MHz}\right) \delta 7.53-7.45(4 \mathrm{H}, \mathrm{m}), 7.41(1 \mathrm{H}, \mathrm{tt}, J=7.0,1.5 \mathrm{~Hz}), 7.20(1 \mathrm{H}, \mathrm{d}, J=2.0 \mathrm{~Hz})$, $7.08(1 \mathrm{H}, \mathrm{d}, J=2.0 \mathrm{~Hz}), 5.30(1 \mathrm{H}, \mathrm{s}), 2.35(3 \mathrm{H}, \mathrm{s}), 0.35(9 \mathrm{H}, \mathrm{s}) ;{ }^{13} \mathrm{C} \mathrm{NMR}\left(\mathrm{CDCl}_{3}, 125 \mathrm{MHz}\right) \delta$ 154.79, 137.35, 135.21, 131.85, 129.38, 129.24, 129.17, 127.80, 126.88, 125.54, 20.52, -0.90; IR (film) v 3558, 3080, 3058, 3029, 2953, 2898, 2734, 1954, 1893, 1814, 1605, 1588, 1495, 1455, $1424,1318,1224,1193,1164,1072,1028,913,838,772,737,703,626 \mathrm{~cm}^{-1}$. 


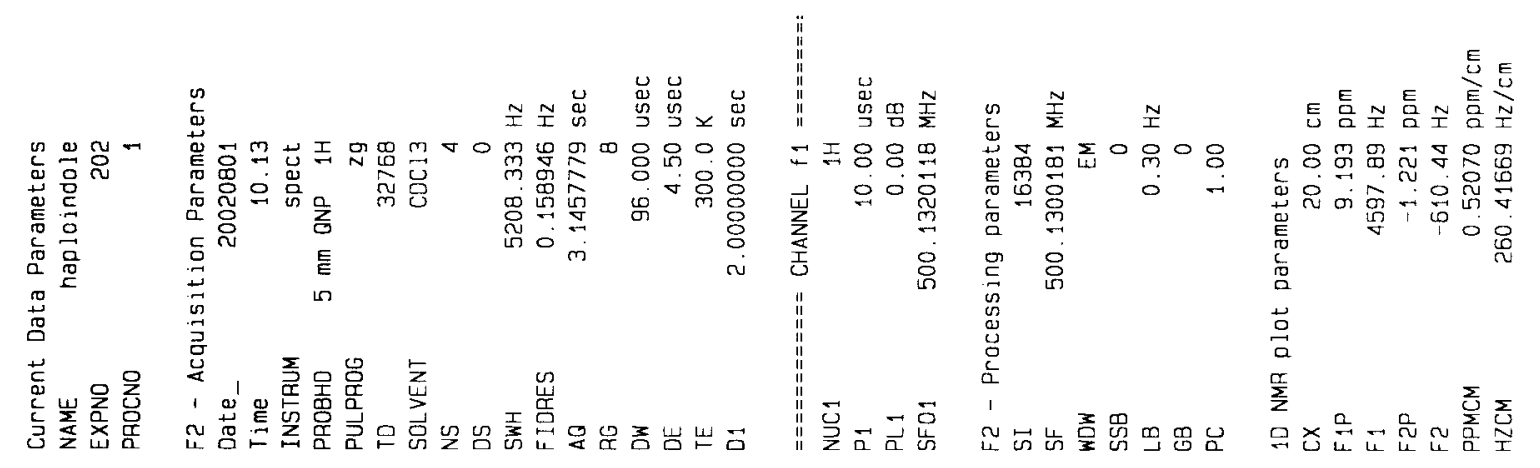

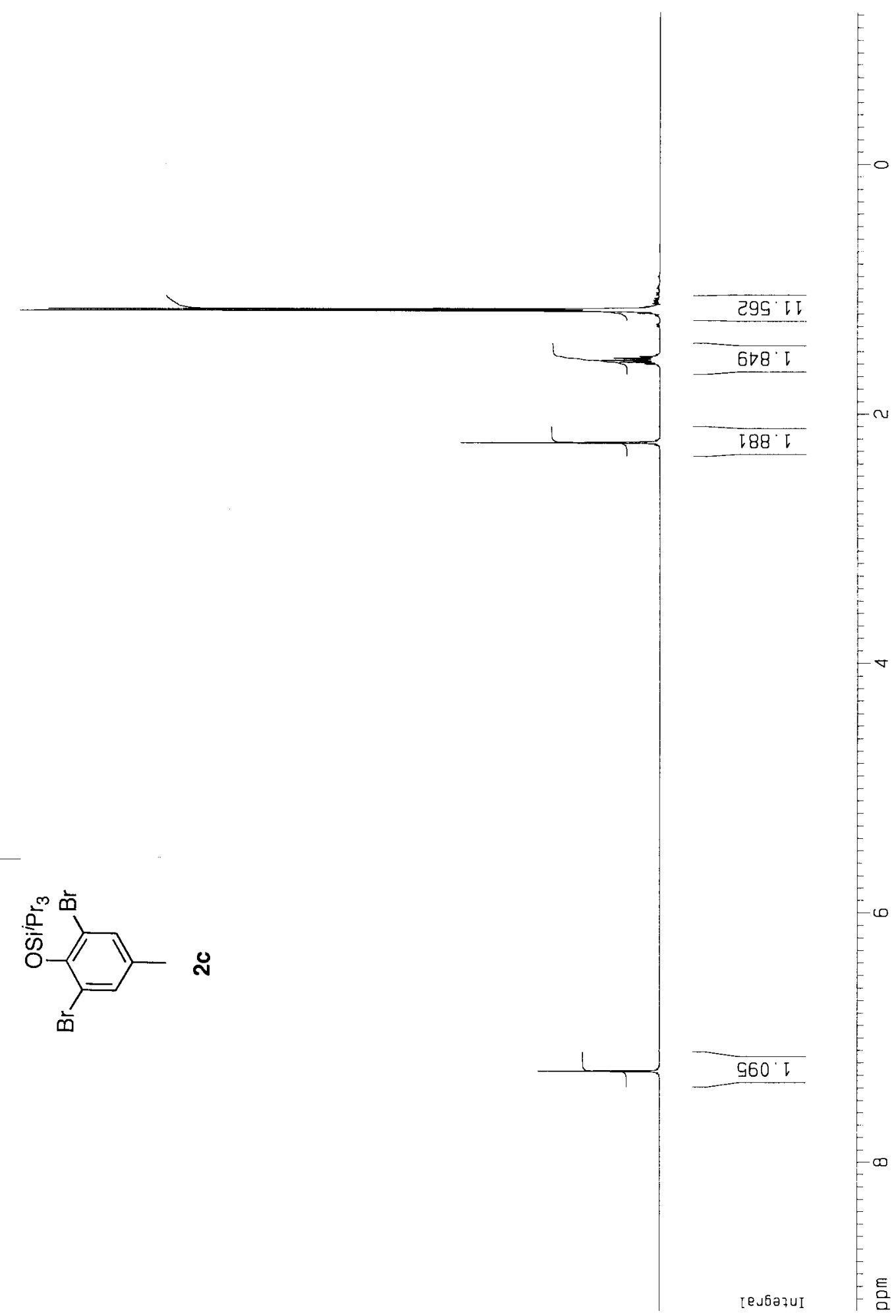




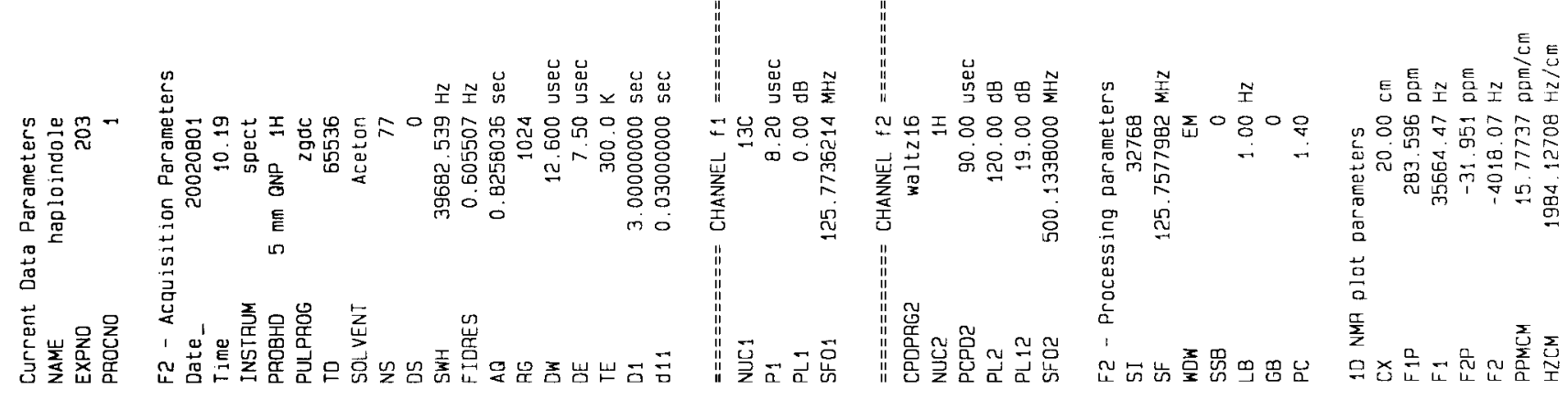

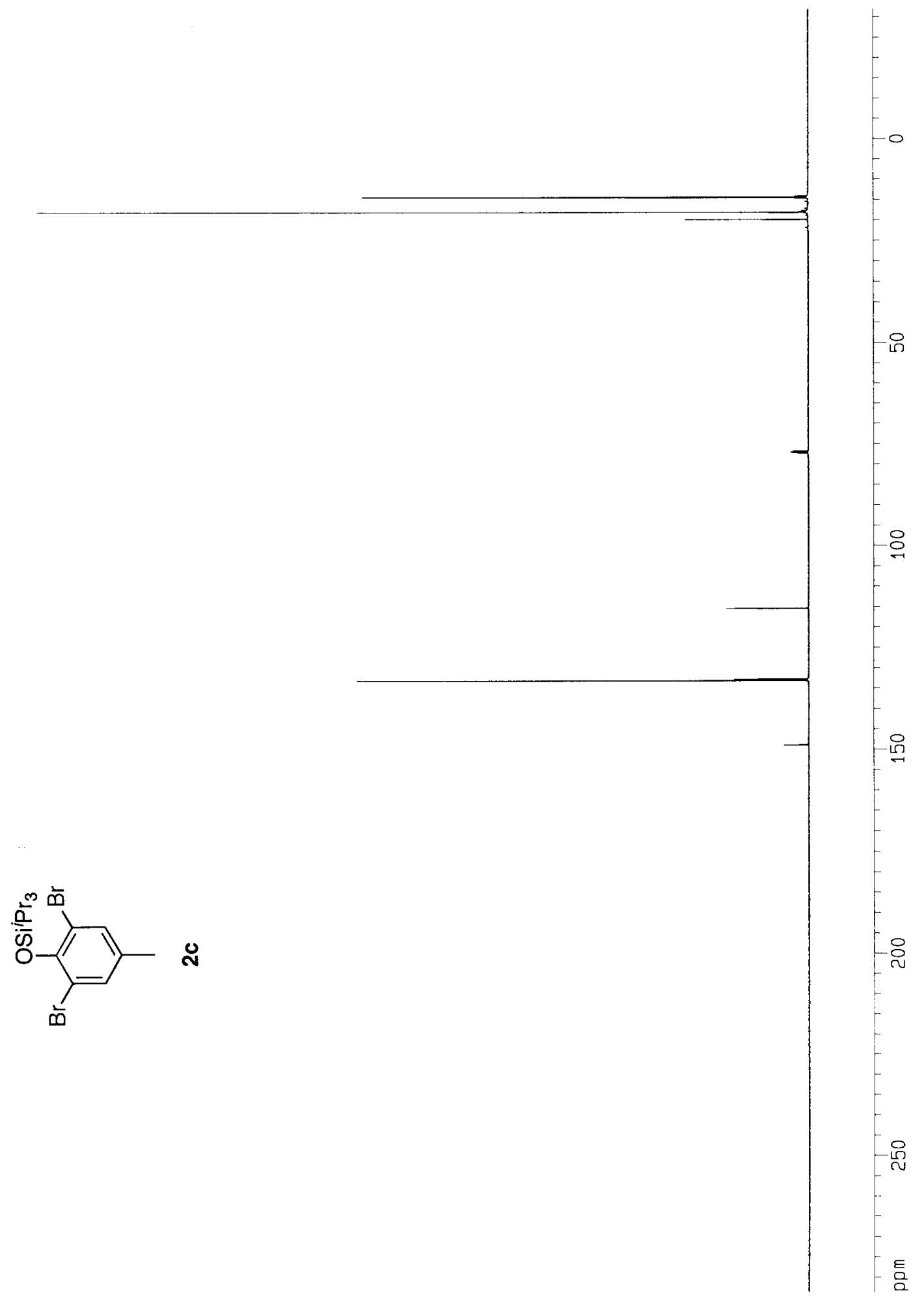




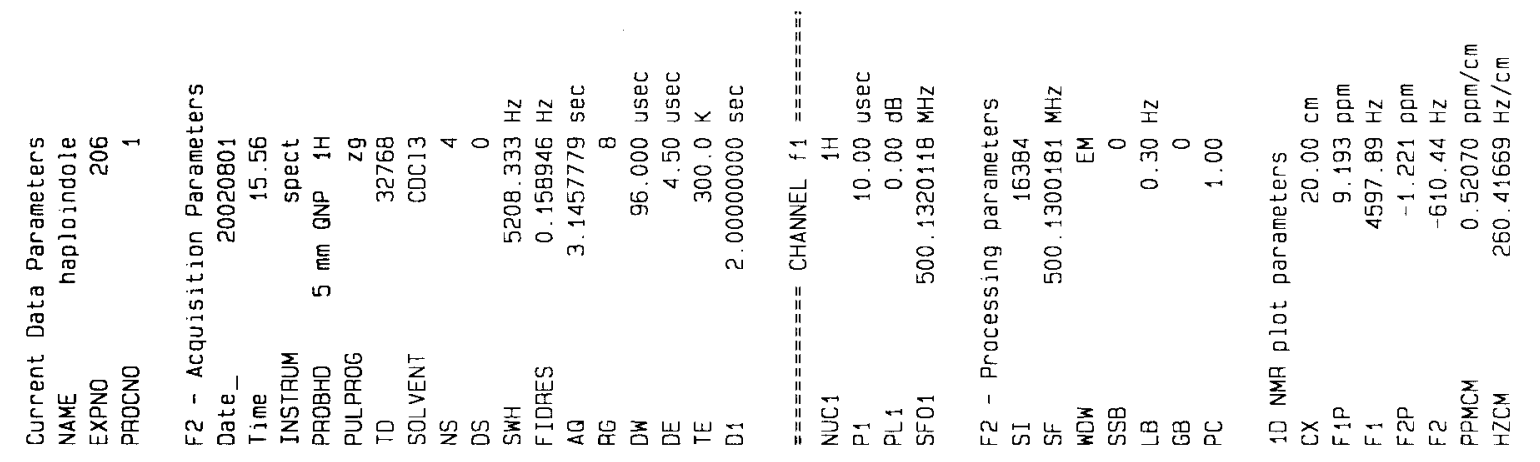

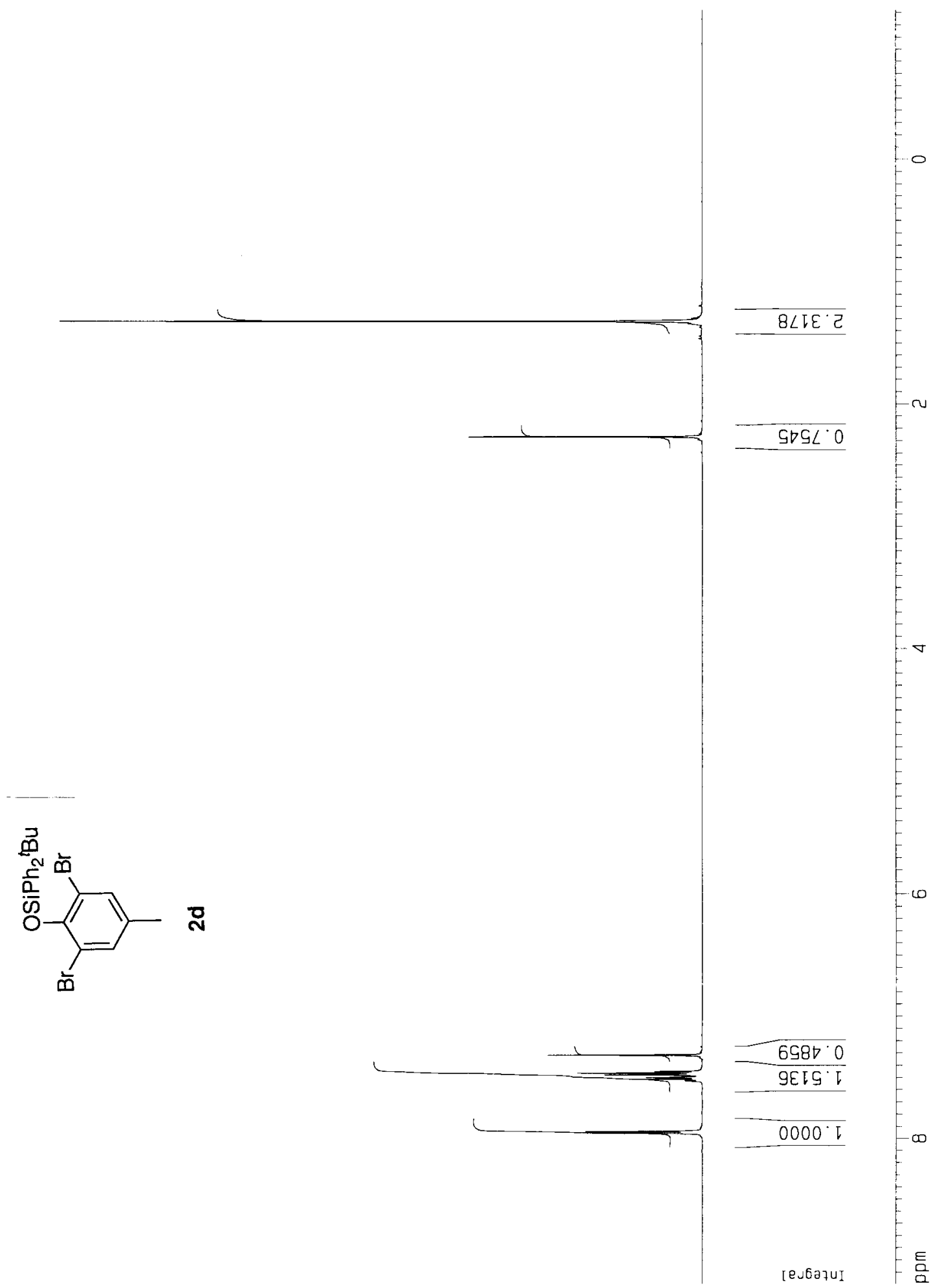




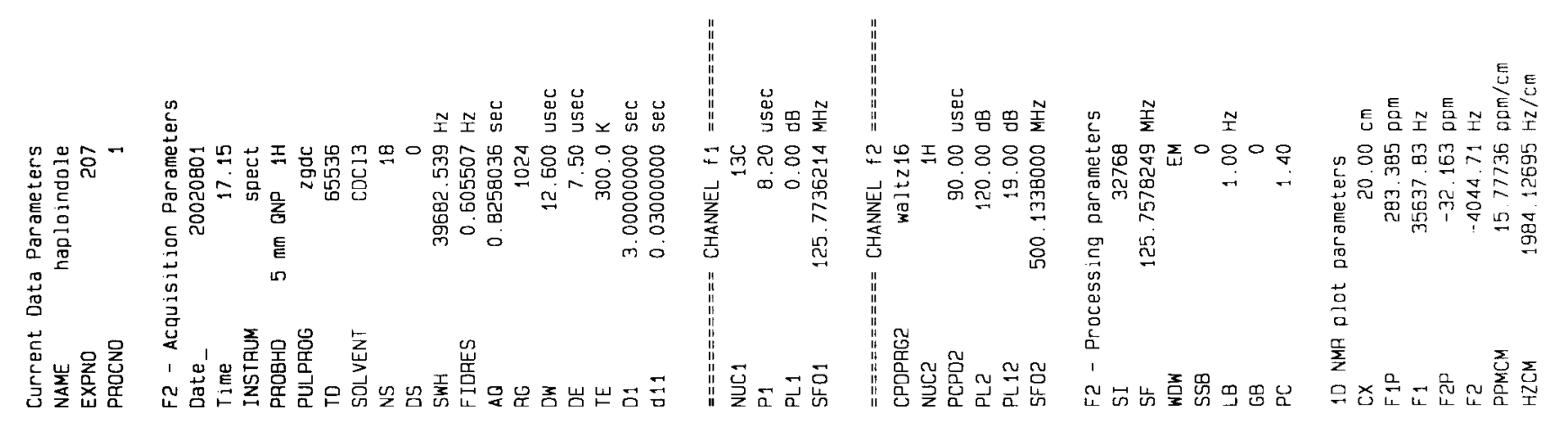

$09 \angle 6 I$

$\angle G E O Z \square$

$959^{\circ} 92$
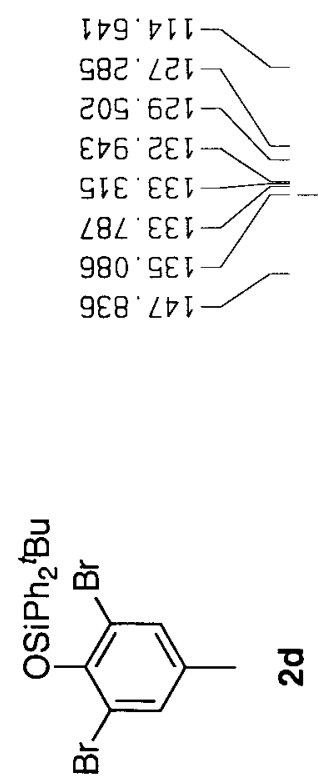

wdd 

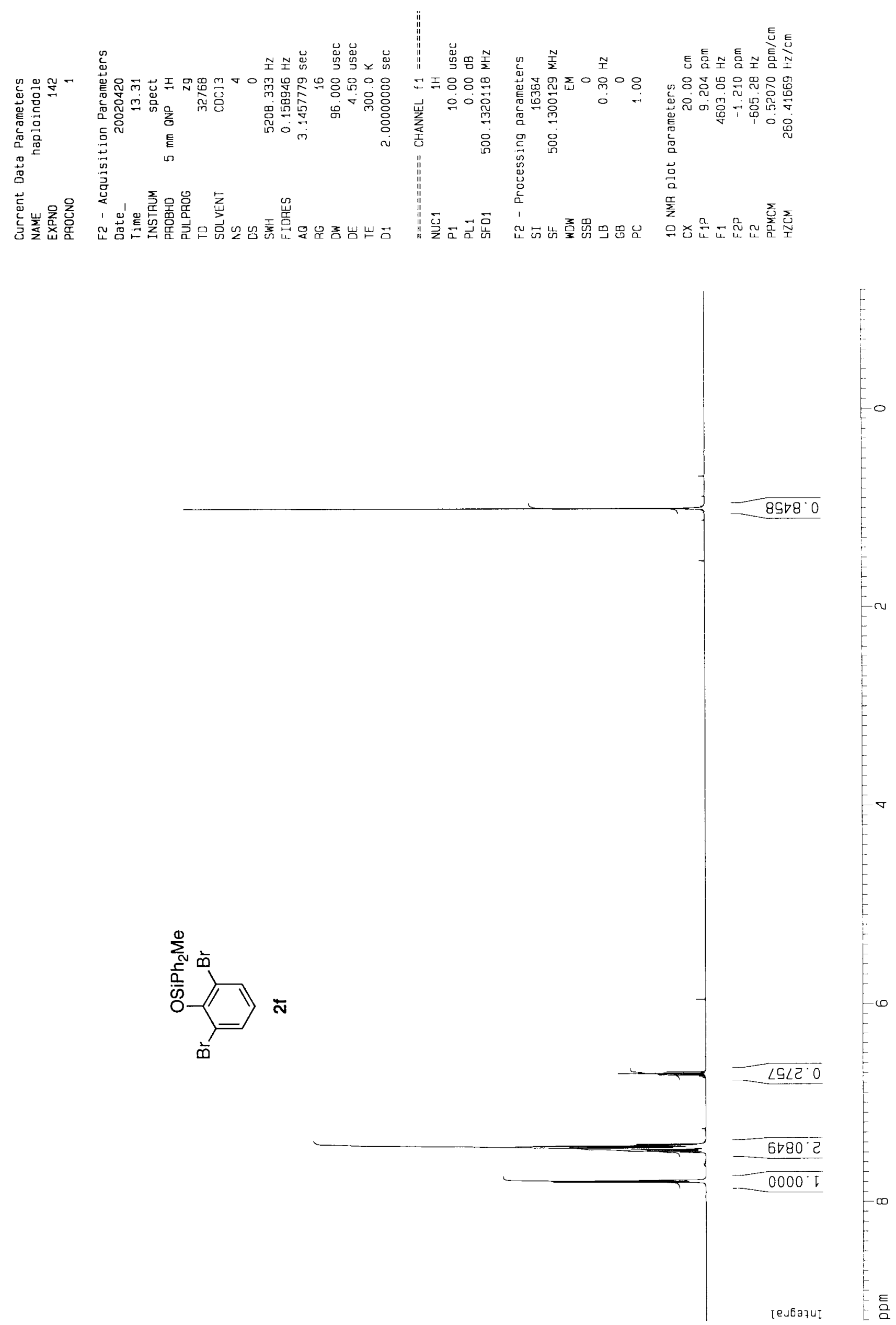


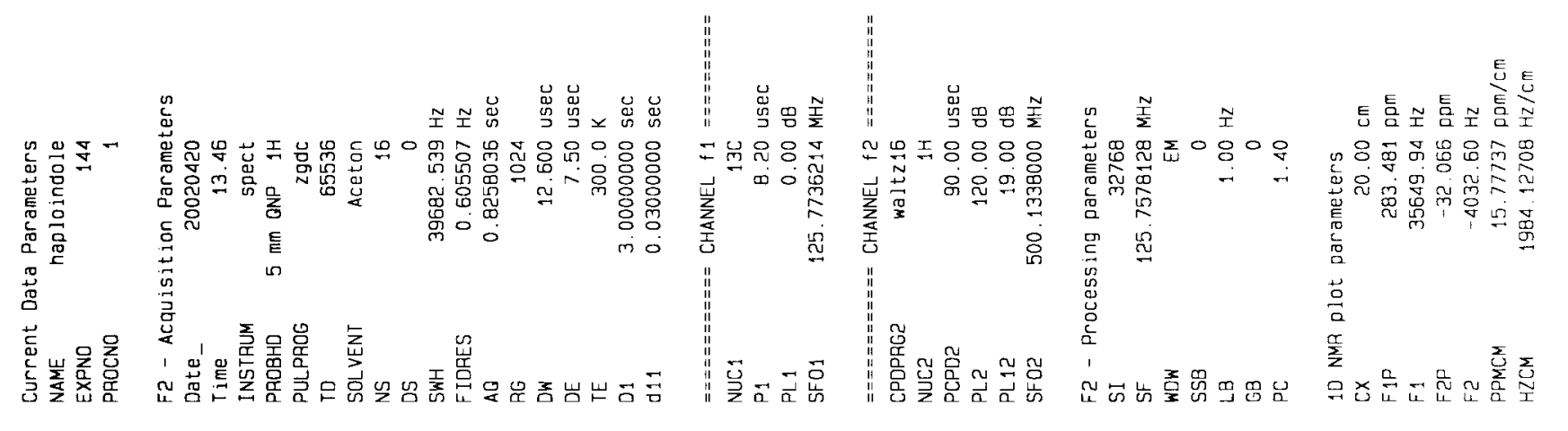

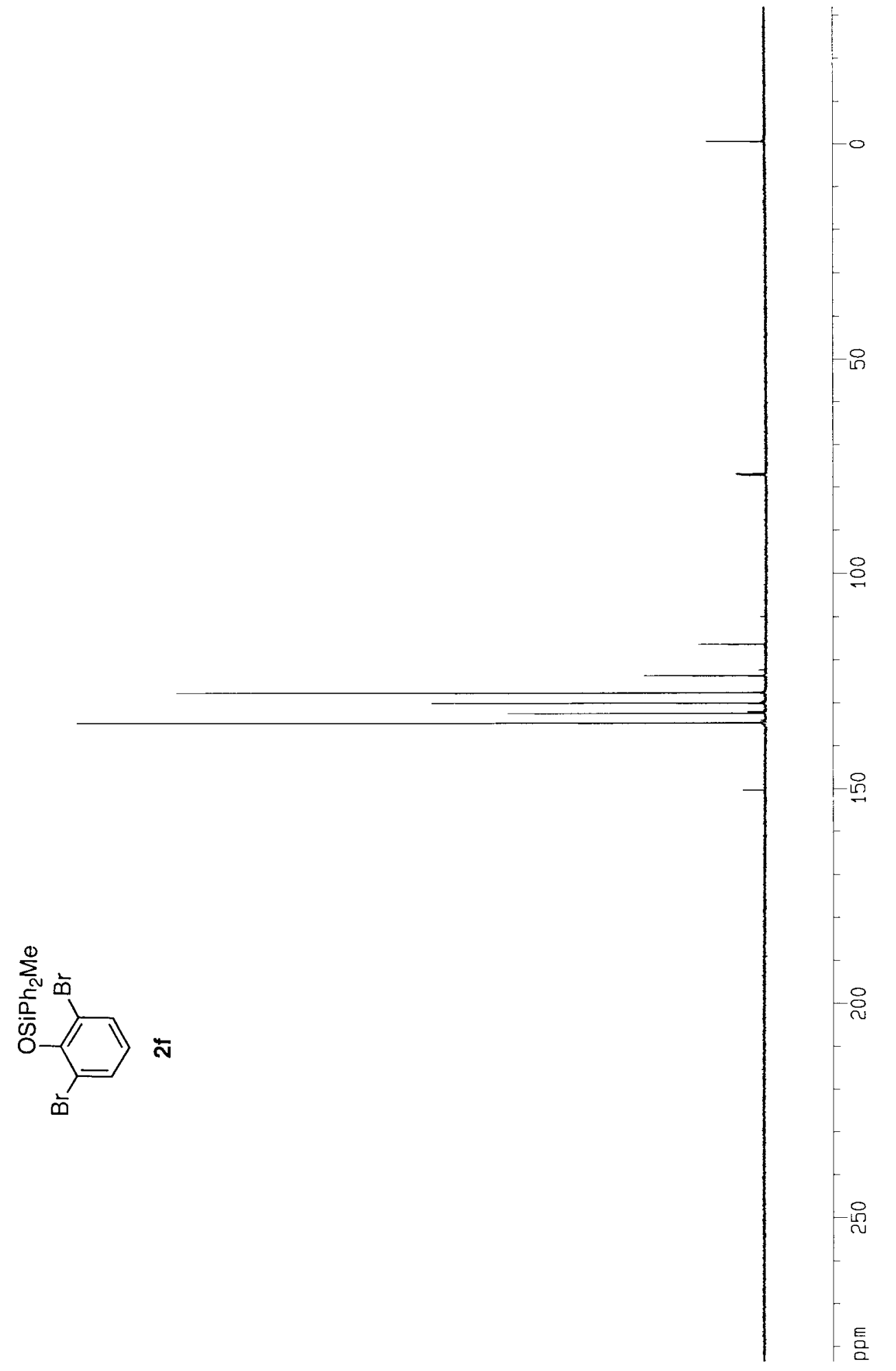




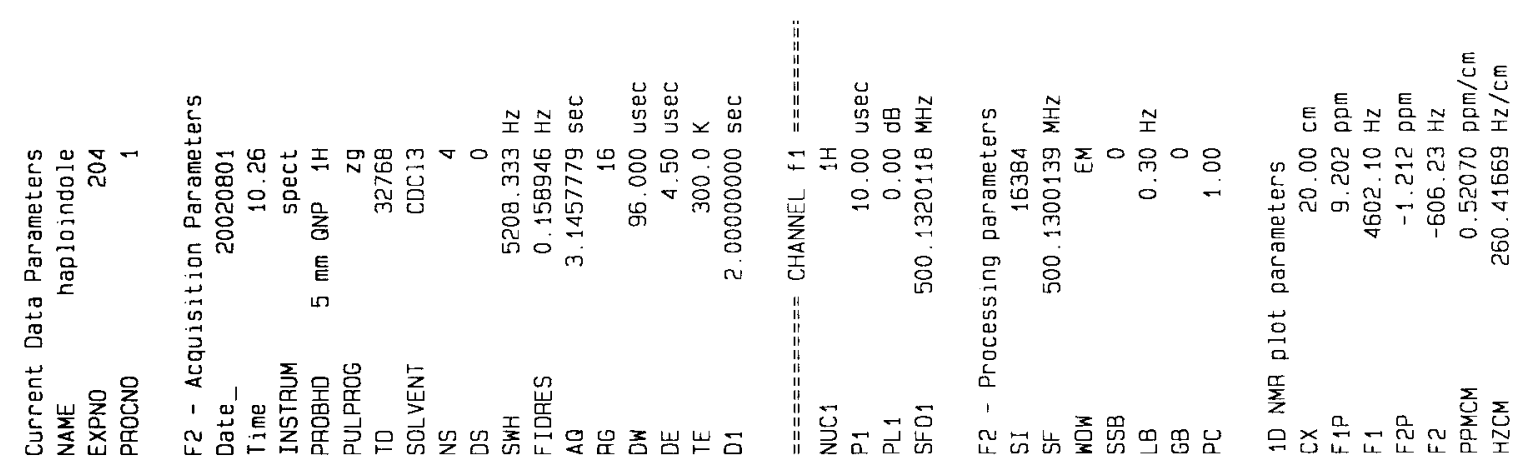

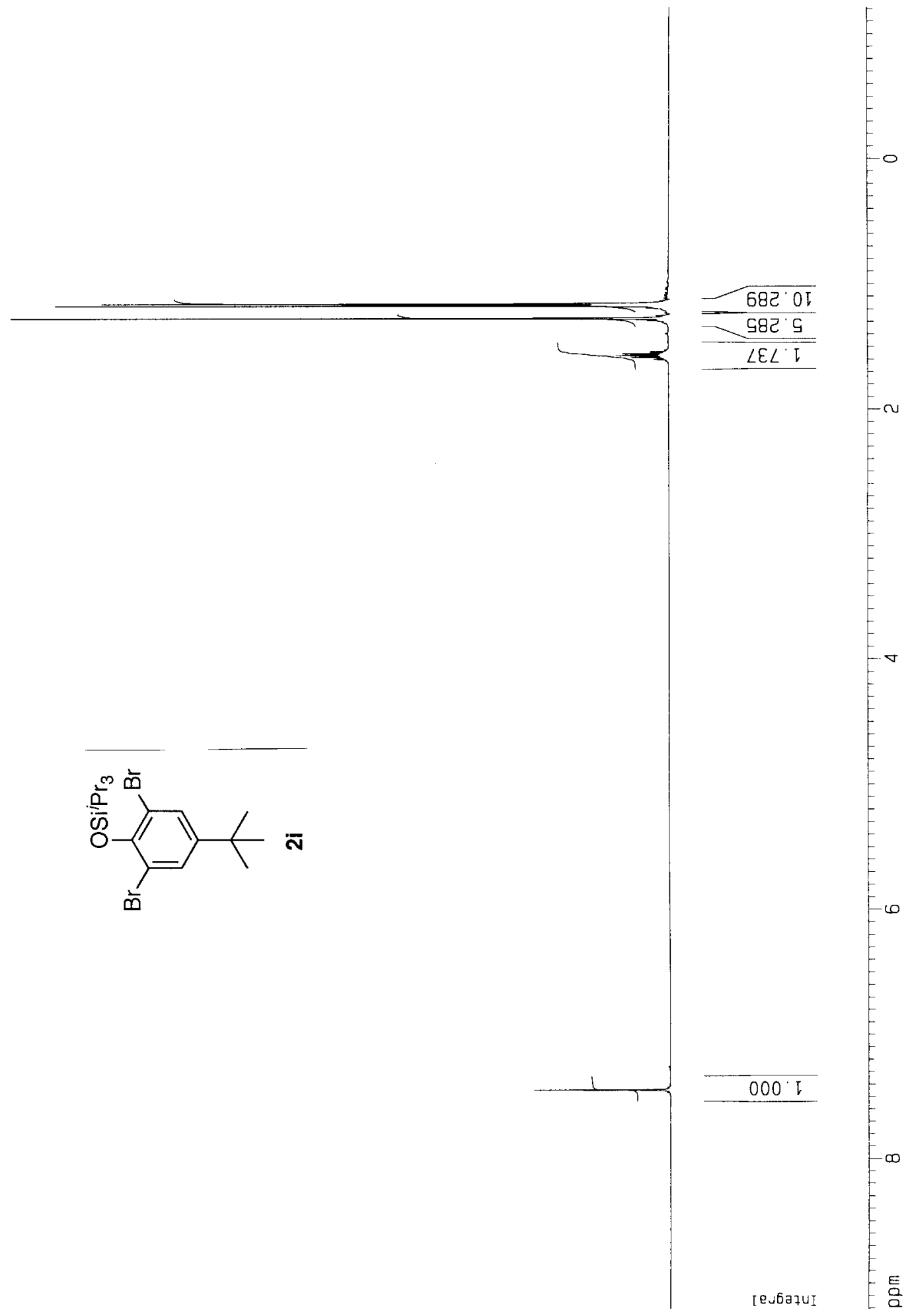



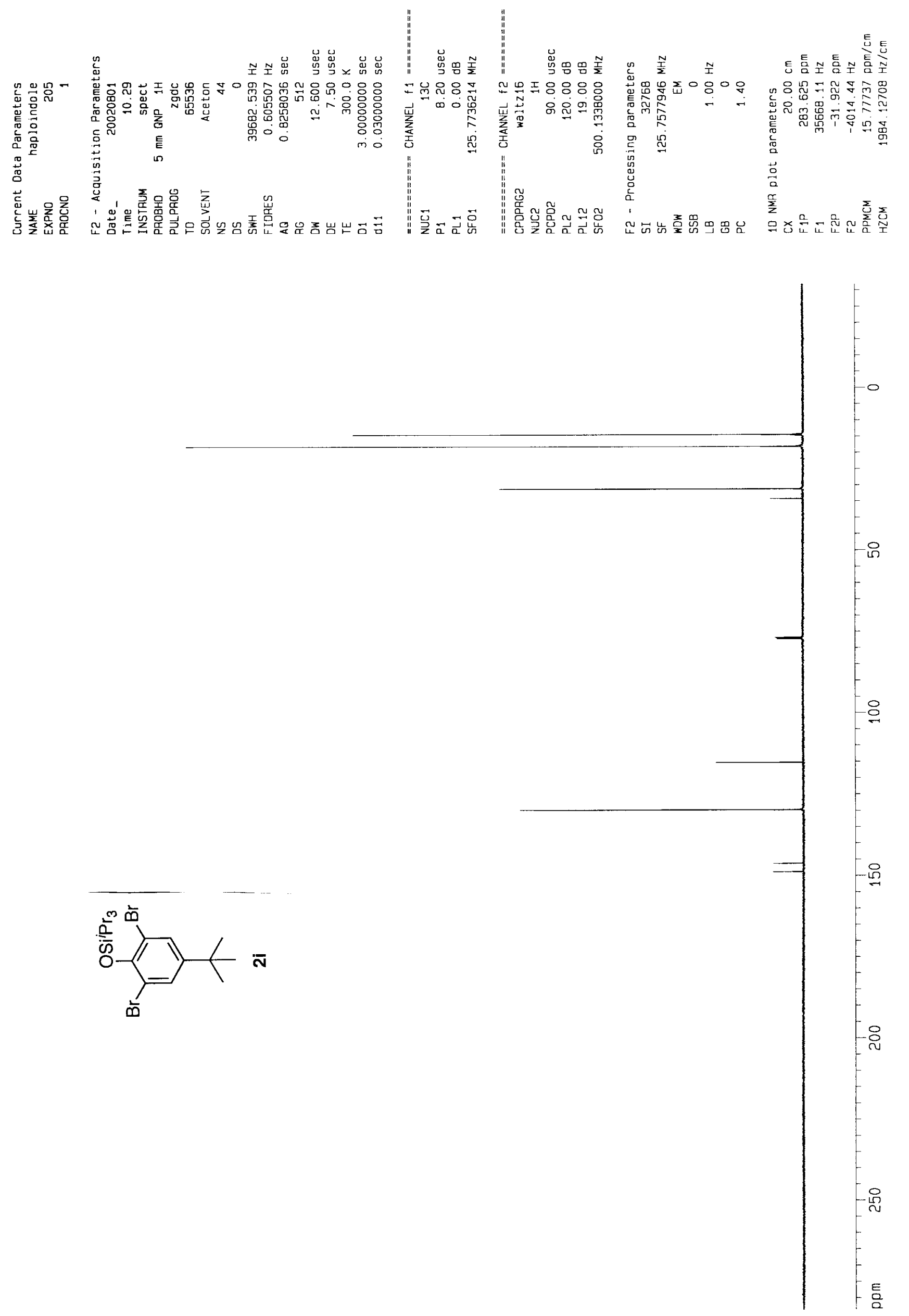

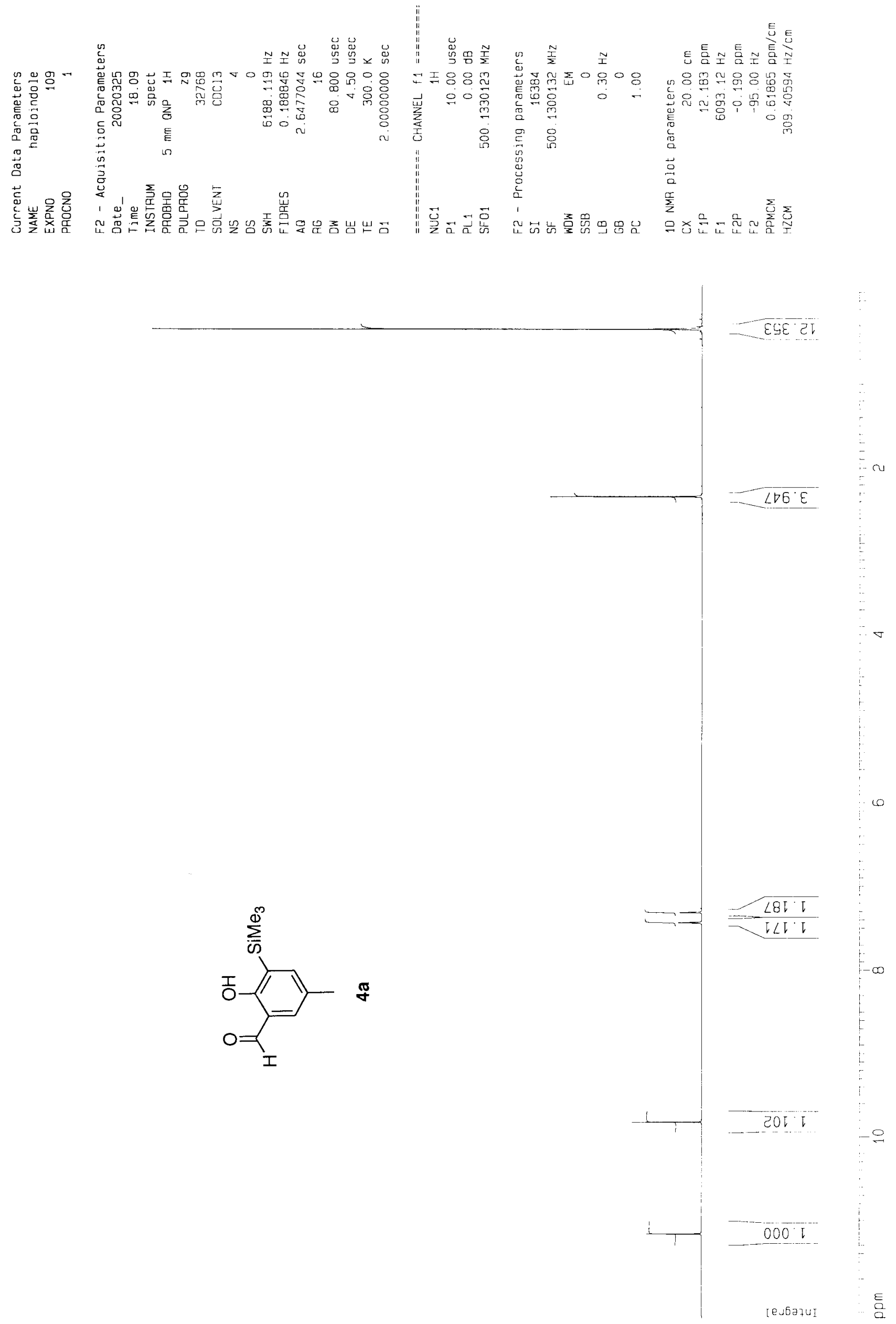


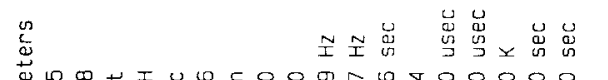
$\begin{array}{lll} & \\ & & \\ 0\end{array}$ $\stackrel{E}{n}$

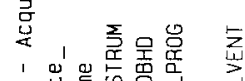

\section{压}

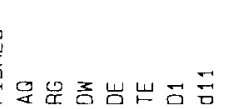

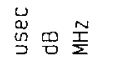

造뭄욤

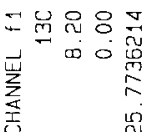

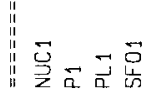

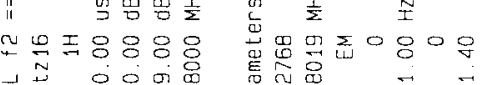

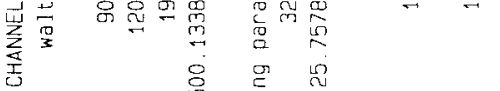

華地

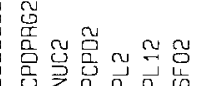

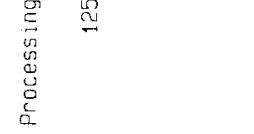

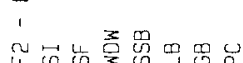

E

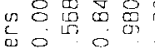

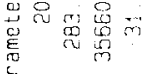
흠 荅 旁

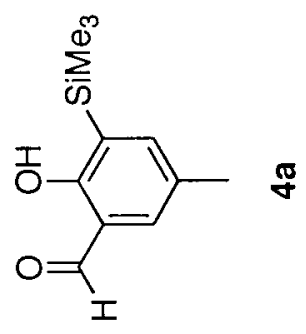



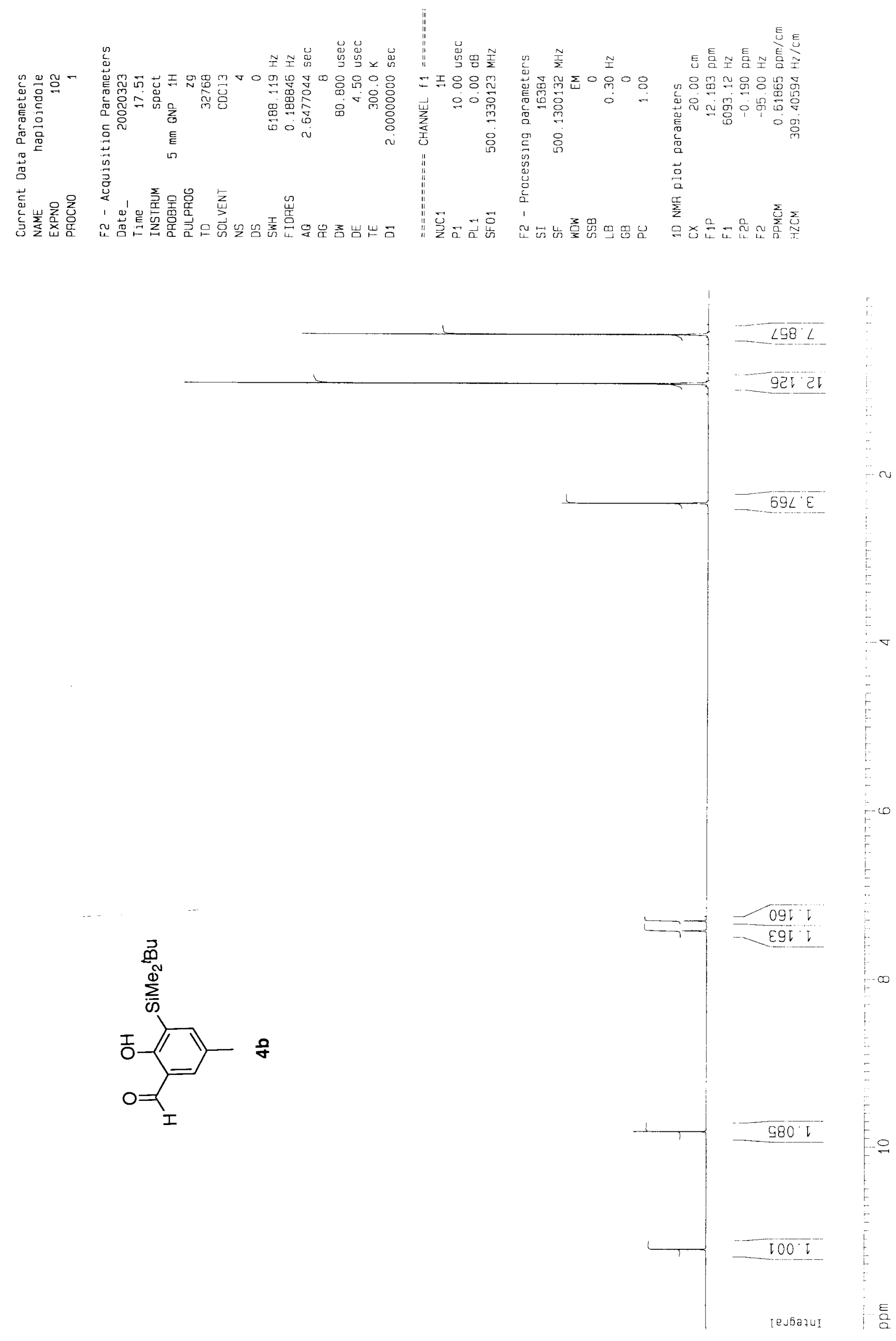


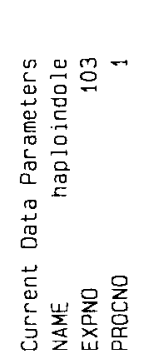

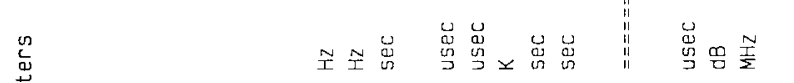

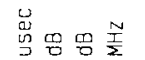

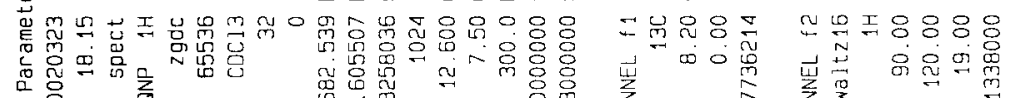

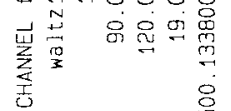

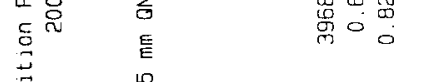

nio

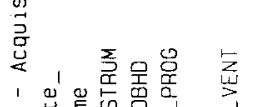

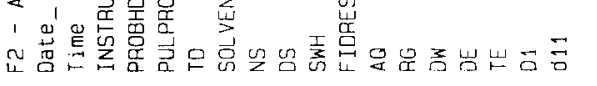

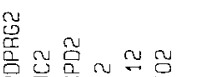

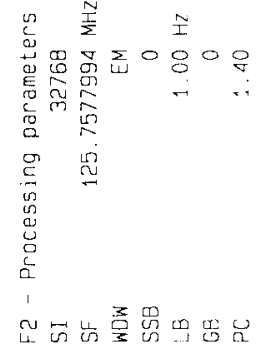

튼

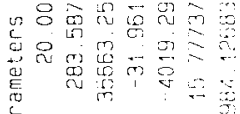

离

음

雾
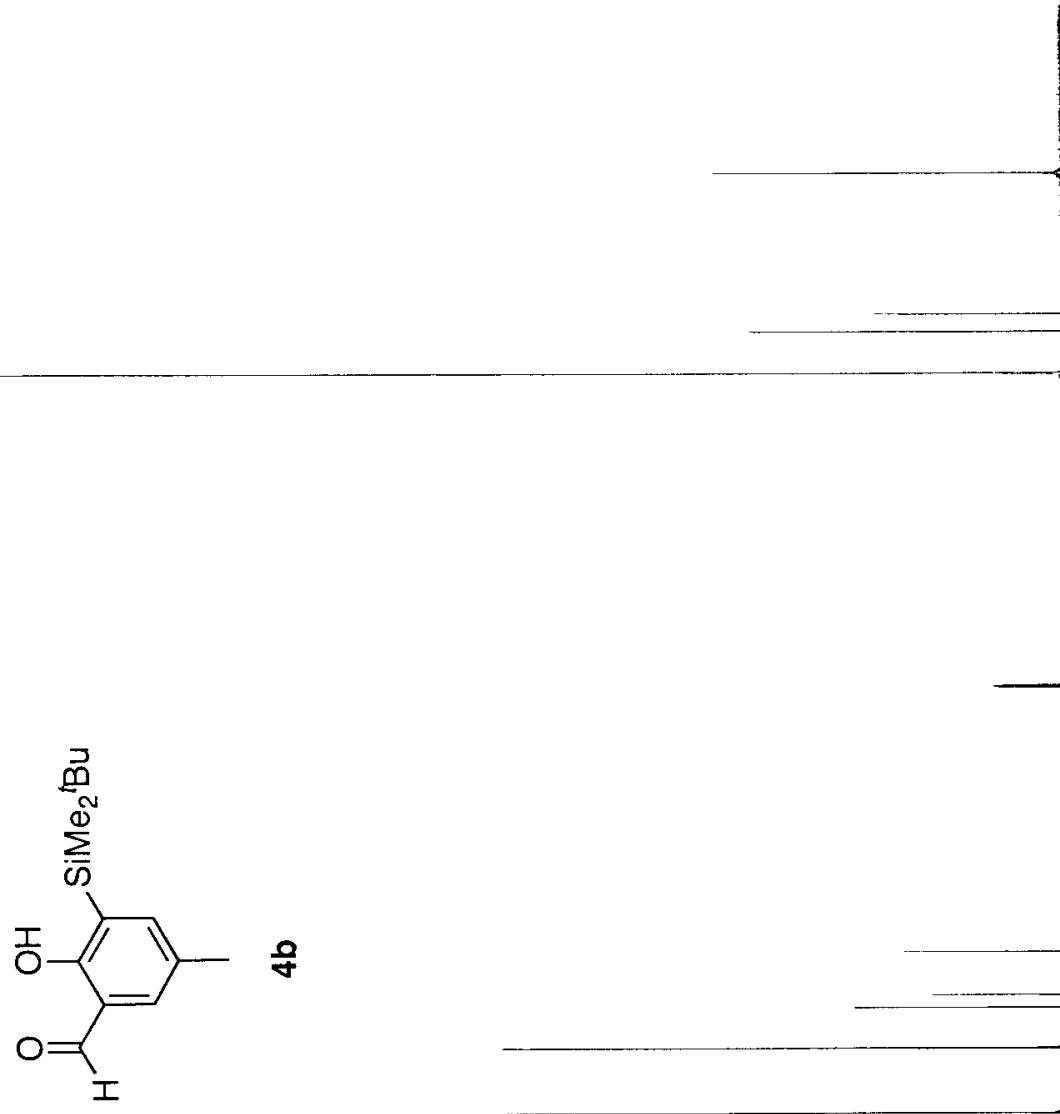


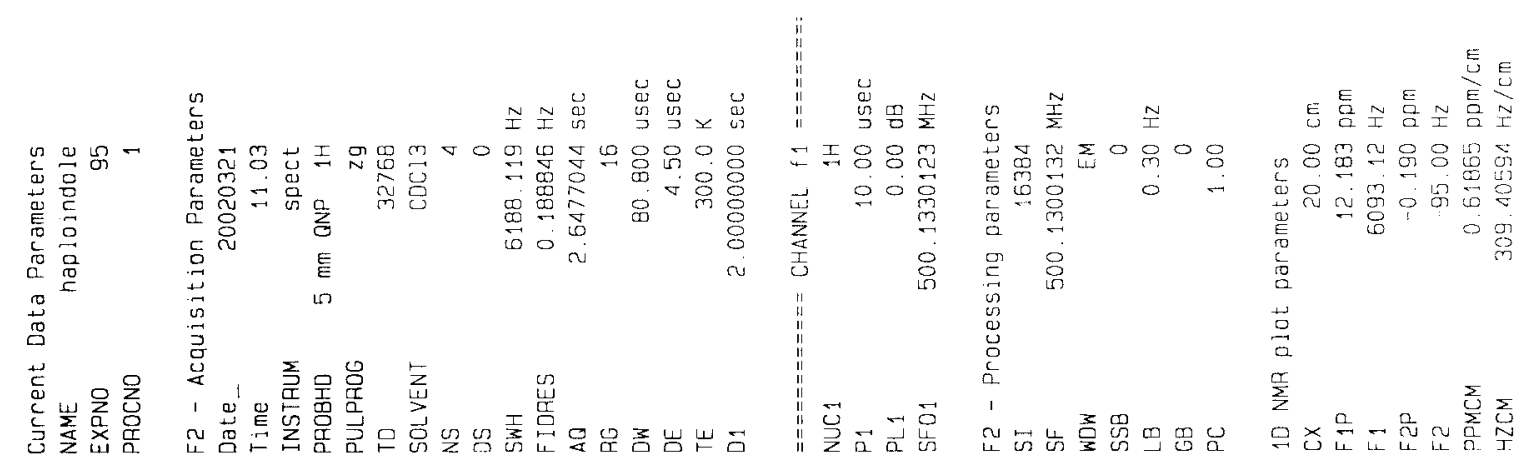

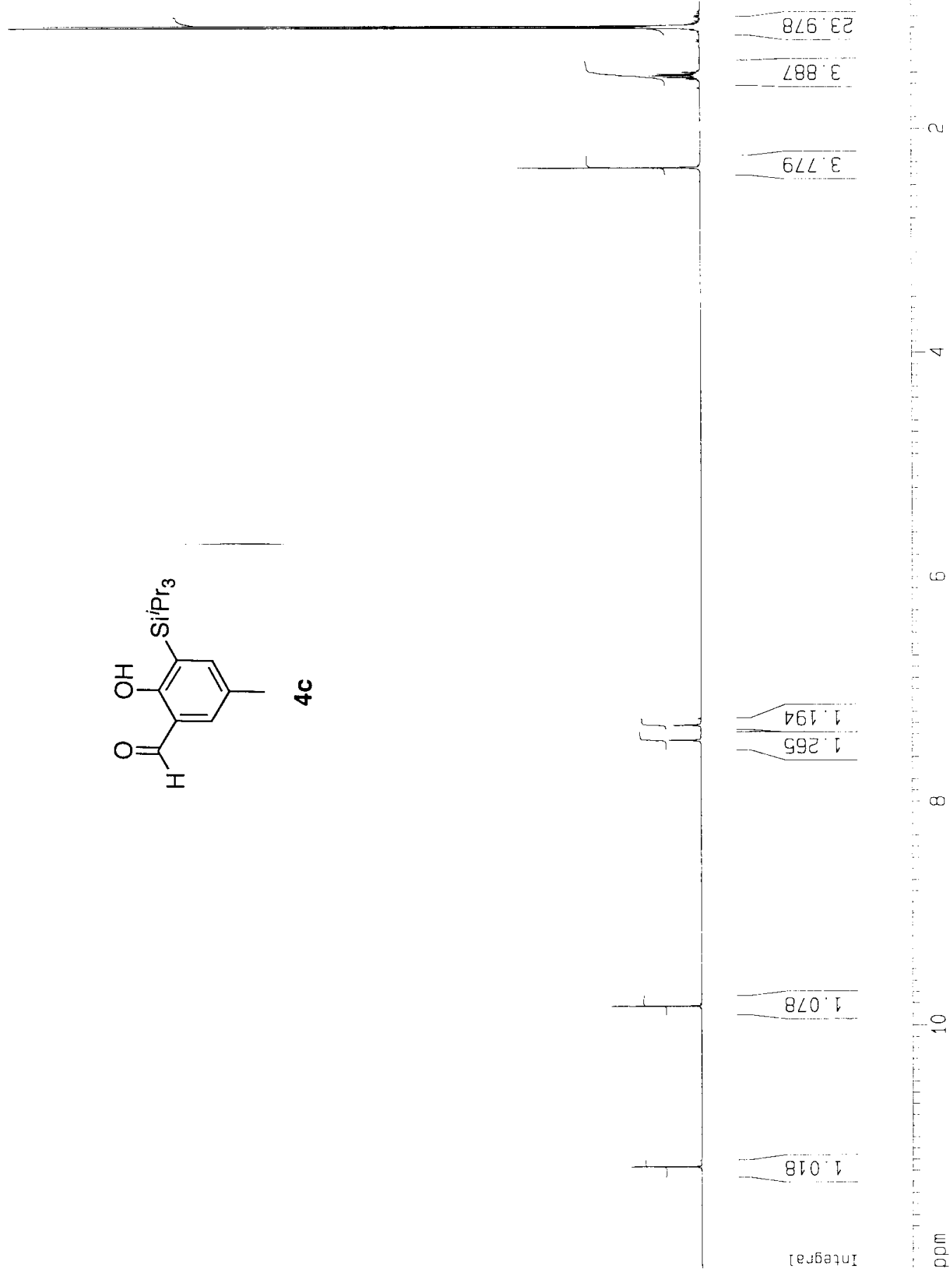




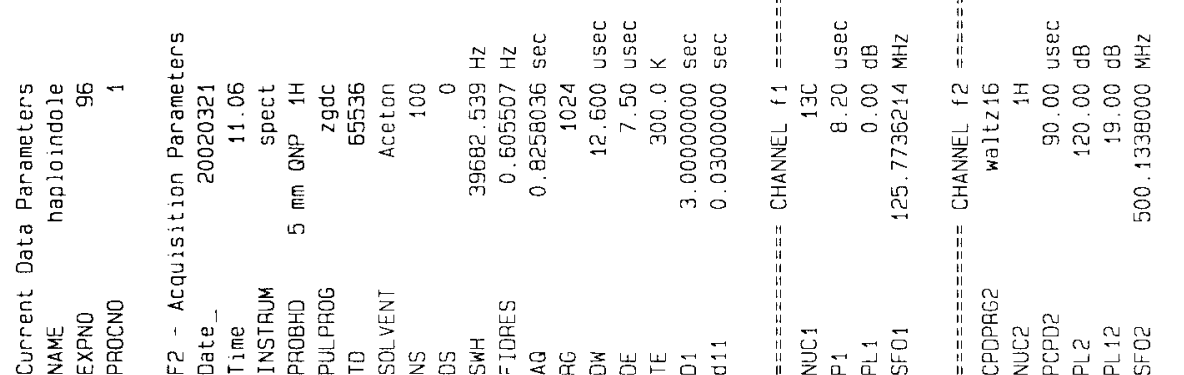

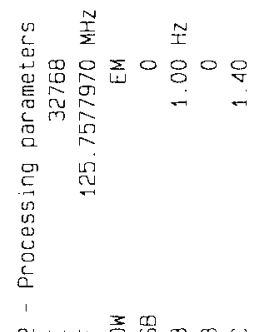

트음도을

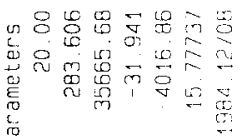

染

蒿

雾

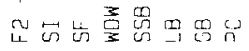

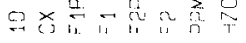

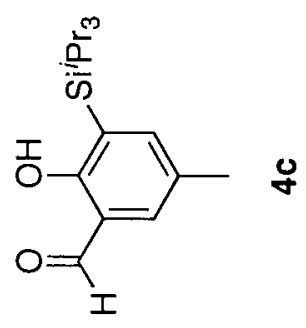



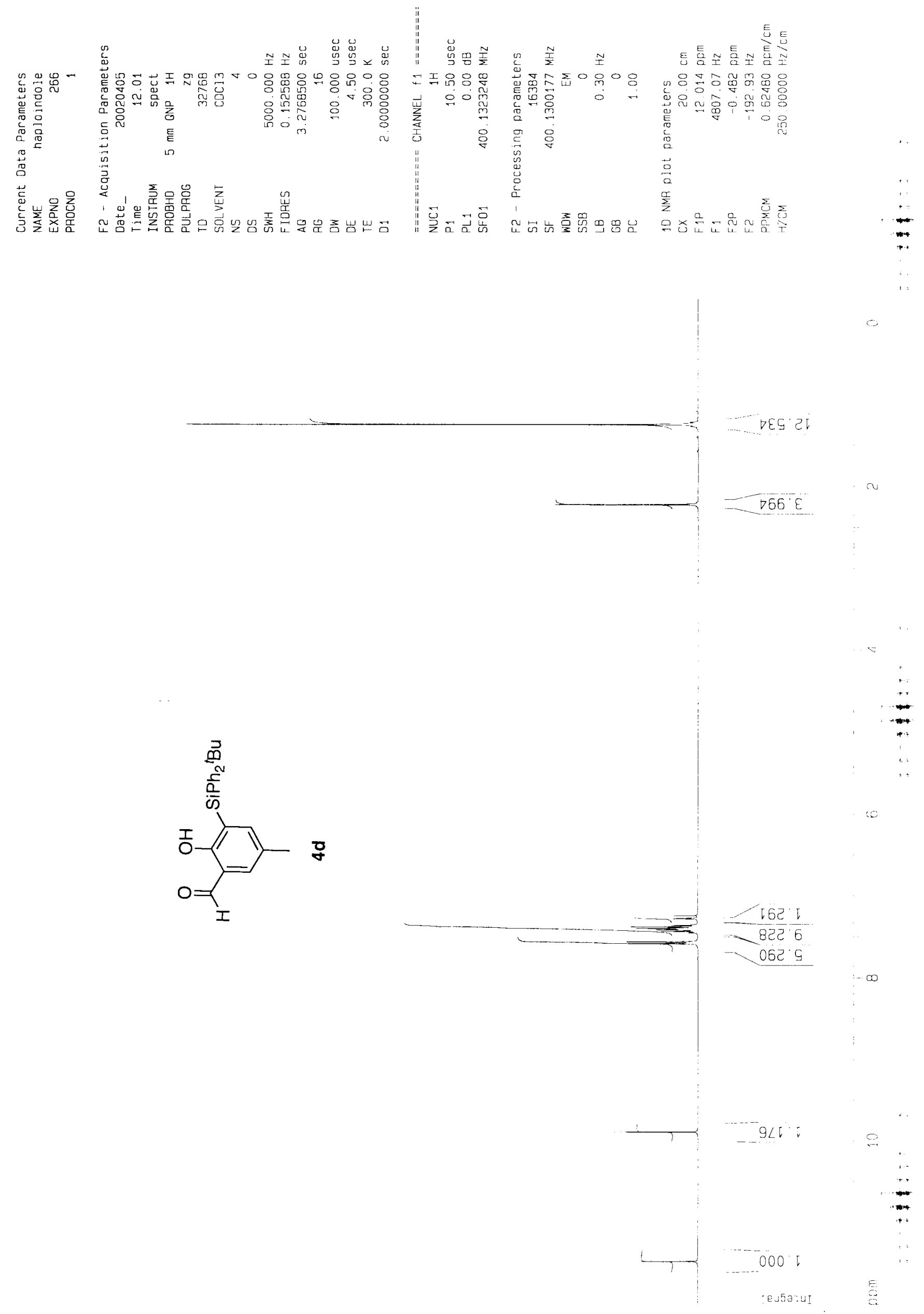


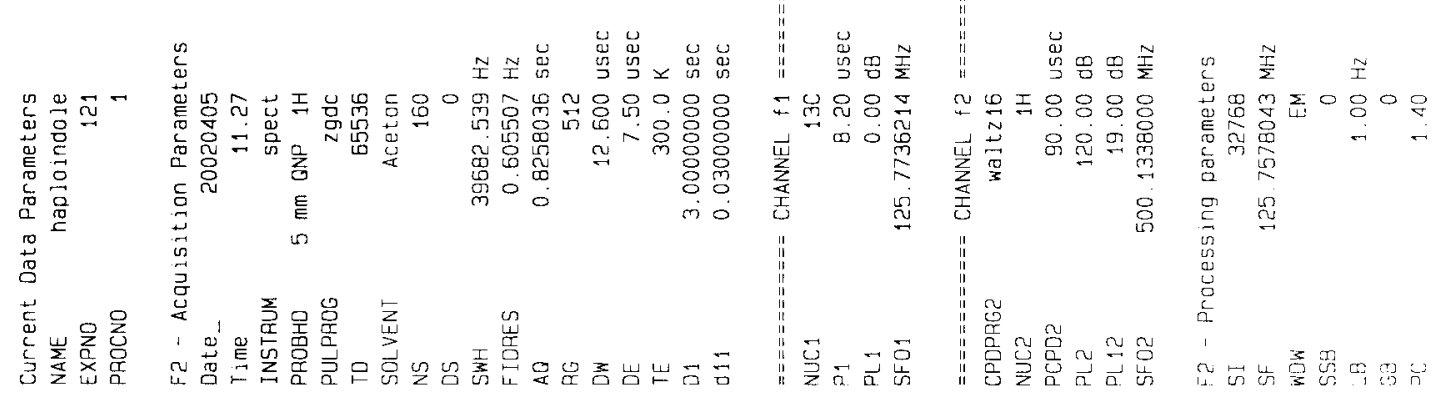
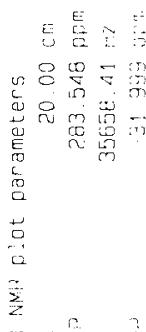
号

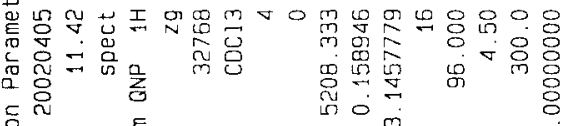

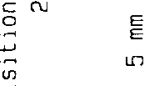

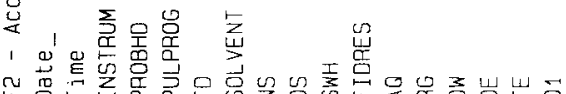

峁

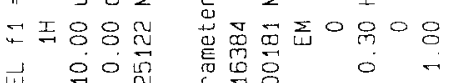

站

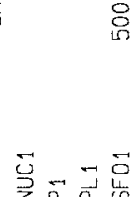

든

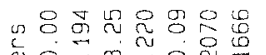

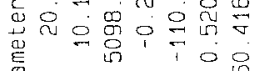
c 믐 旁
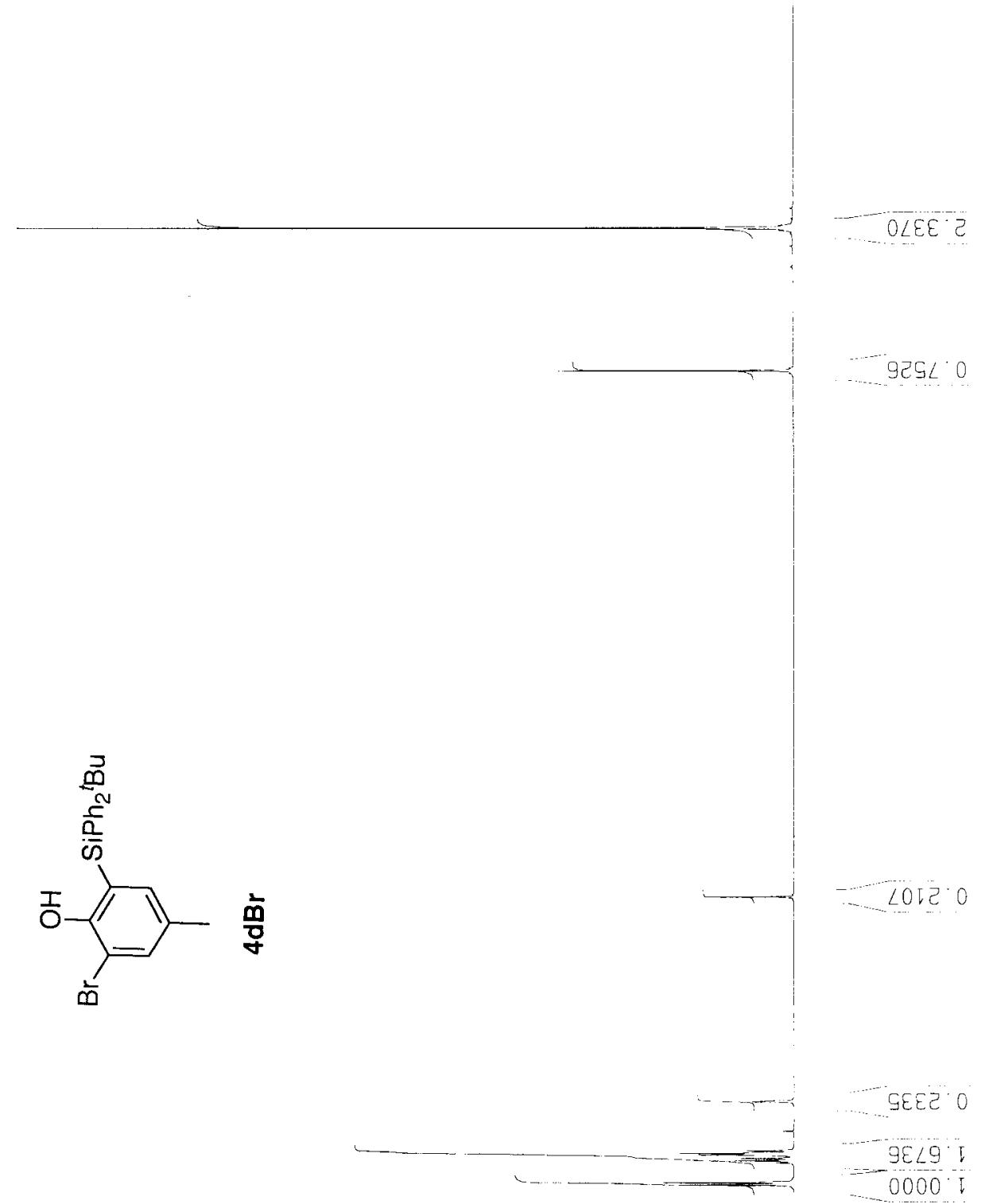


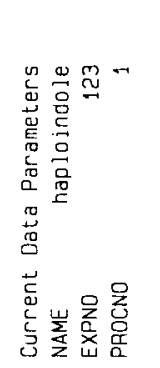

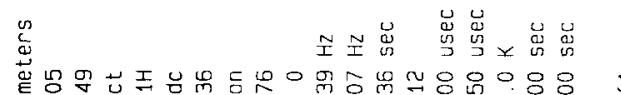

峜田

崖

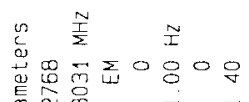

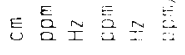

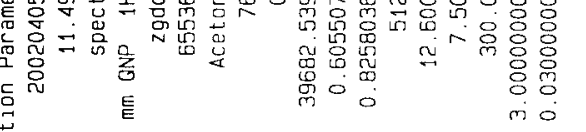

营

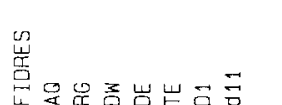

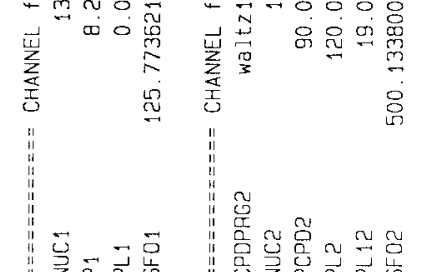

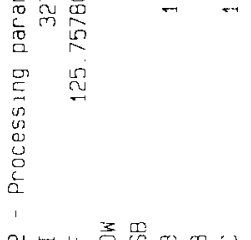

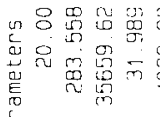

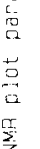

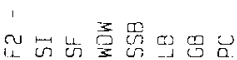
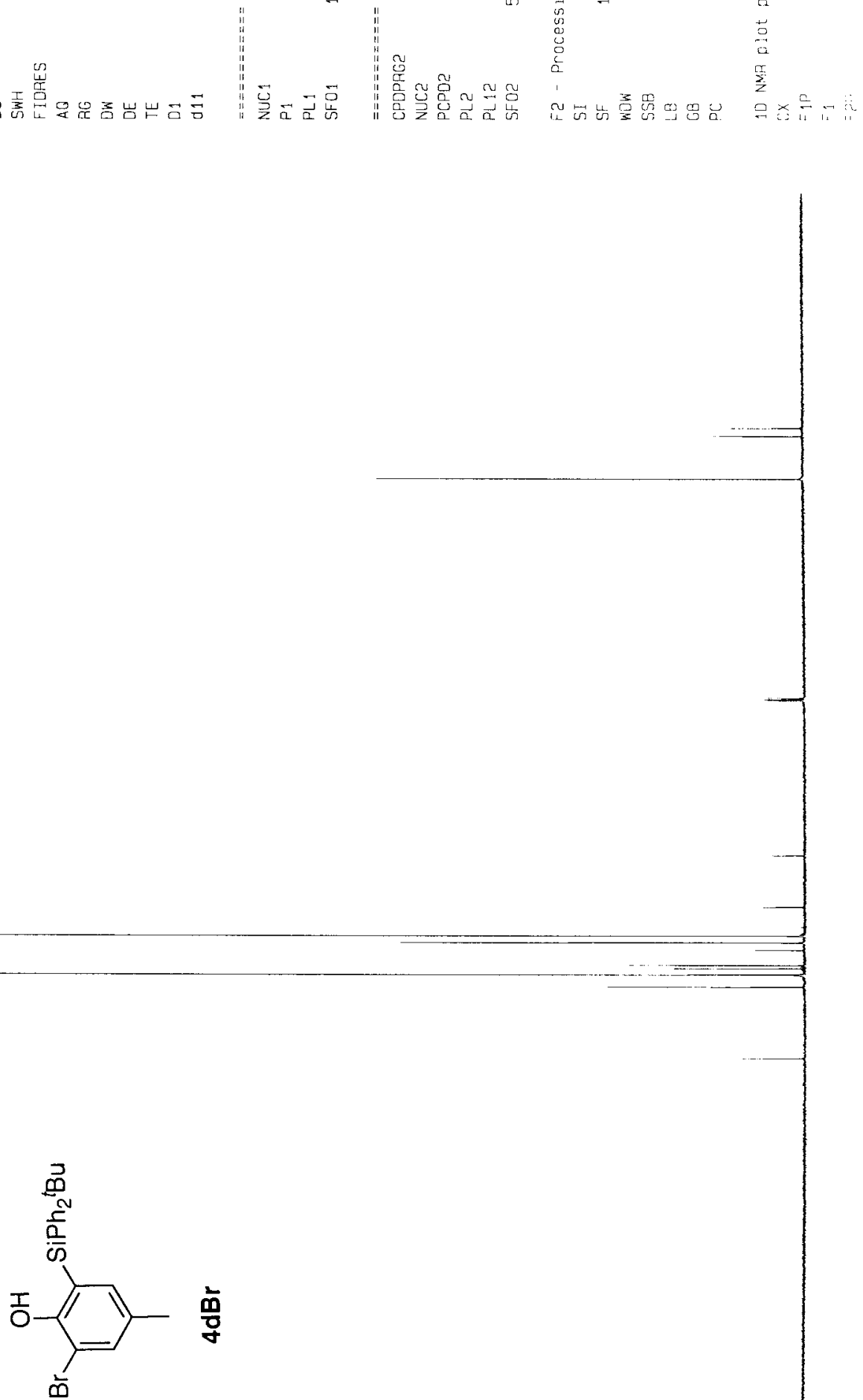


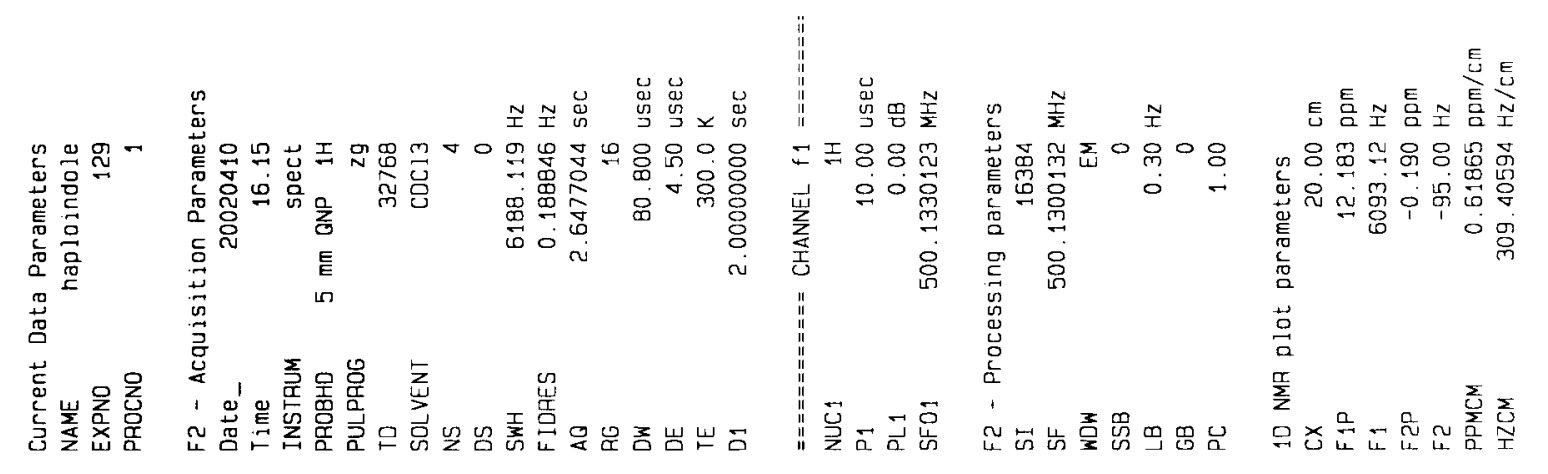

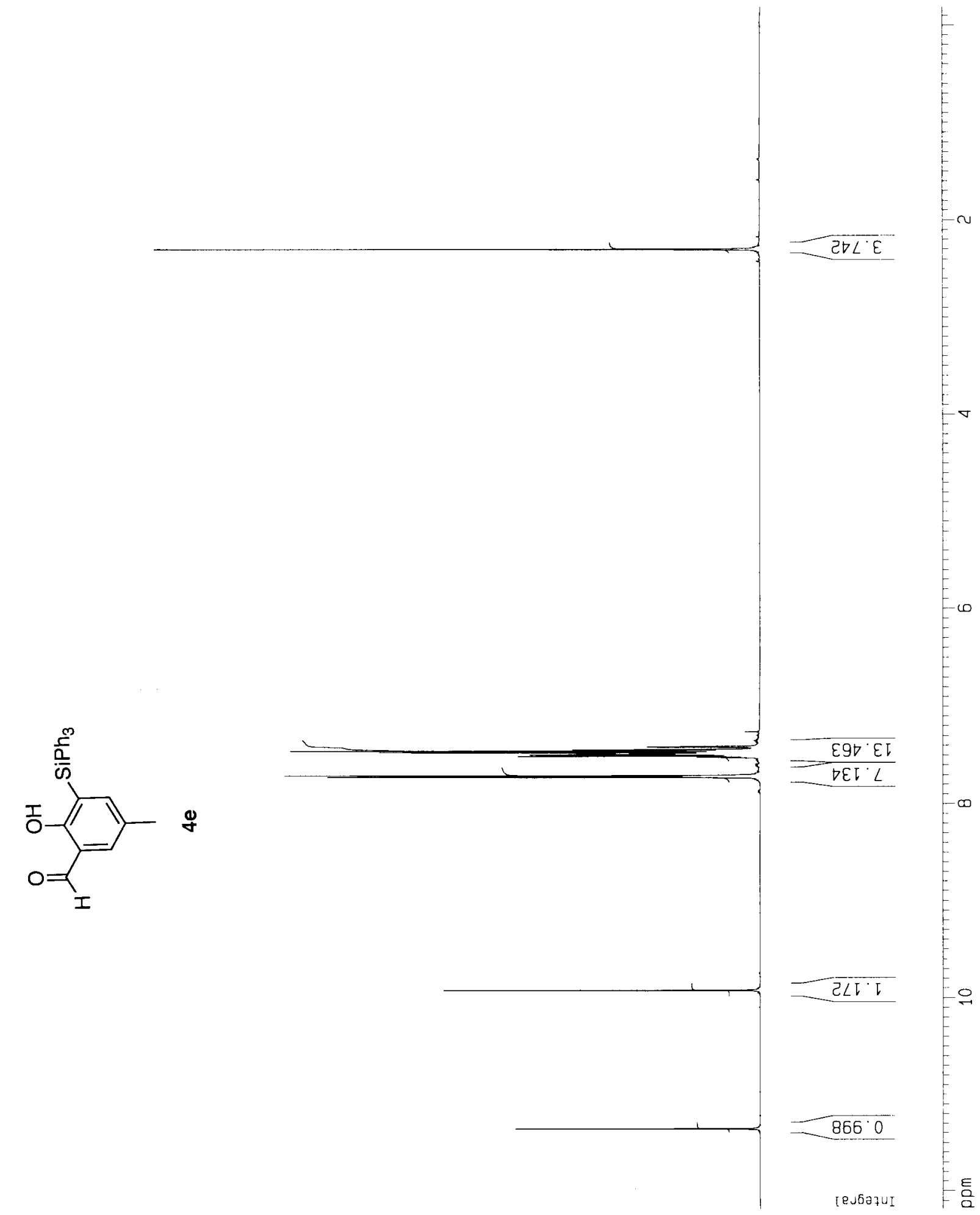




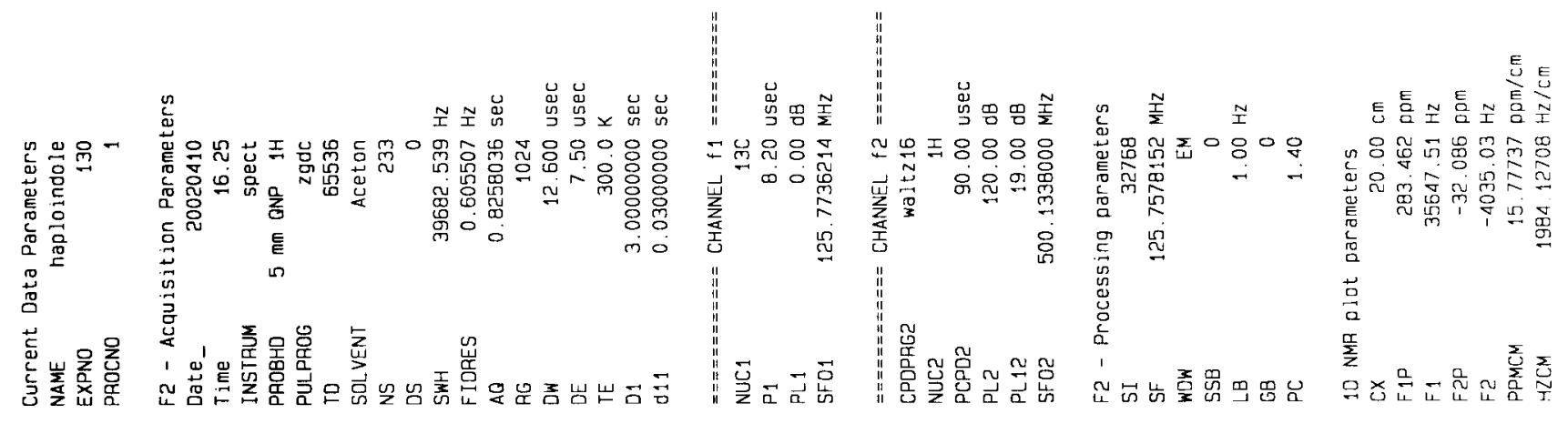

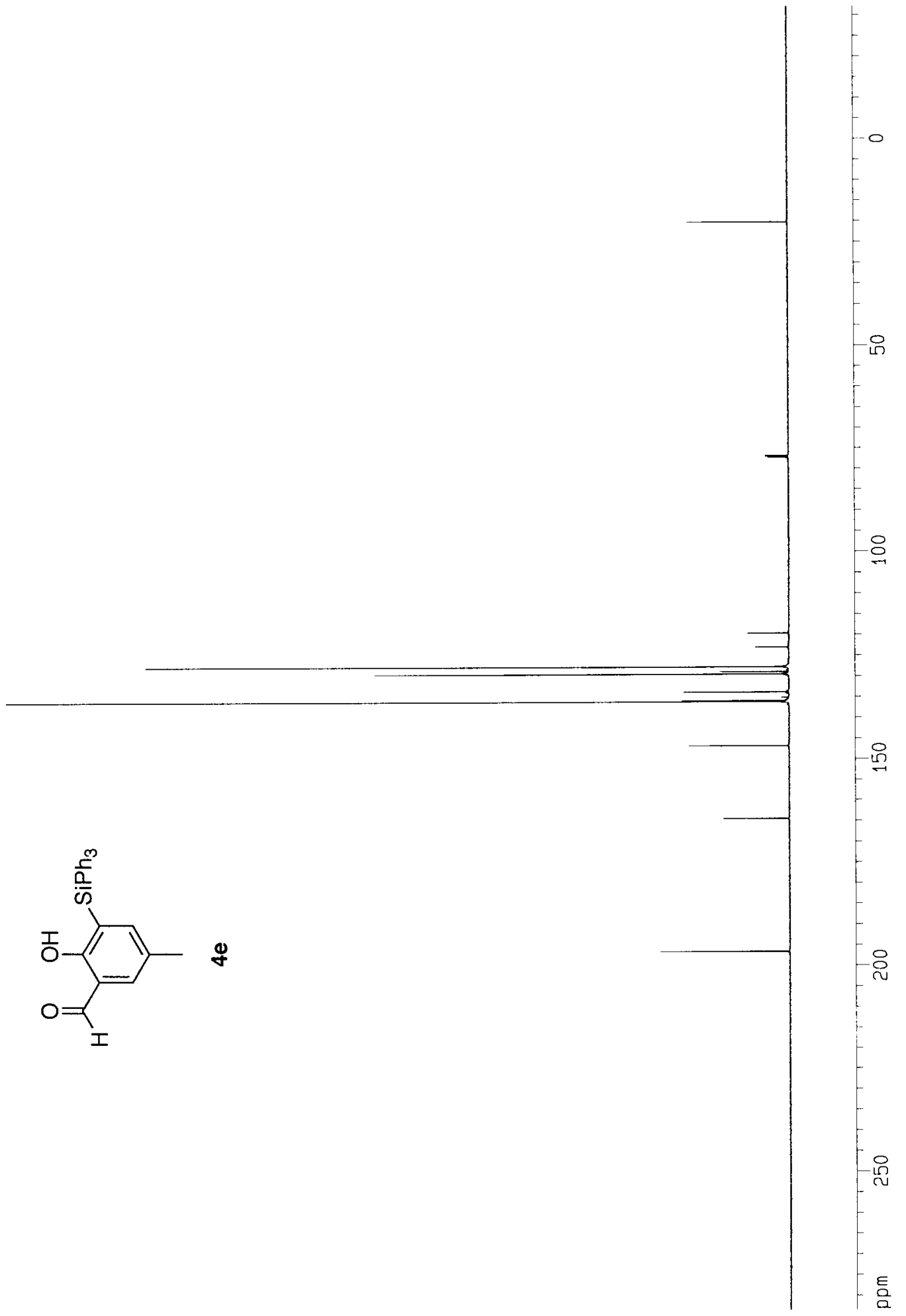




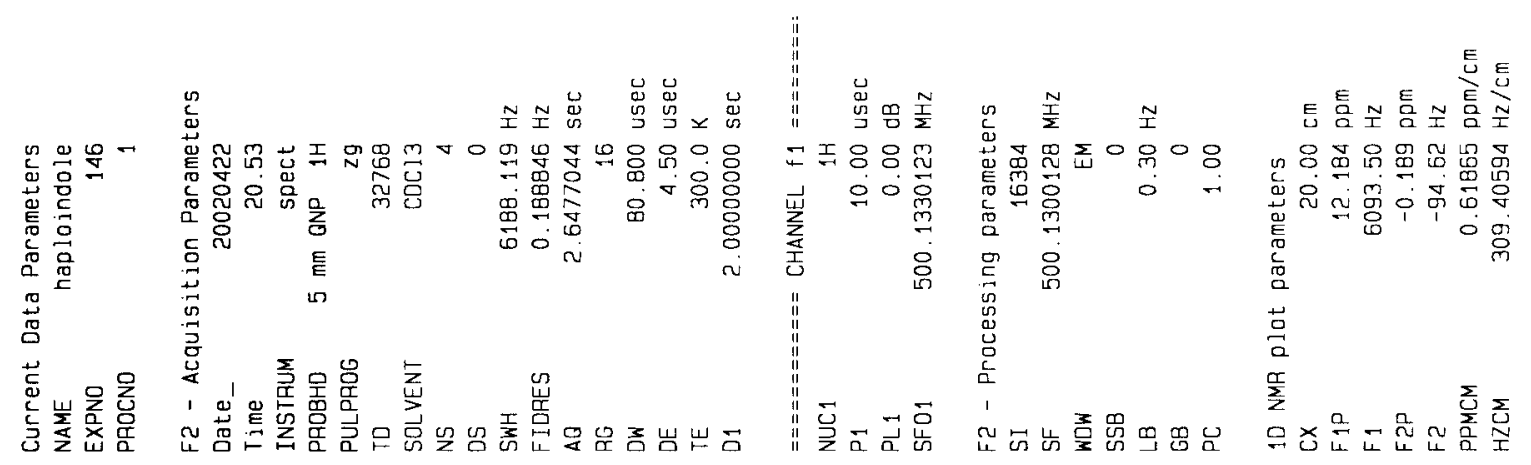

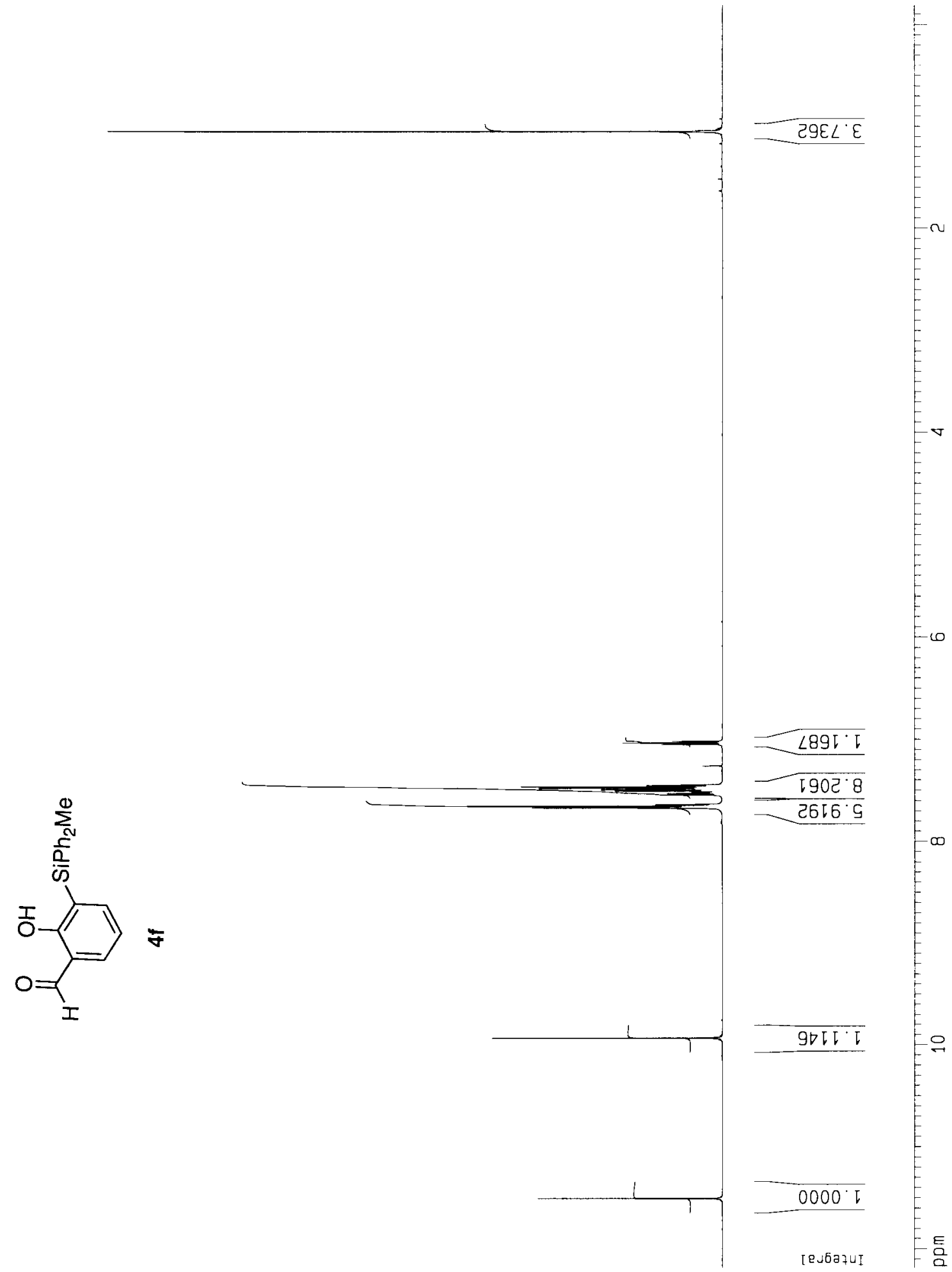



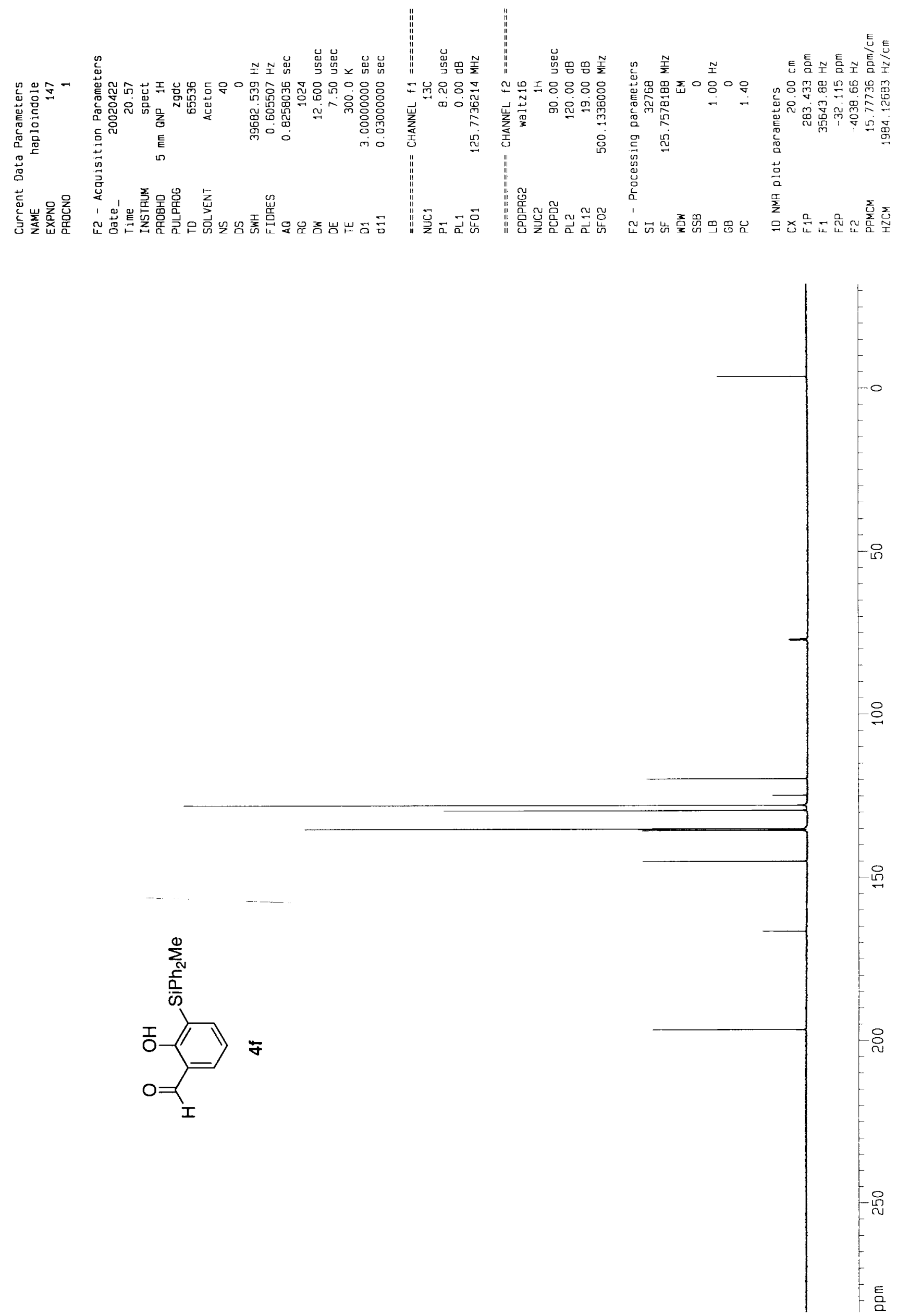

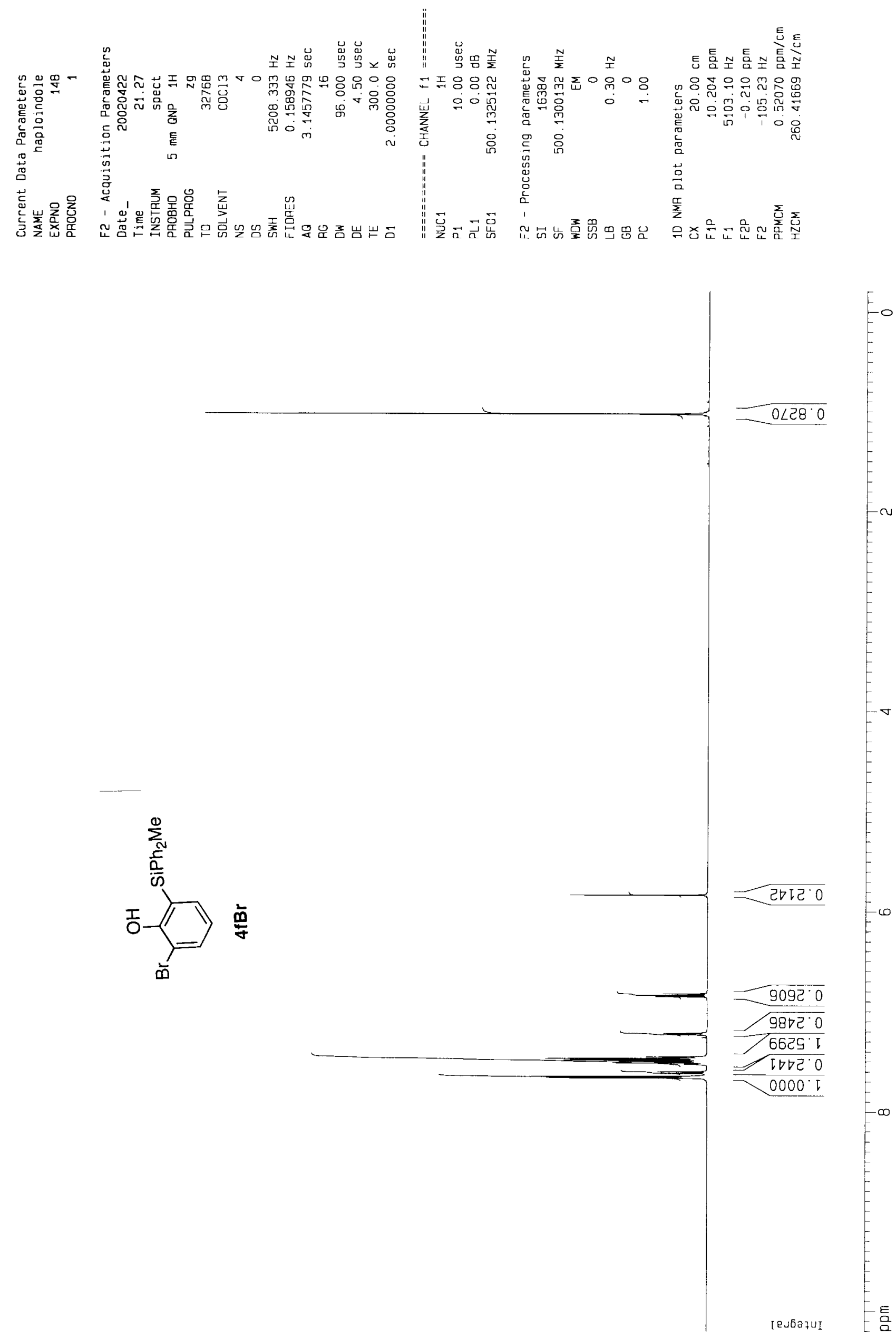

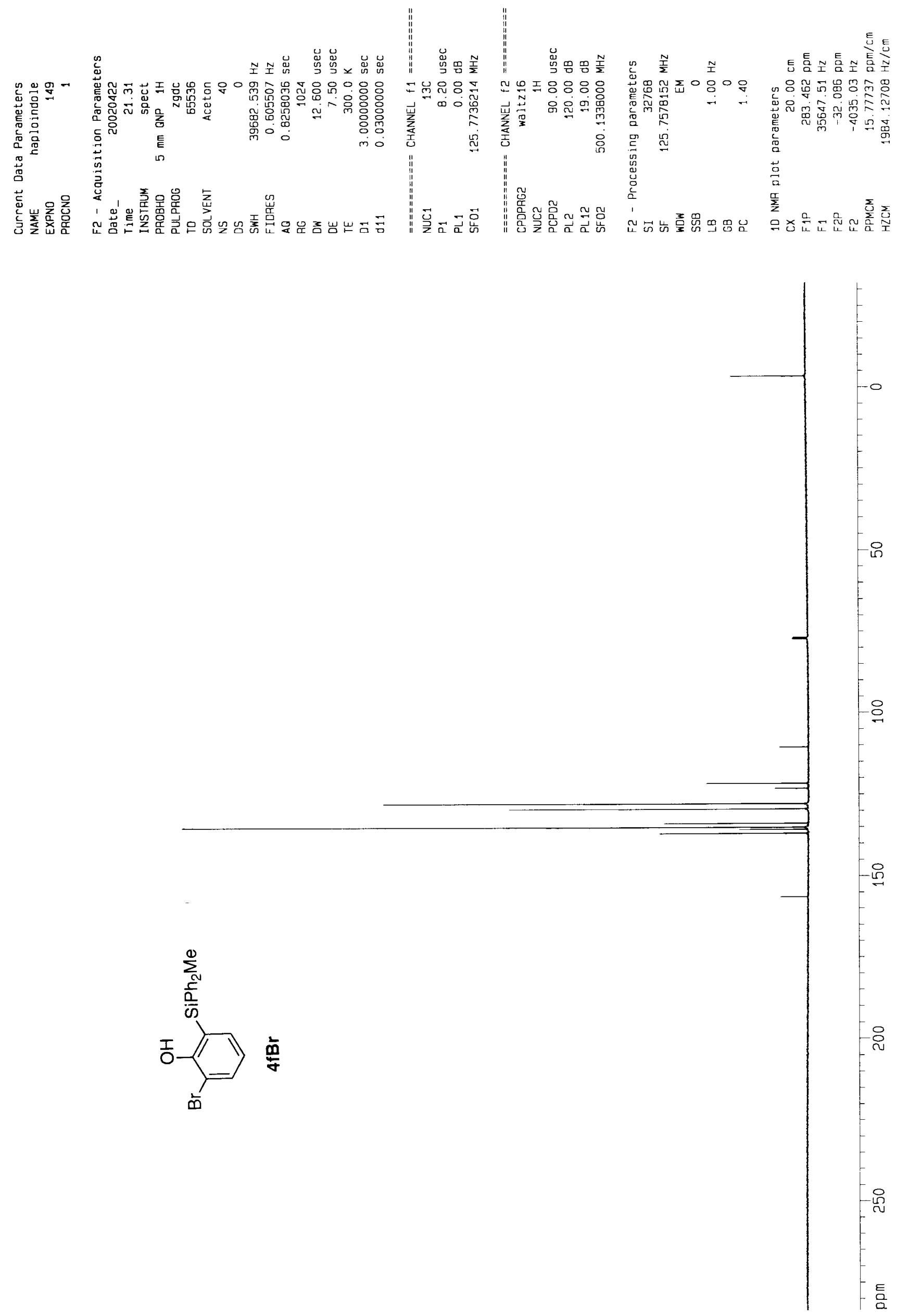


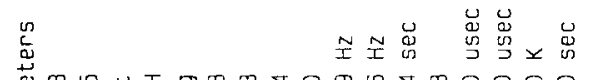

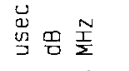

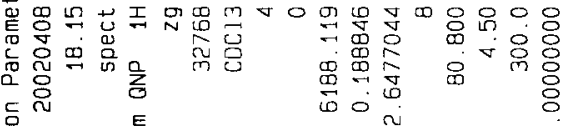

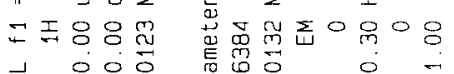

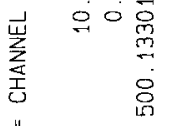

突

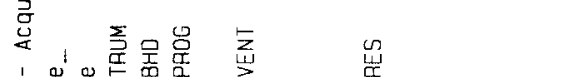

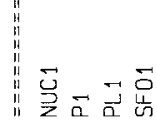

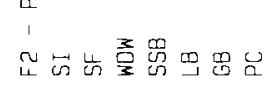

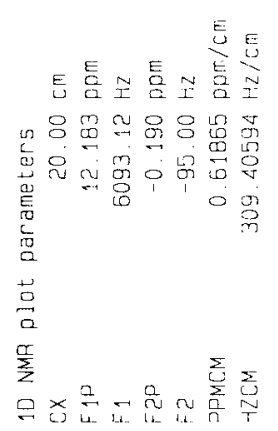

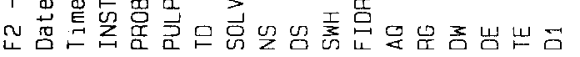
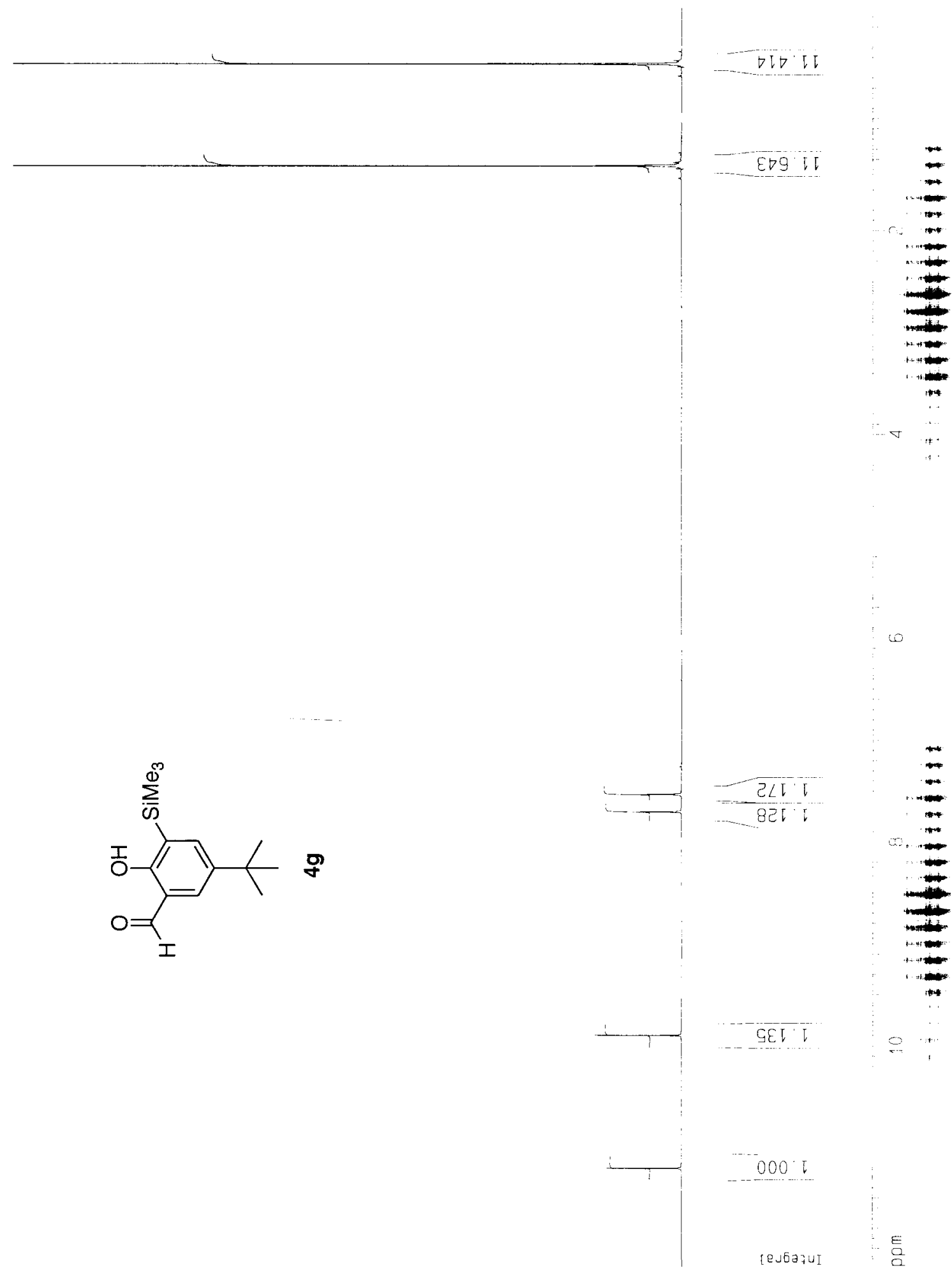


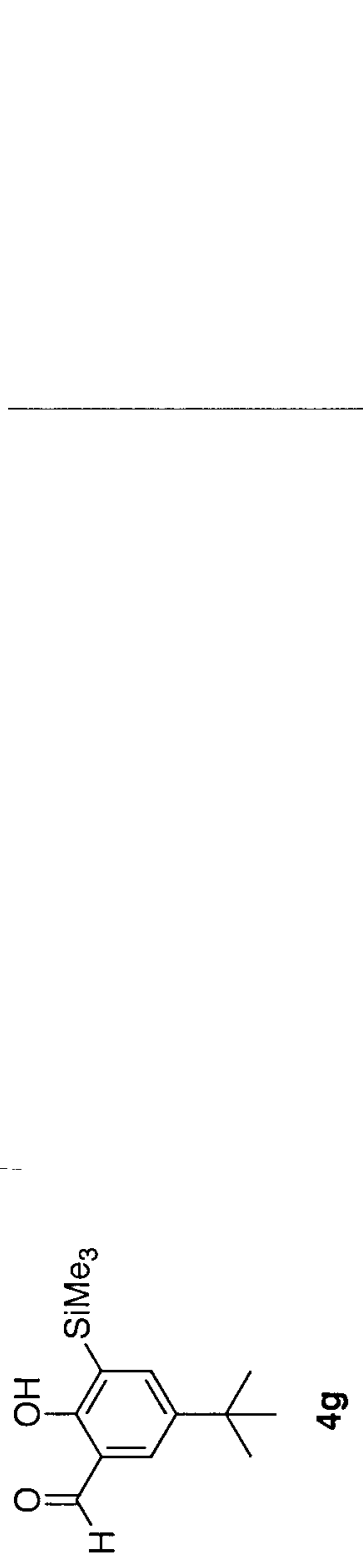




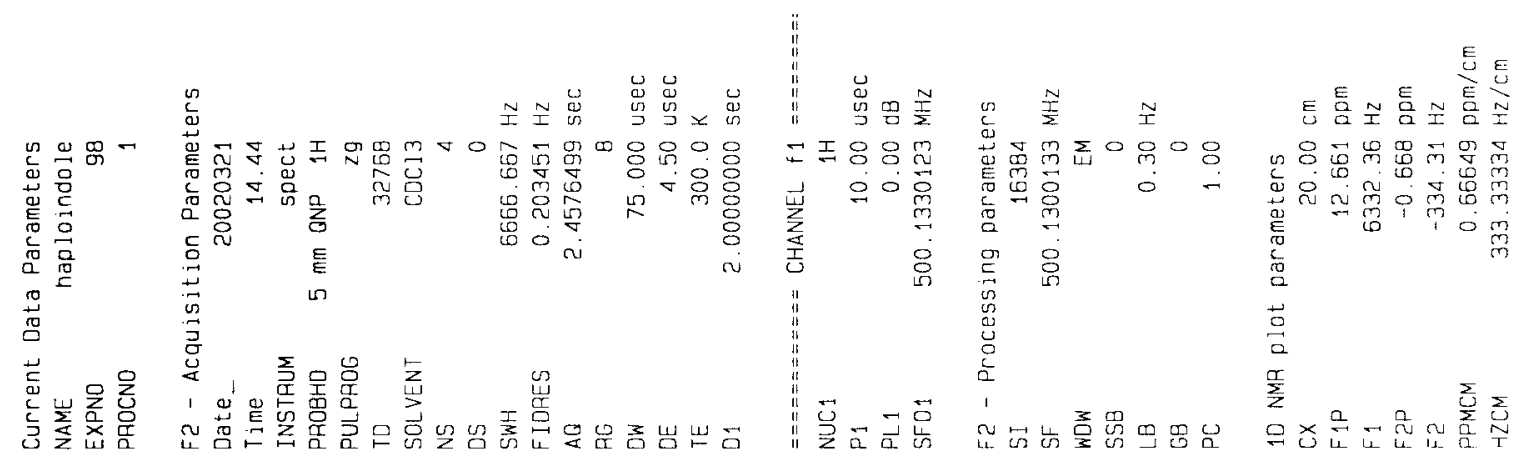
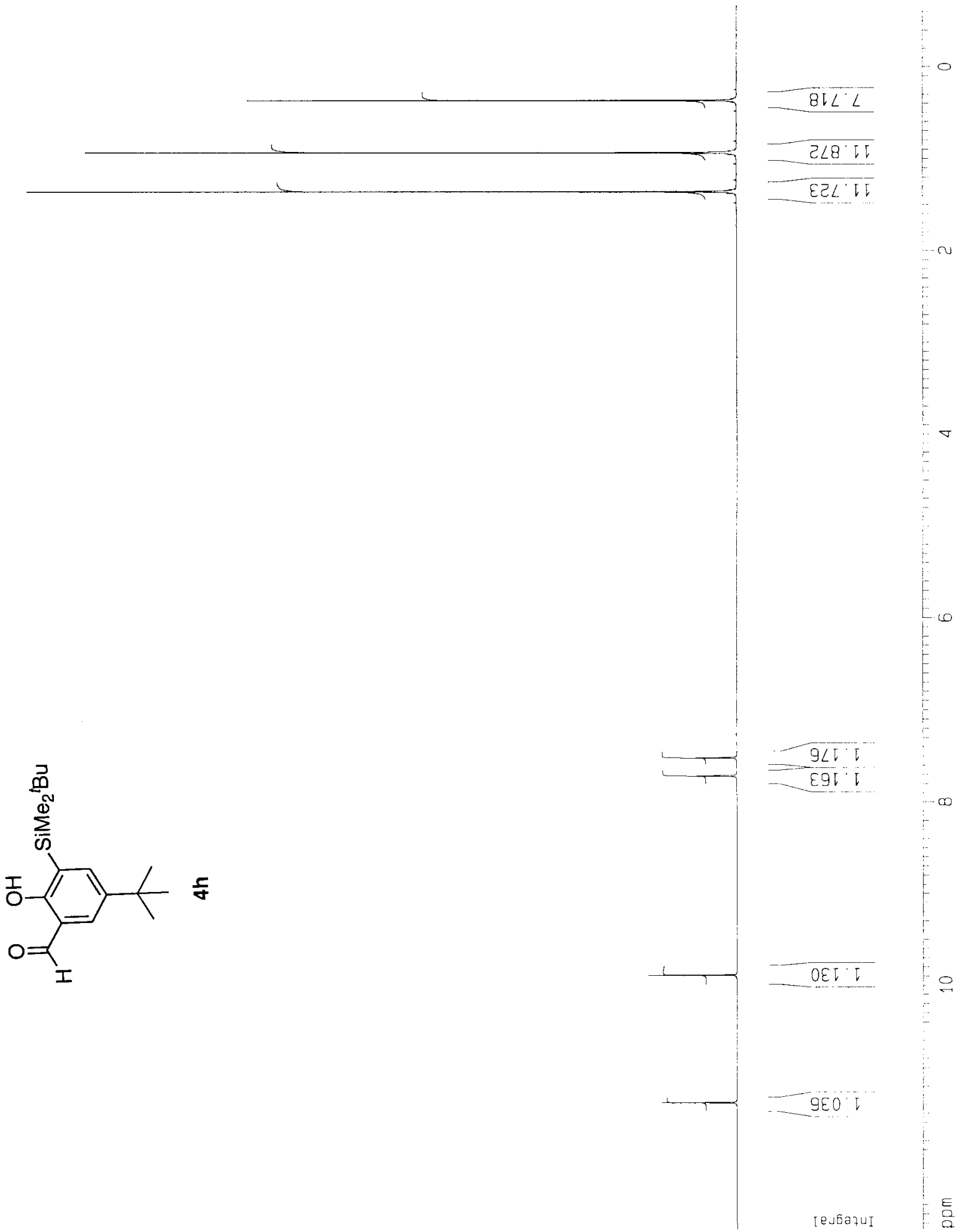


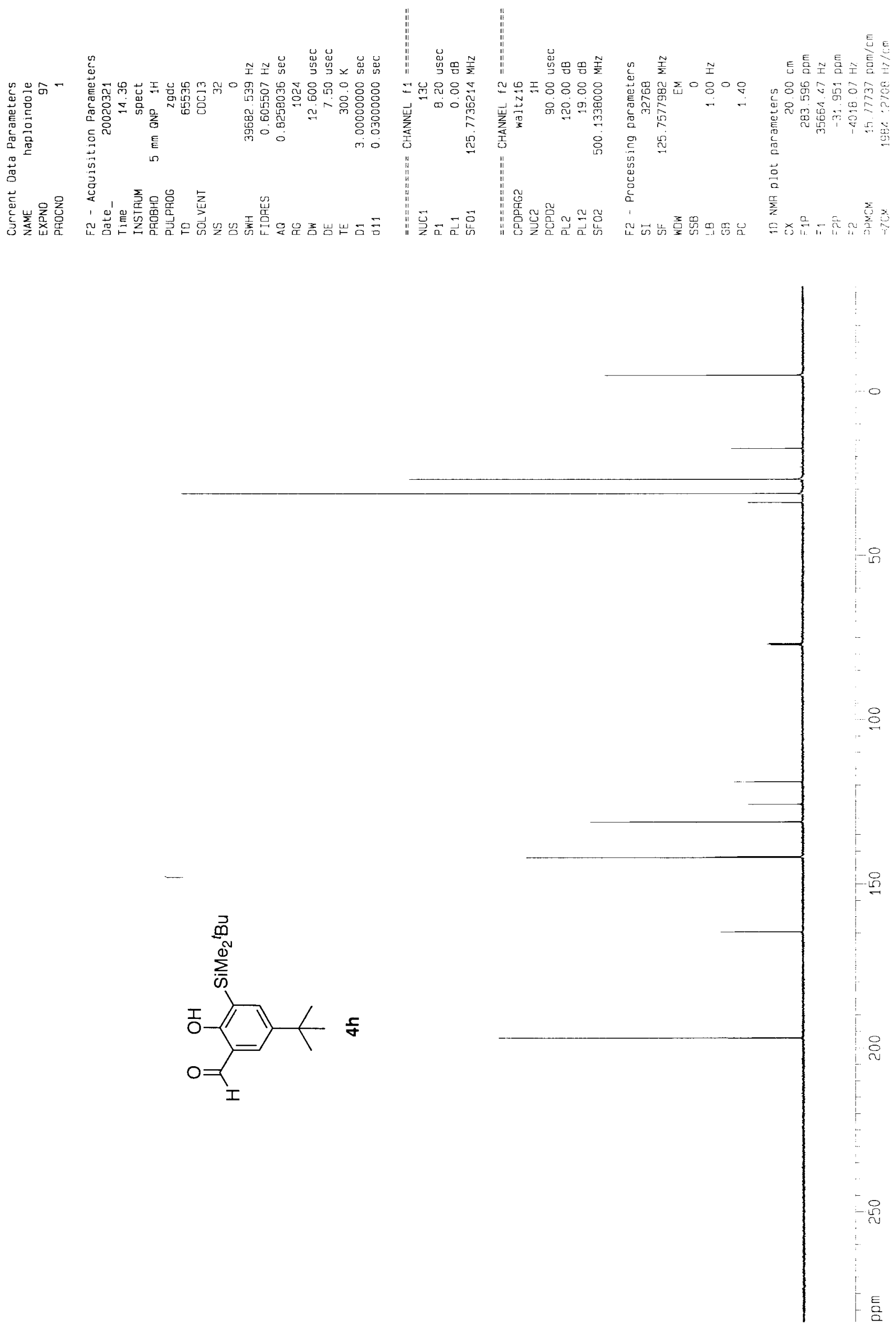



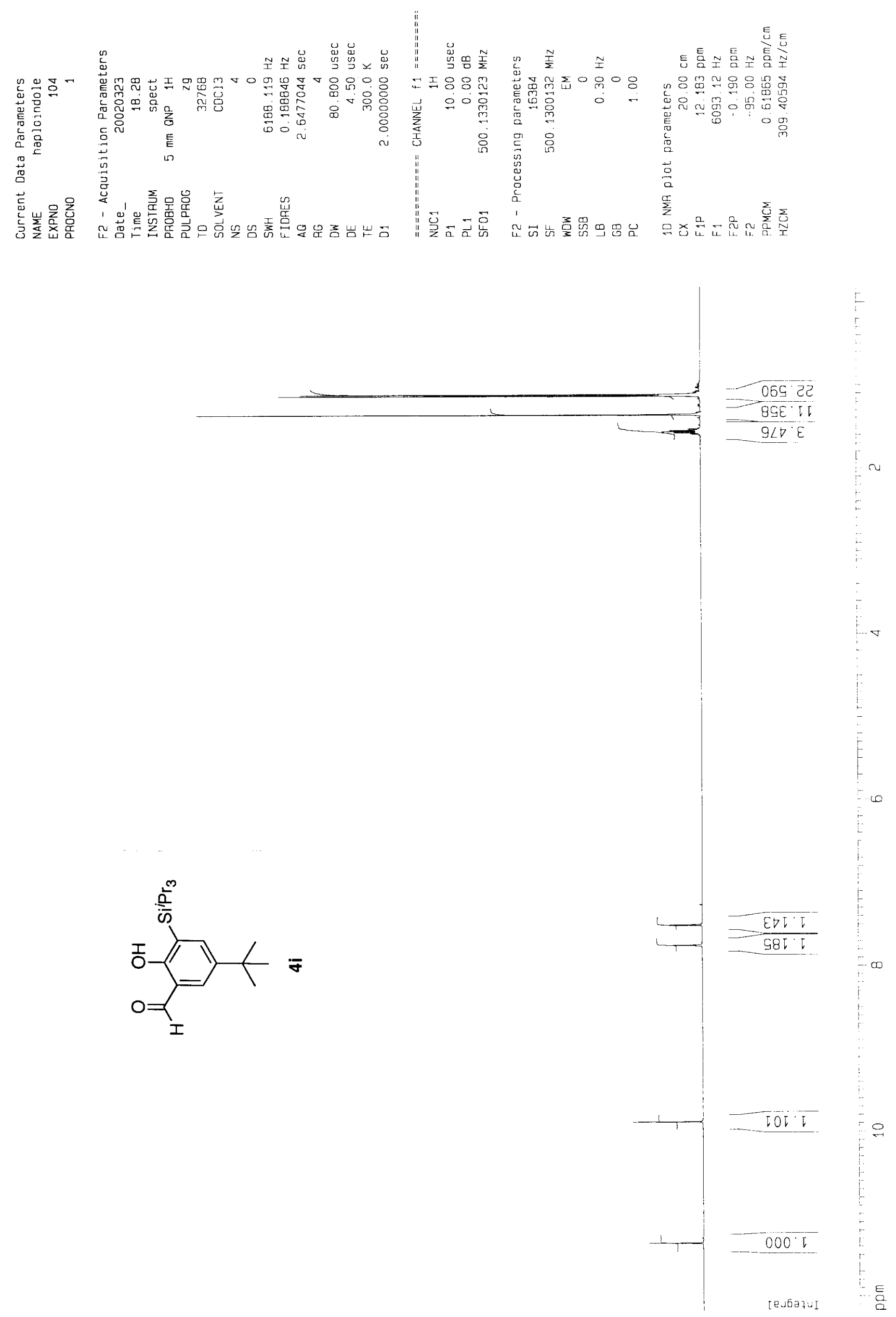


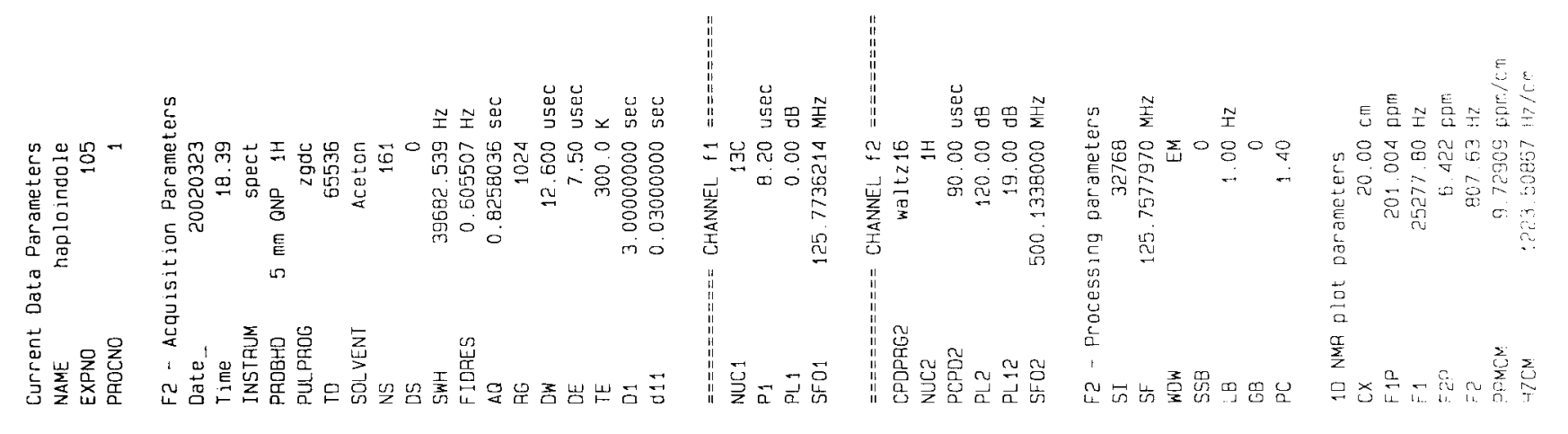

$809^{\prime}$ โ

$09 \angle 8$

LOC IE

$\triangle \angle 6^{\circ} E E$

600.61

$6 \angle \nabla^{\circ} \varepsilon 己 \downarrow$

$\nabla \nabla L O E L$

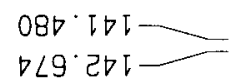

$966^{\circ} \triangleright 9 l$

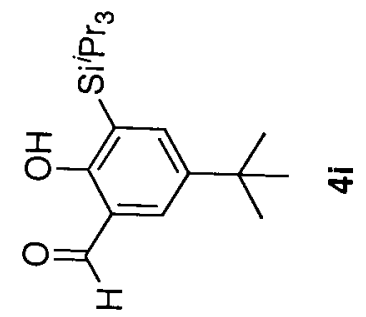

$886^{\circ} 961 \longrightarrow$

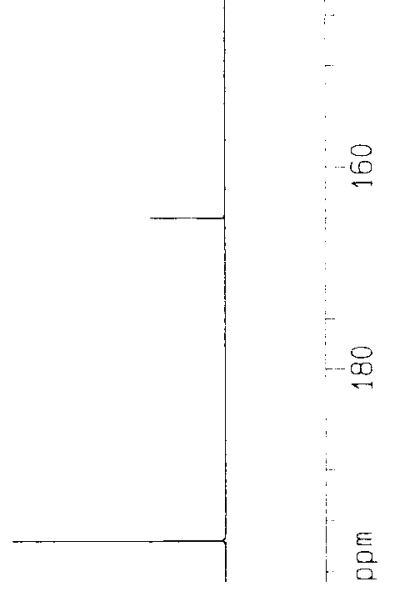




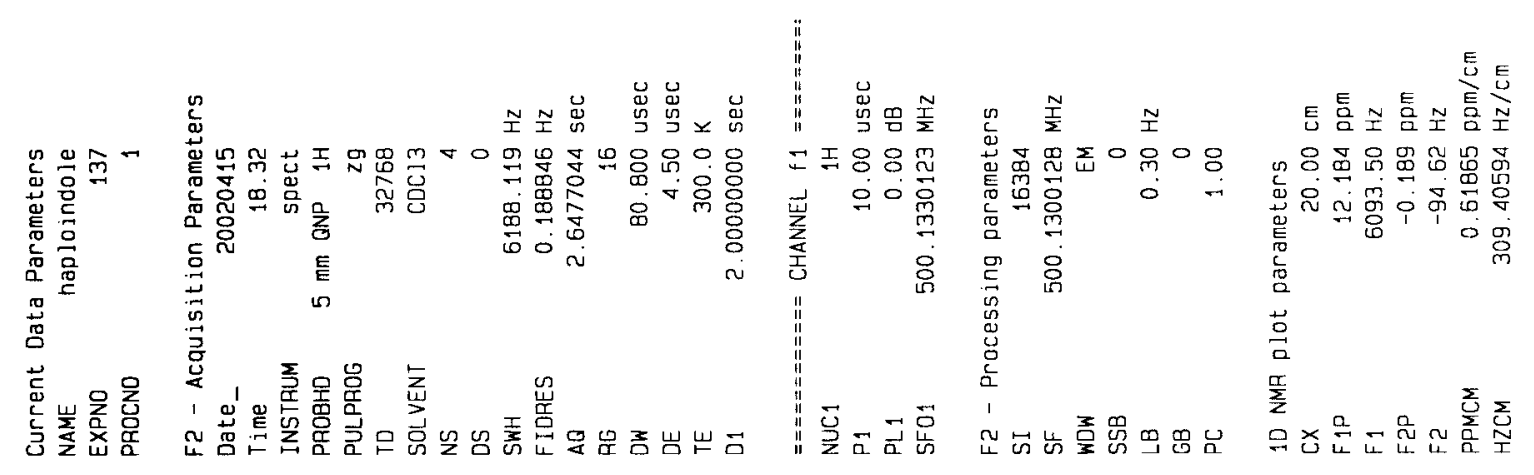

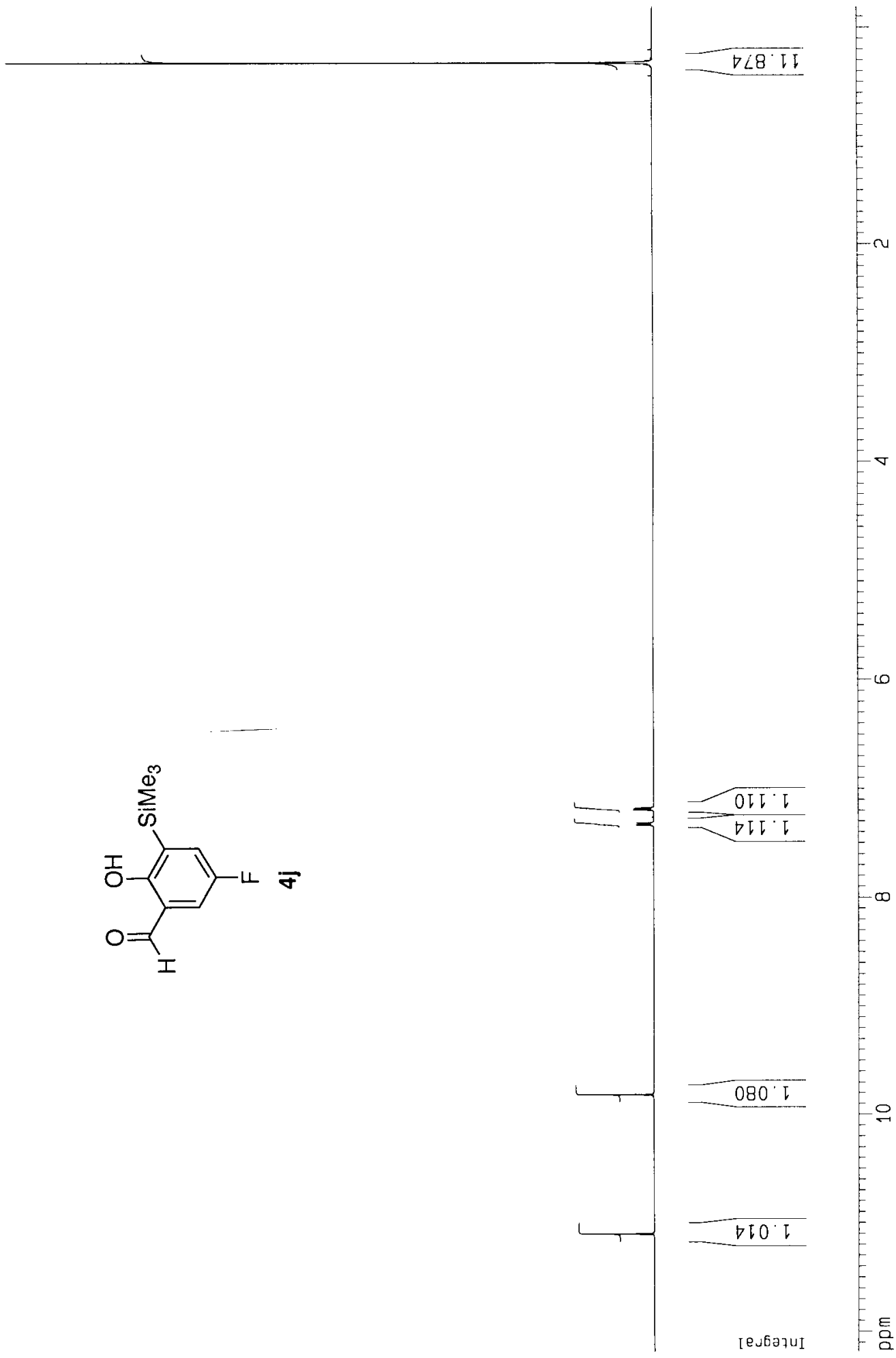




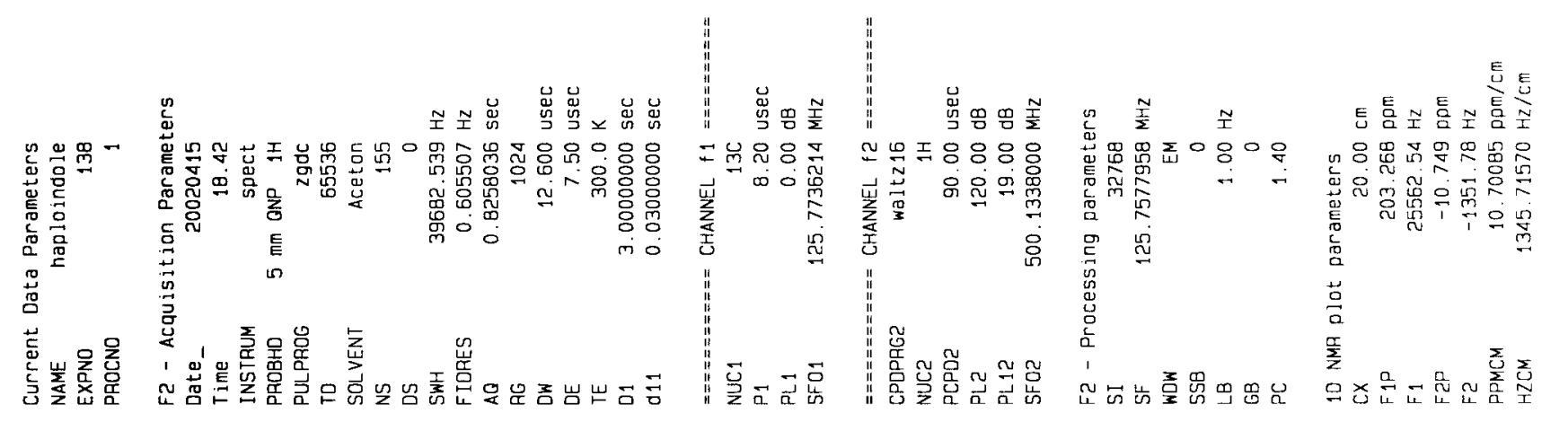

$\left\lceil\angle \mathcal{G}^{\prime} \downarrow-\right.$
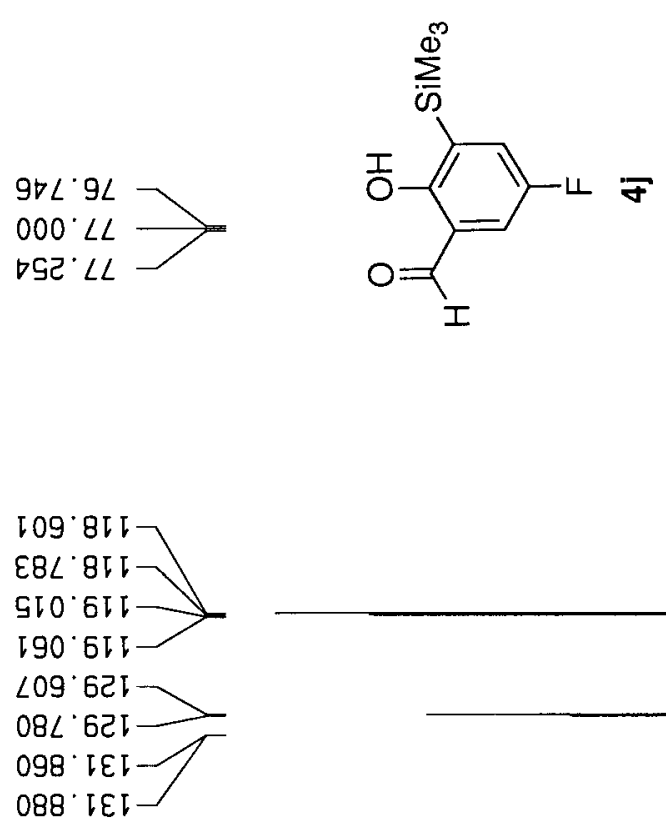

$\nabla 99^{\circ} \nabla G I-$
$8 \angle 9^{\circ}$ 9SI

ટЕ己· 291

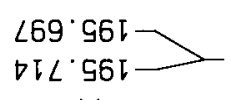



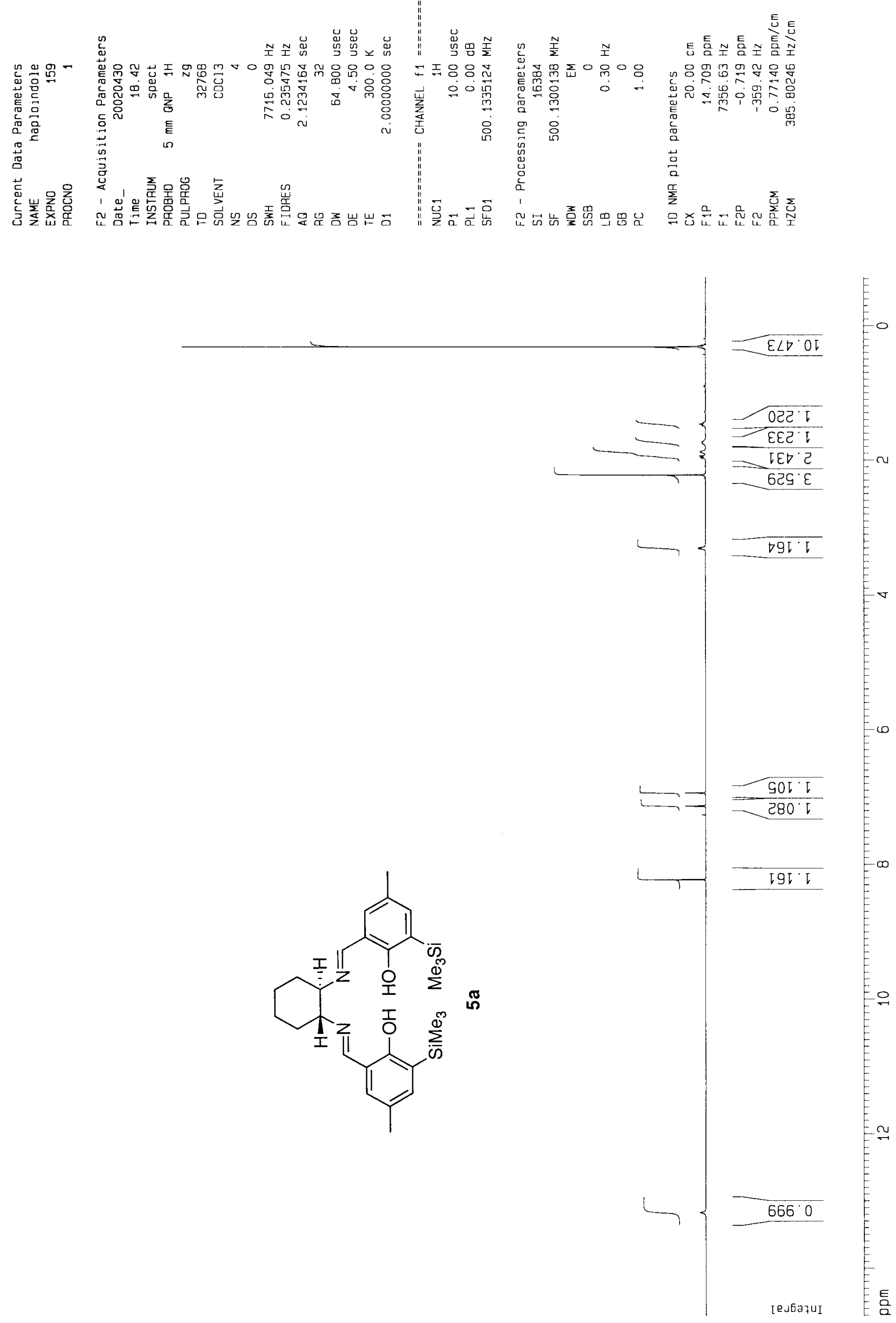


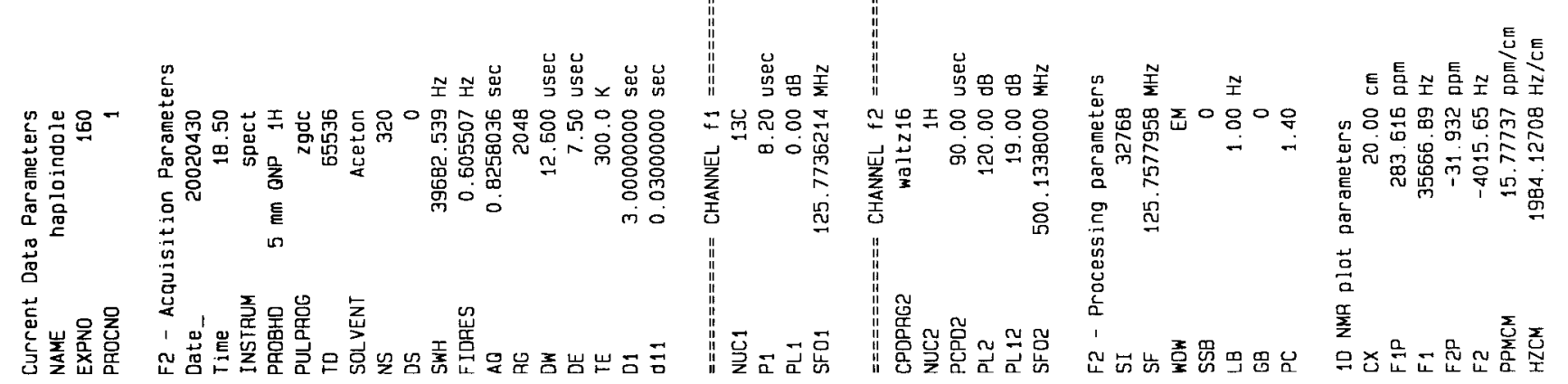

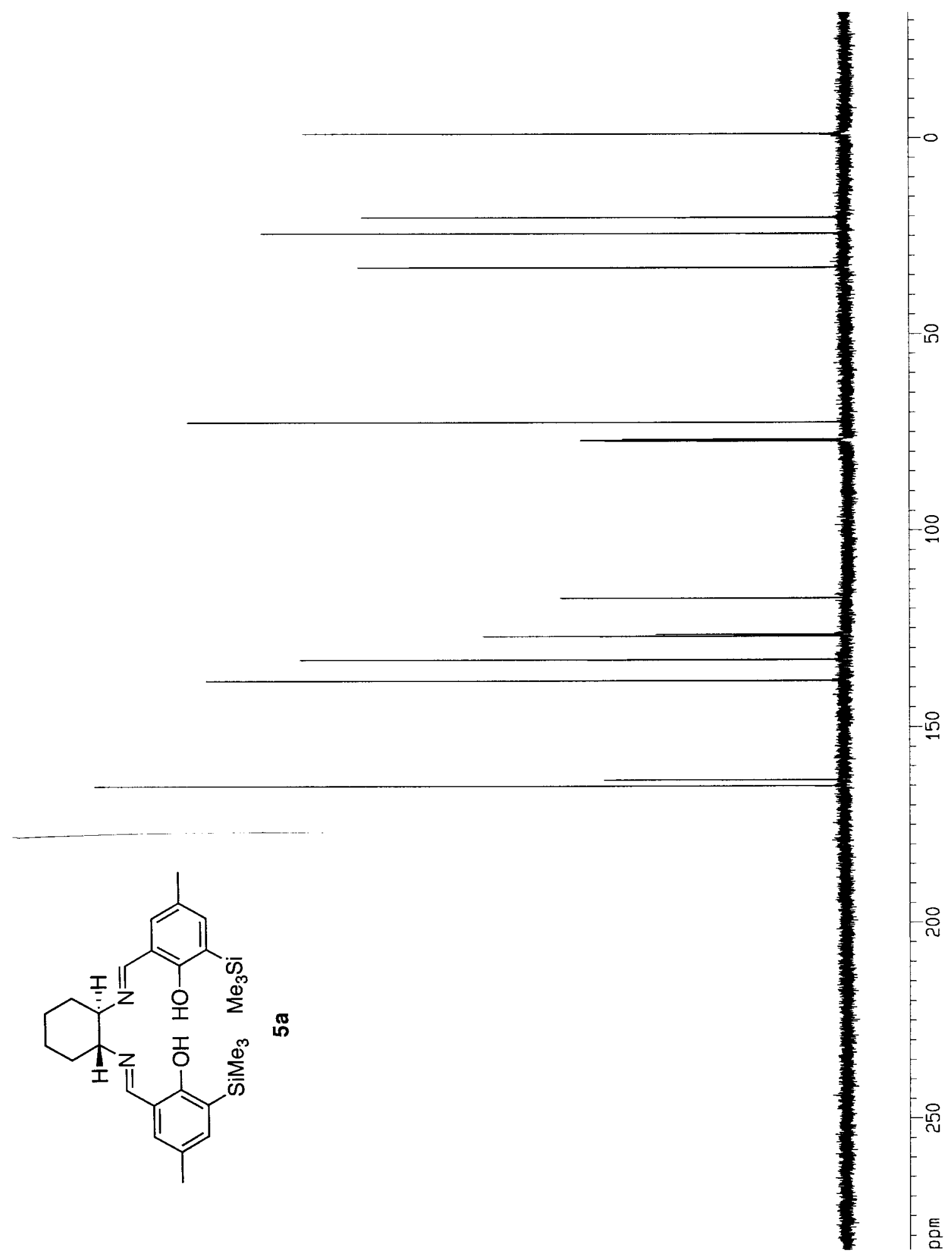




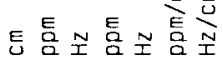

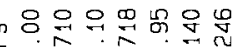

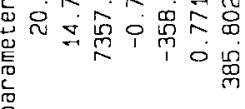
苔

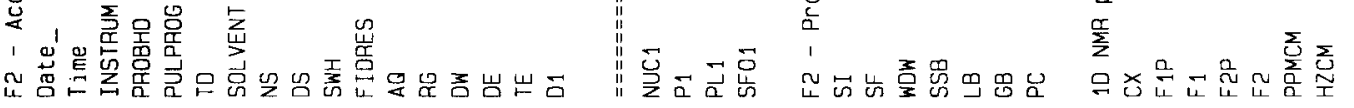

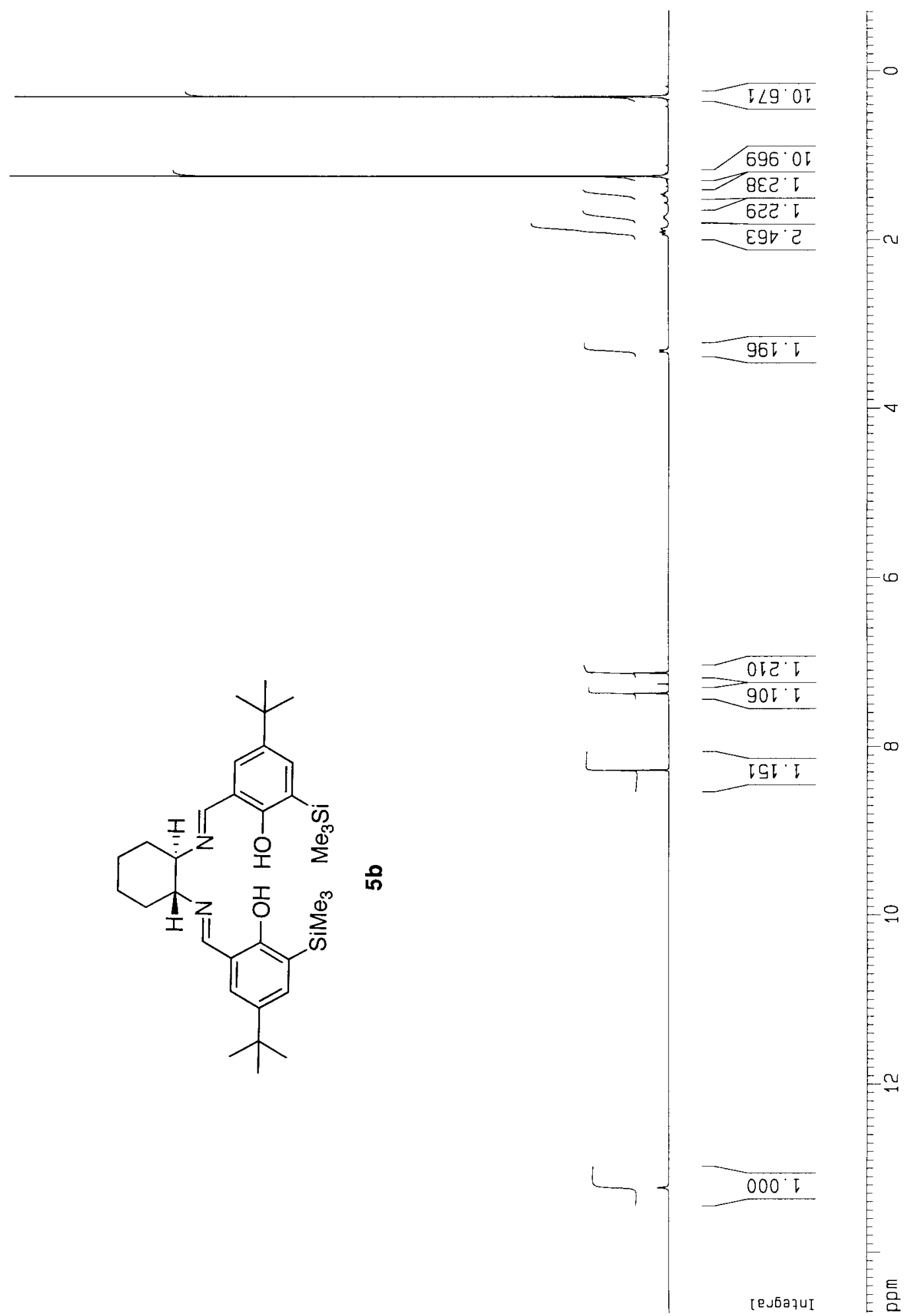




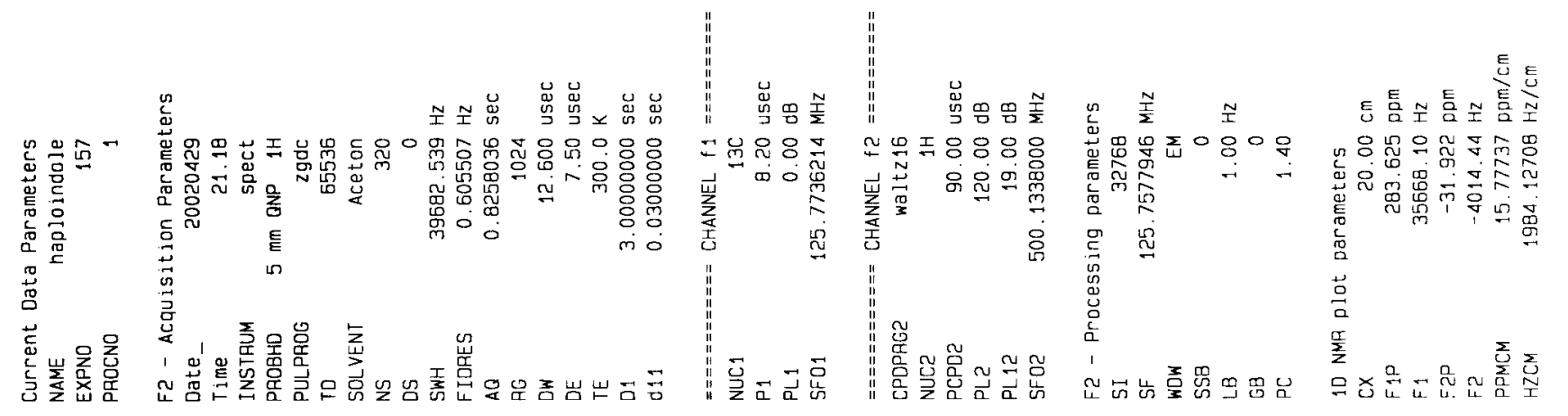
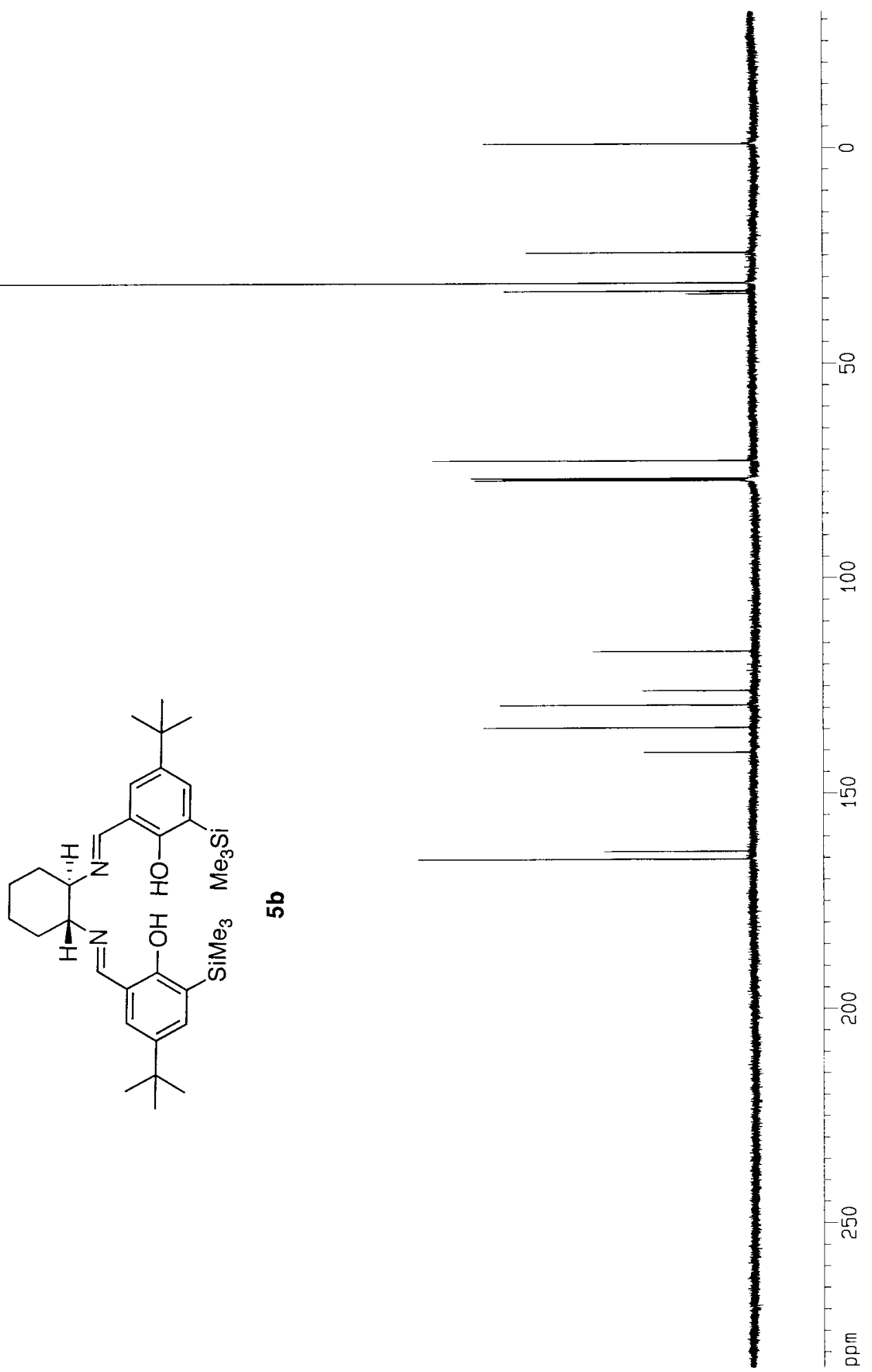

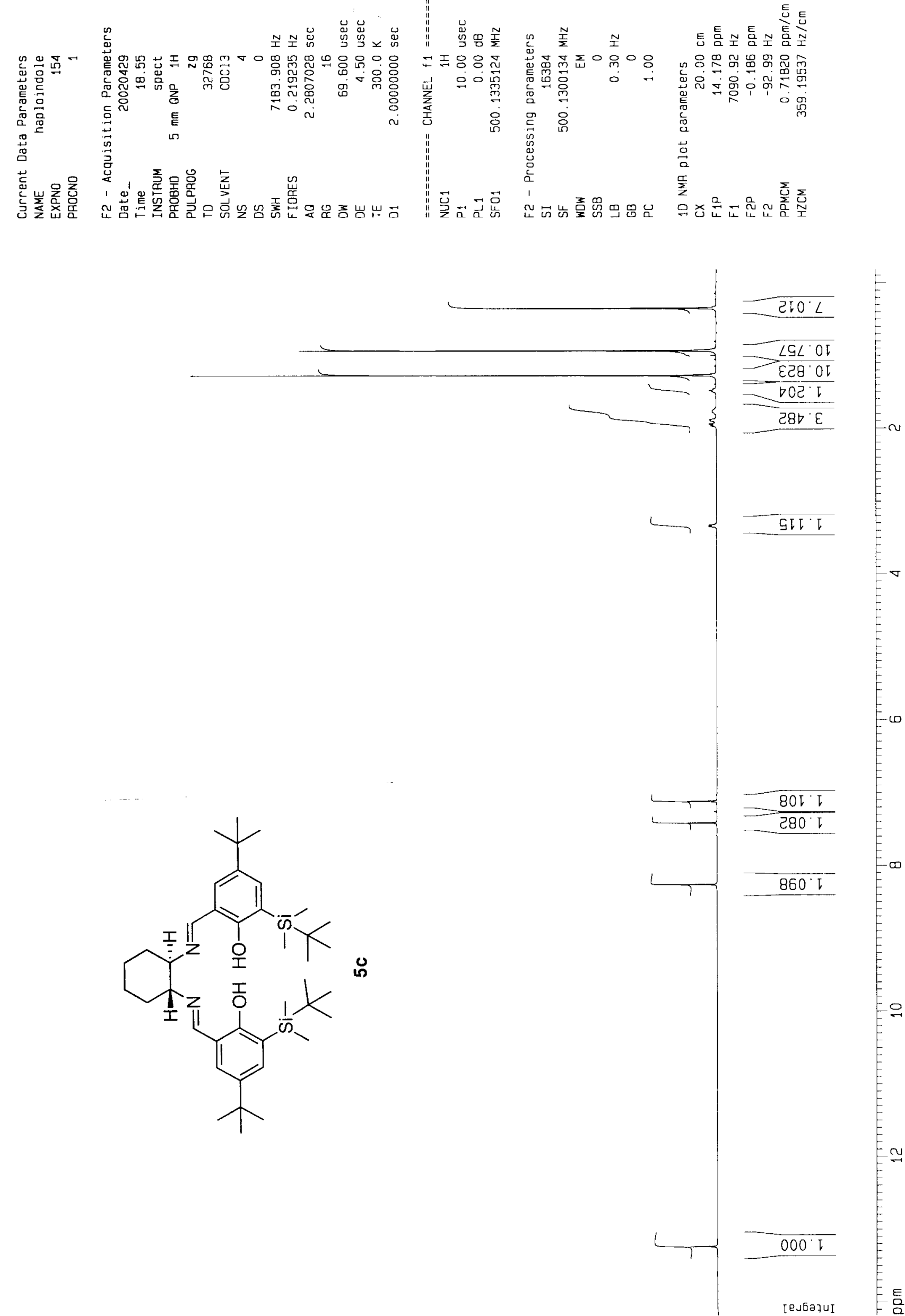

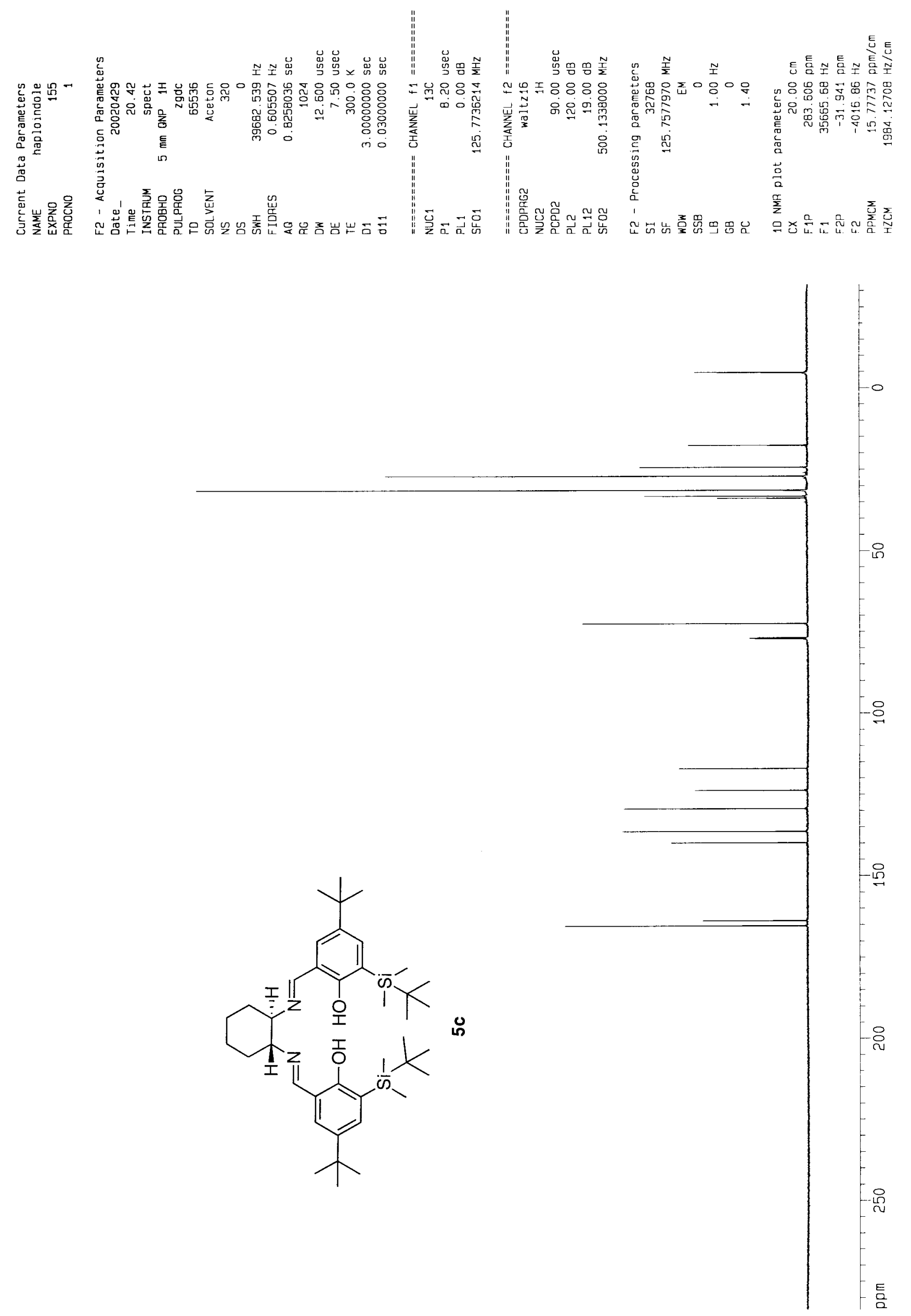

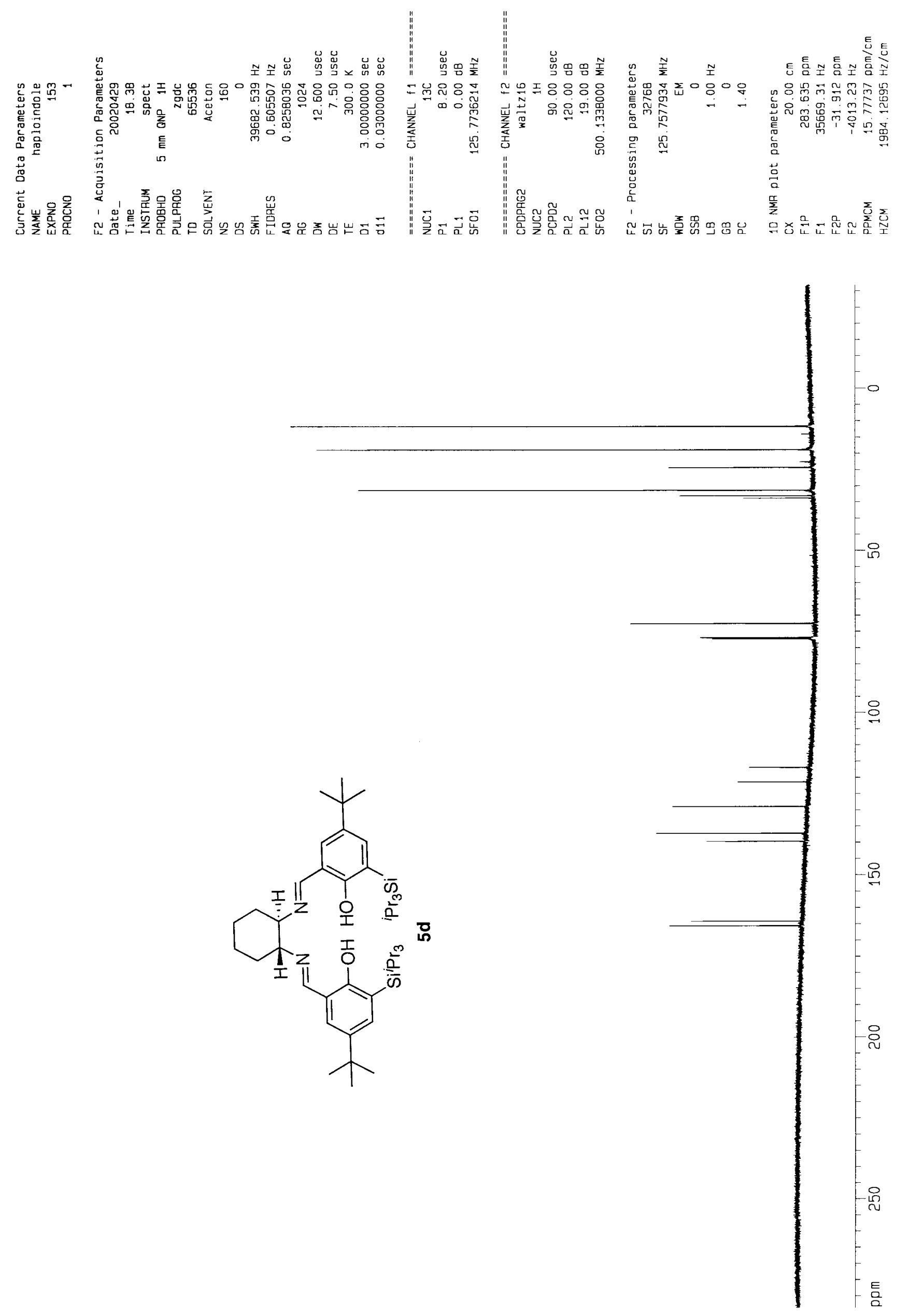


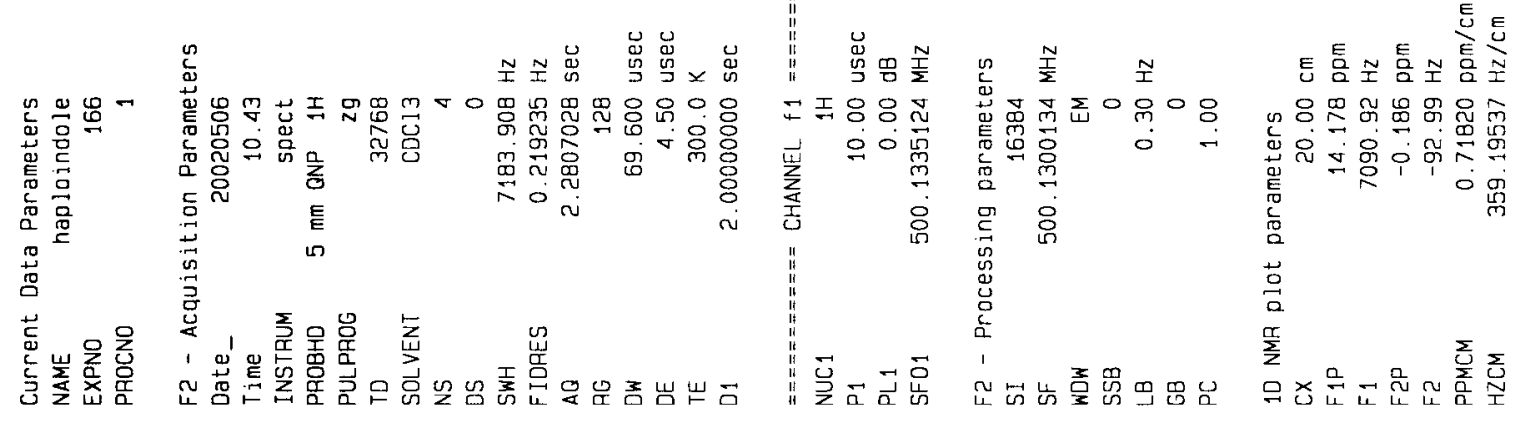

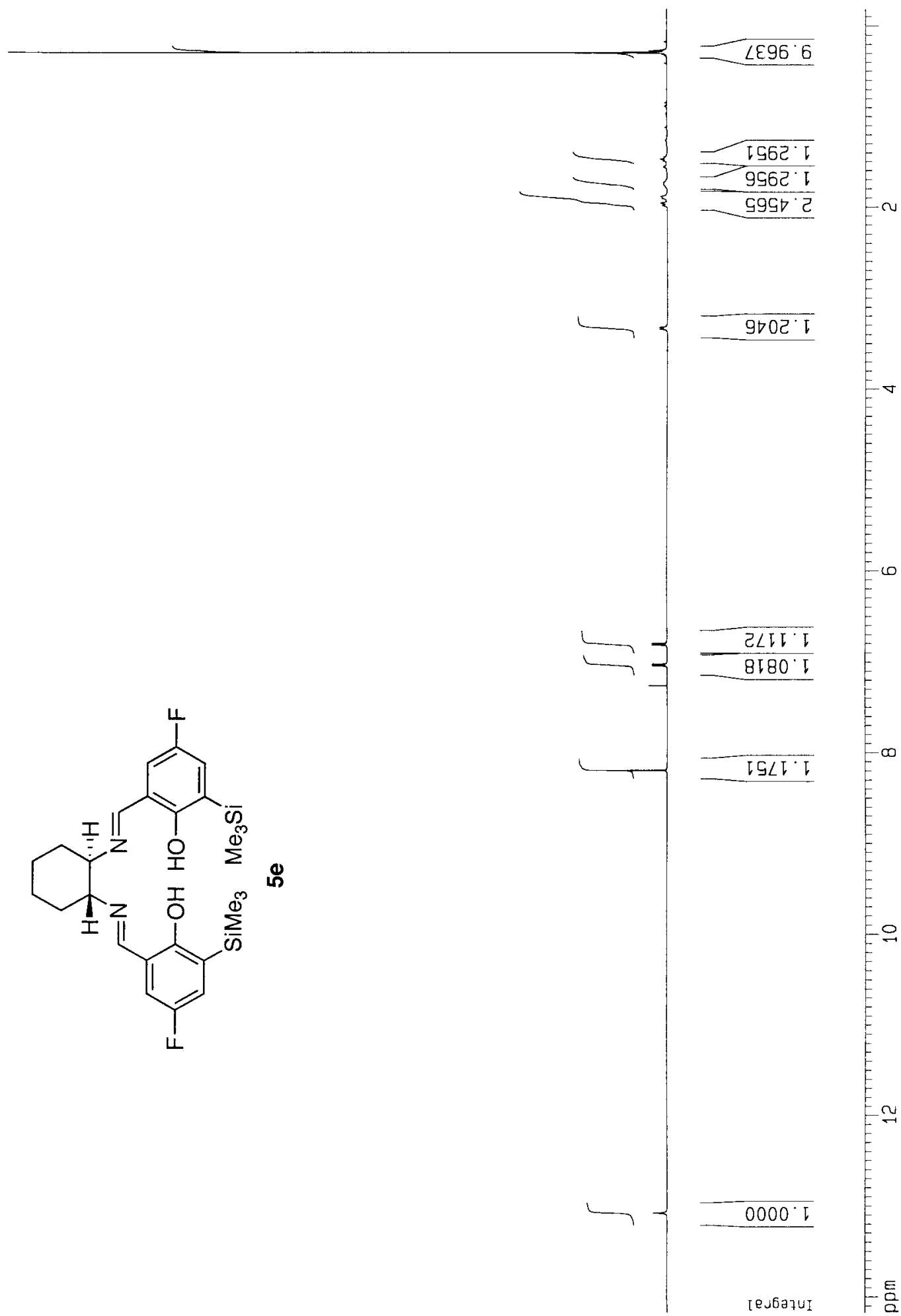




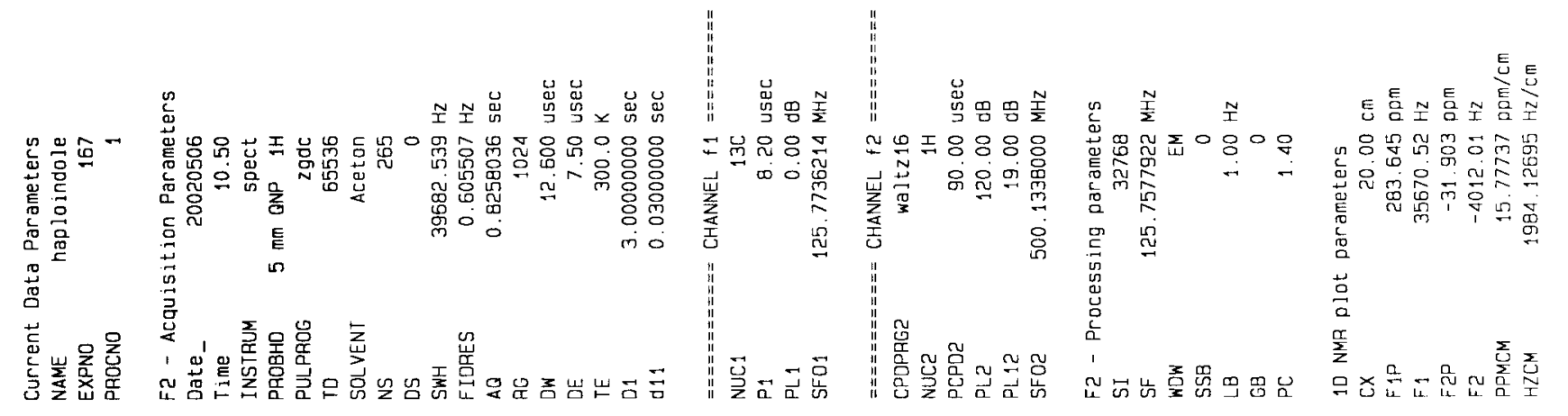

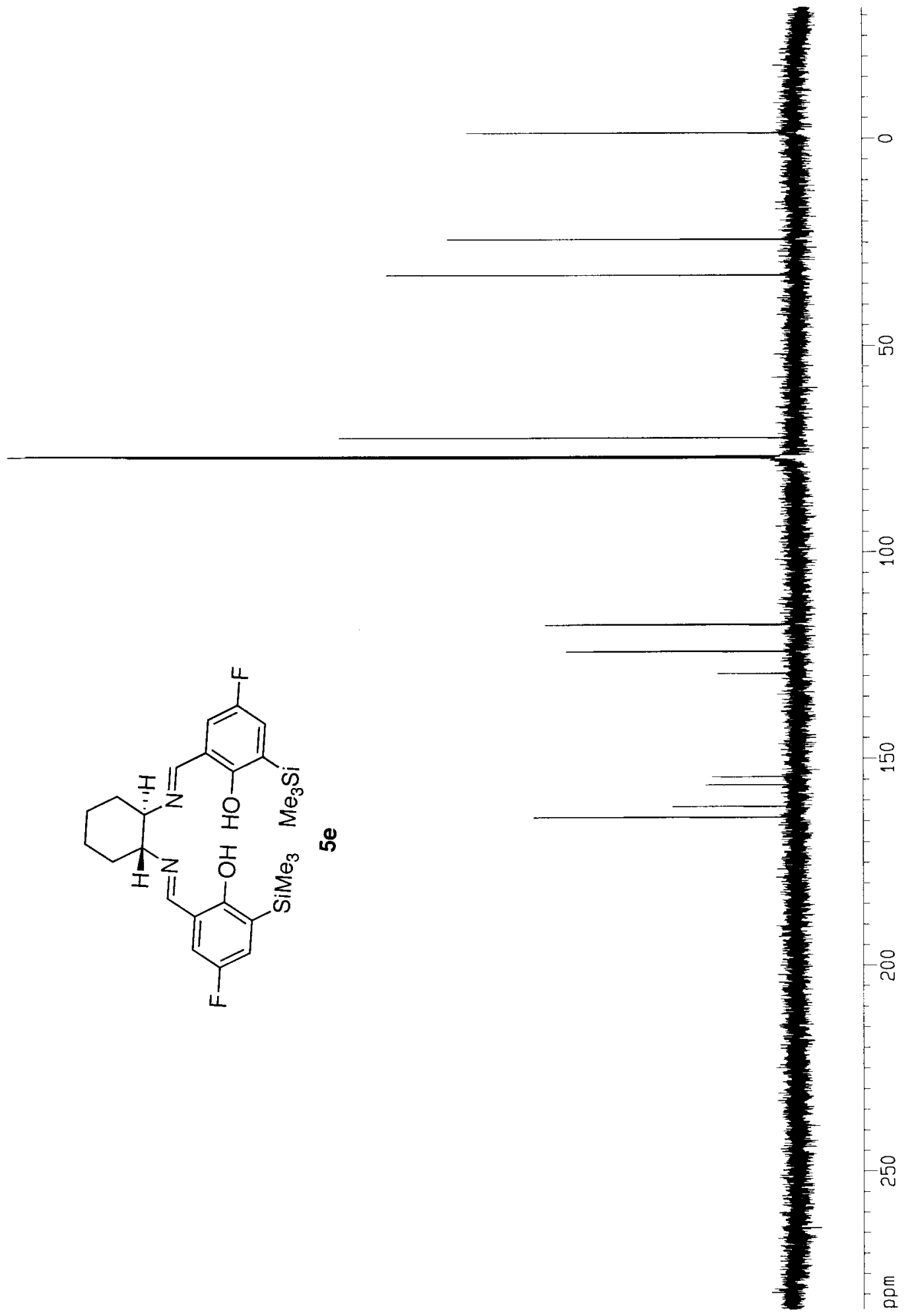




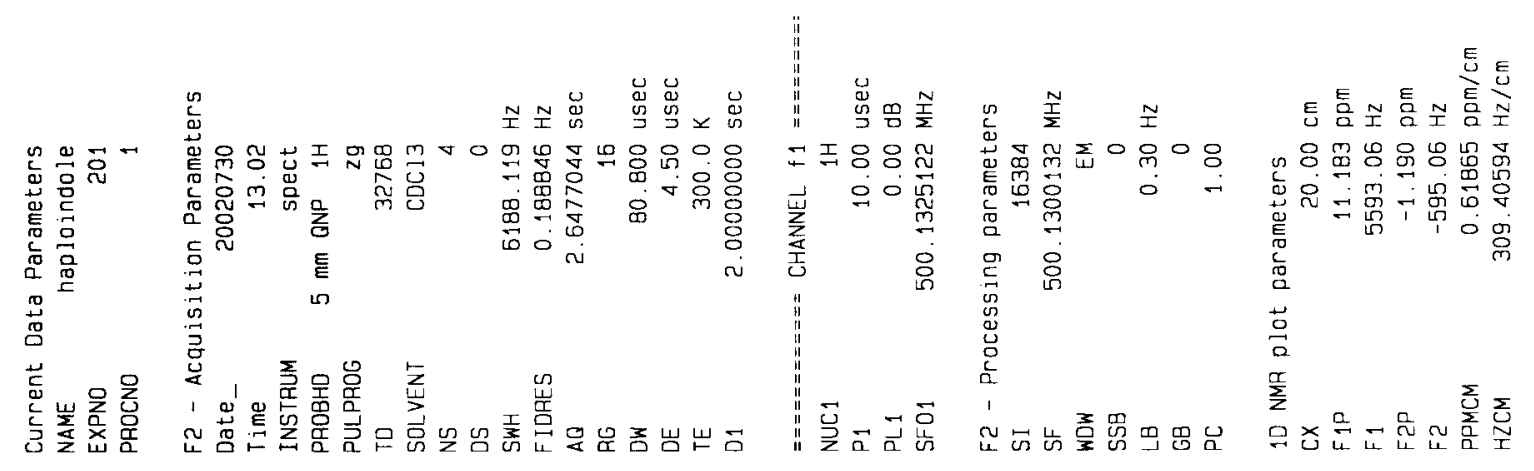

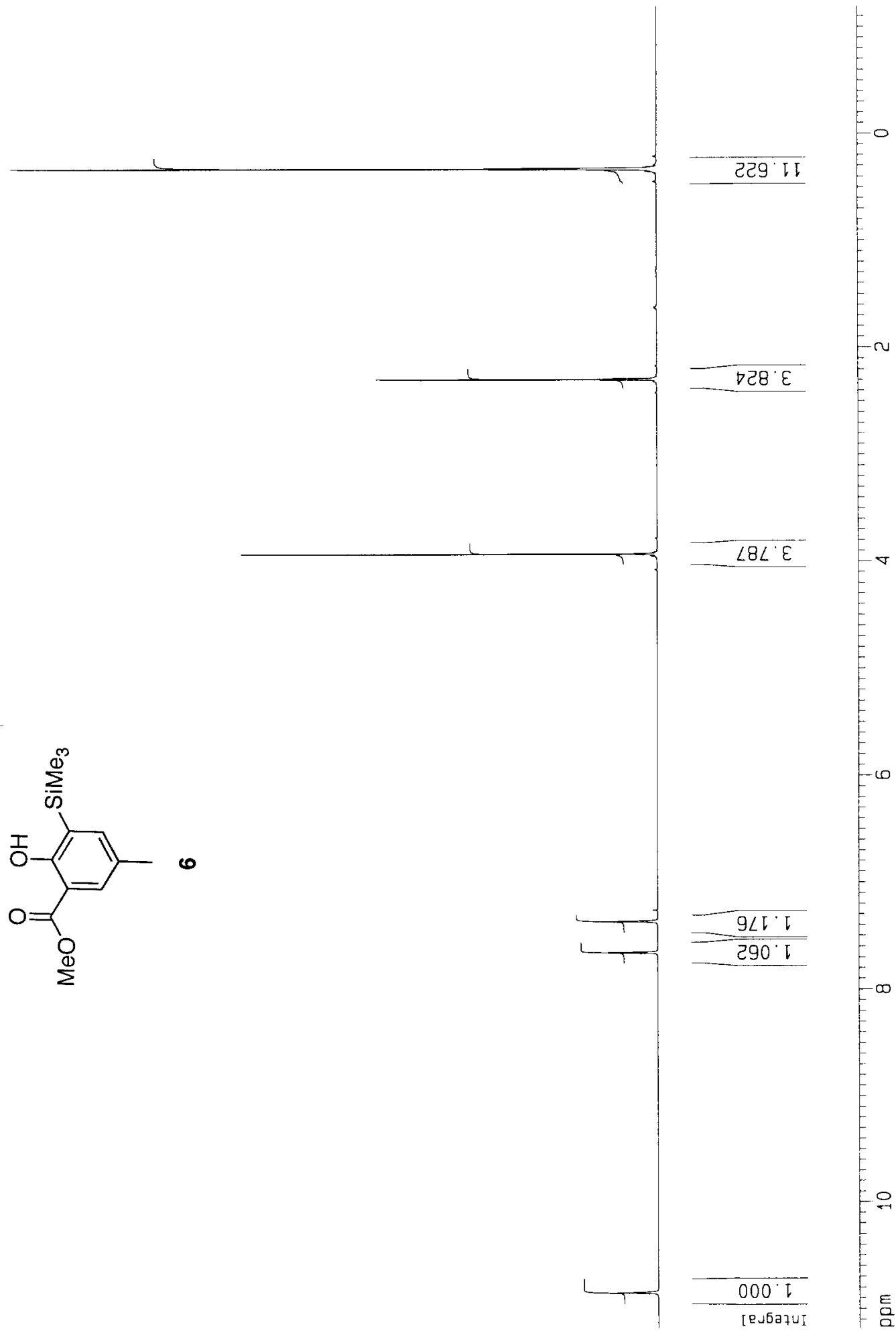




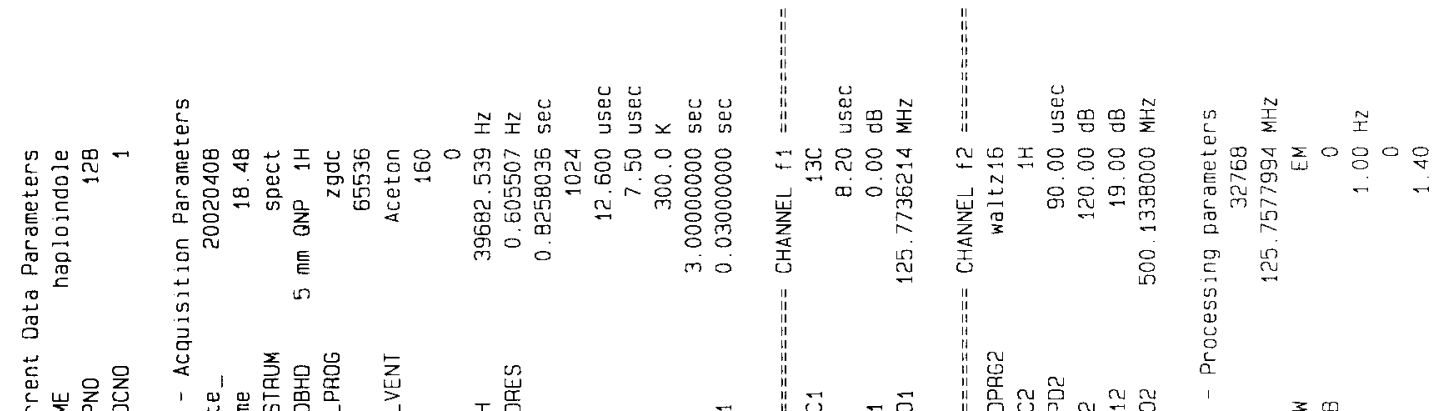

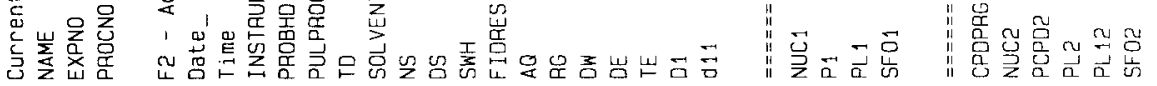

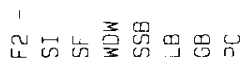

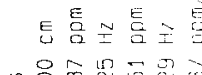

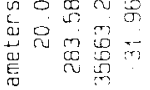

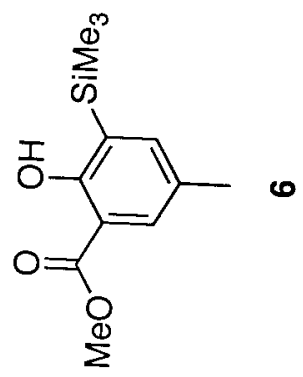




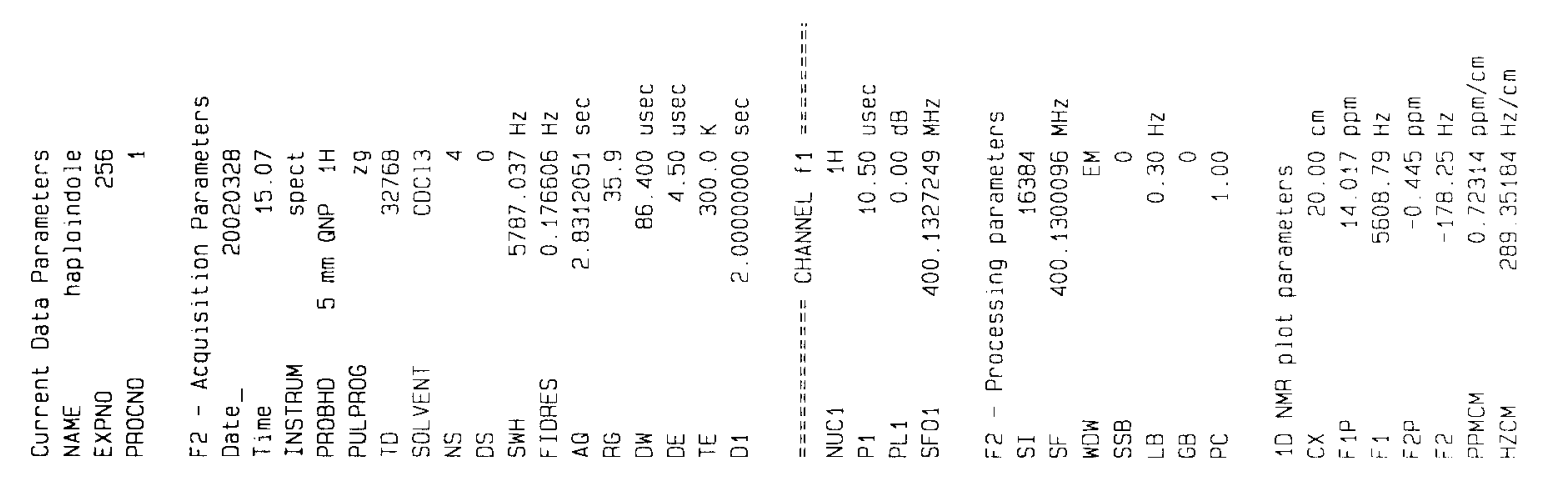

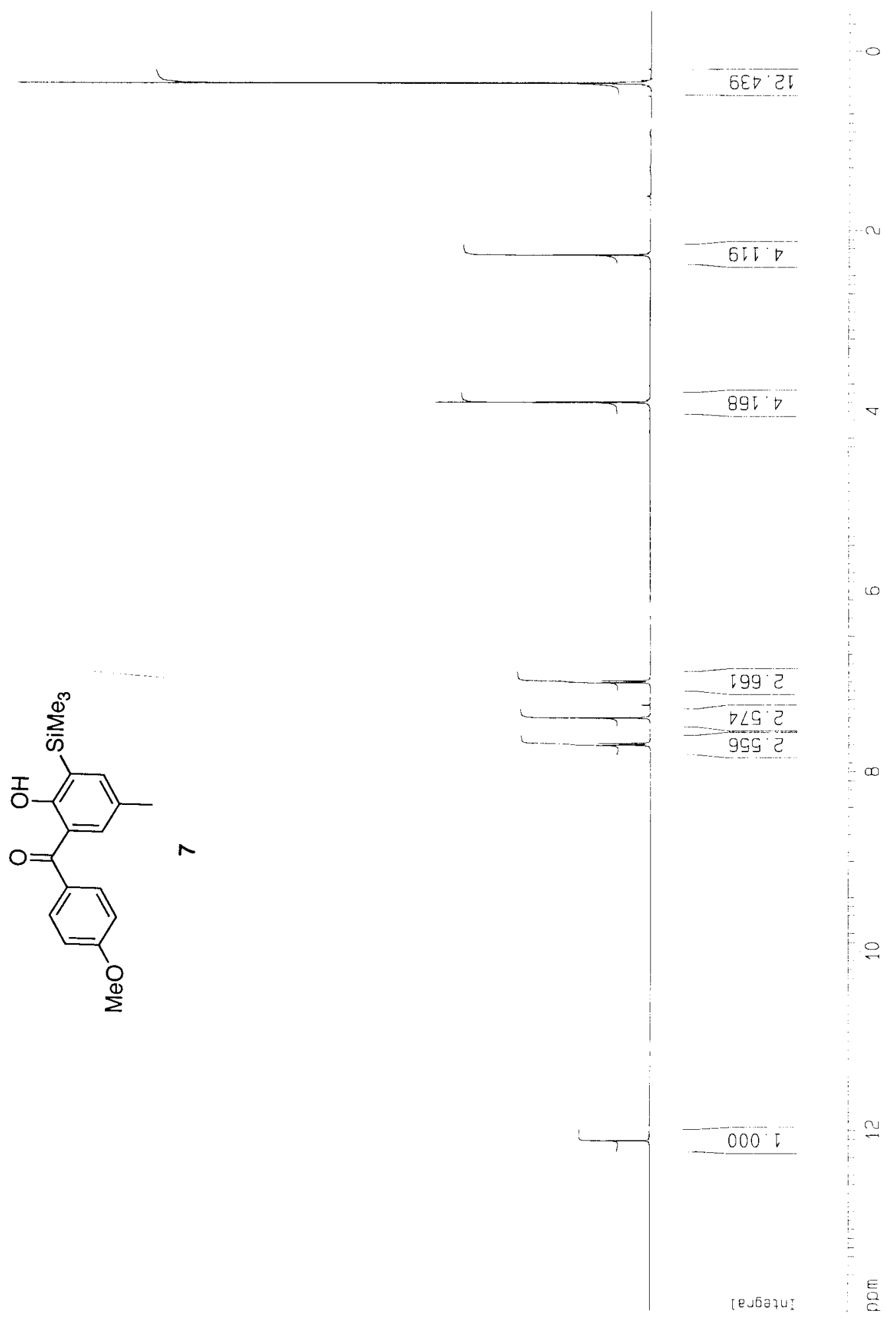




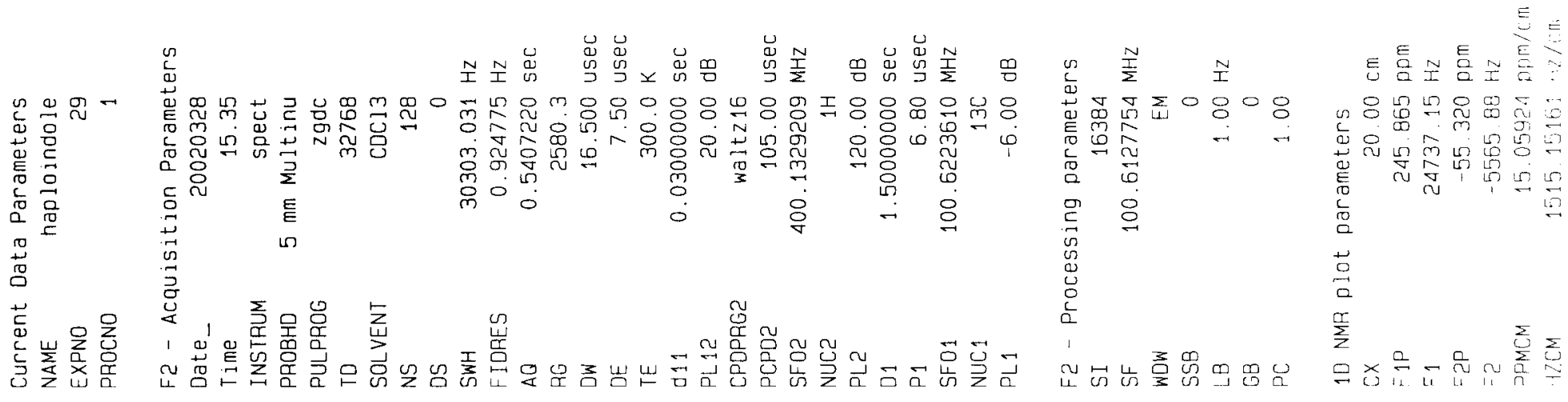

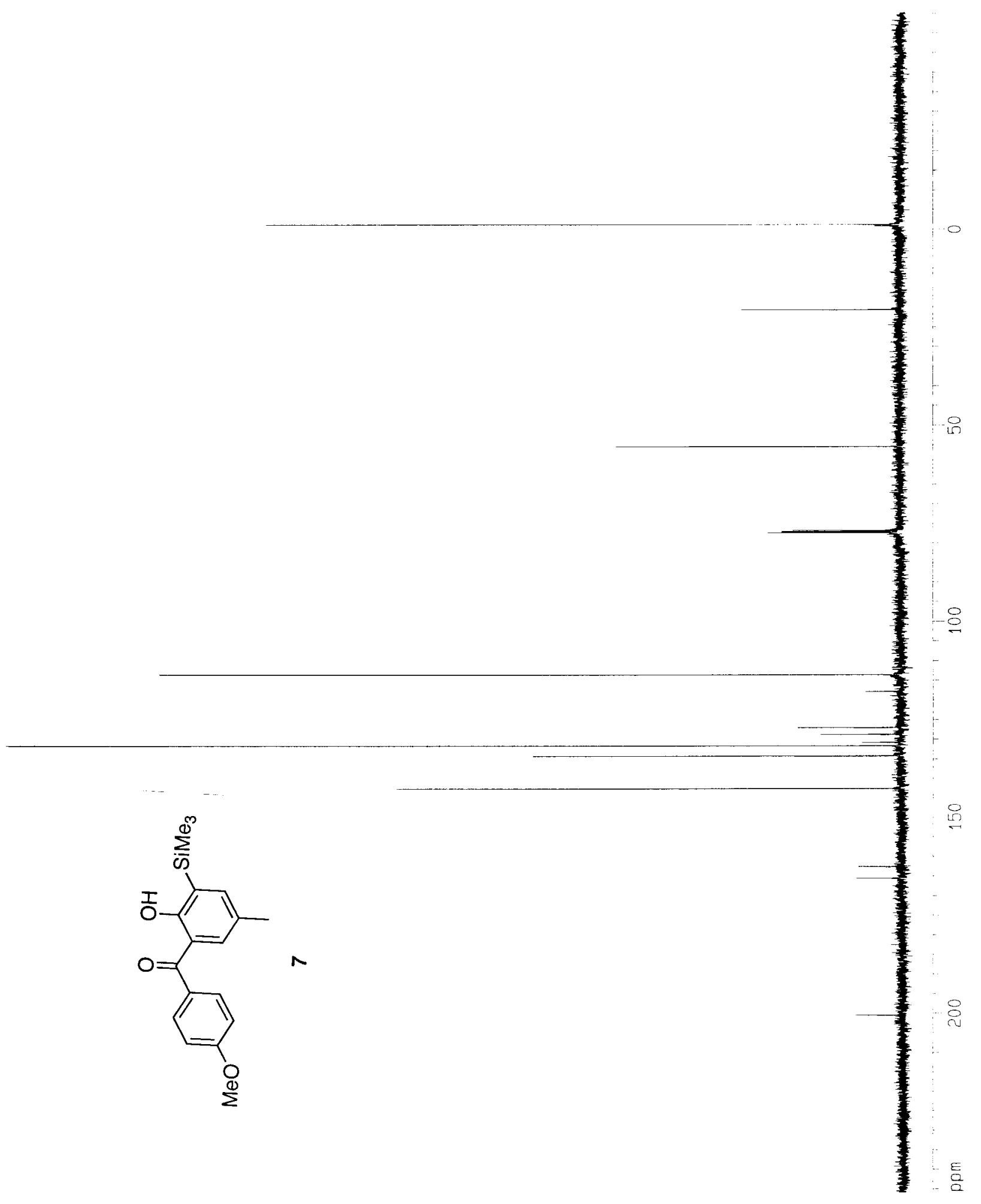




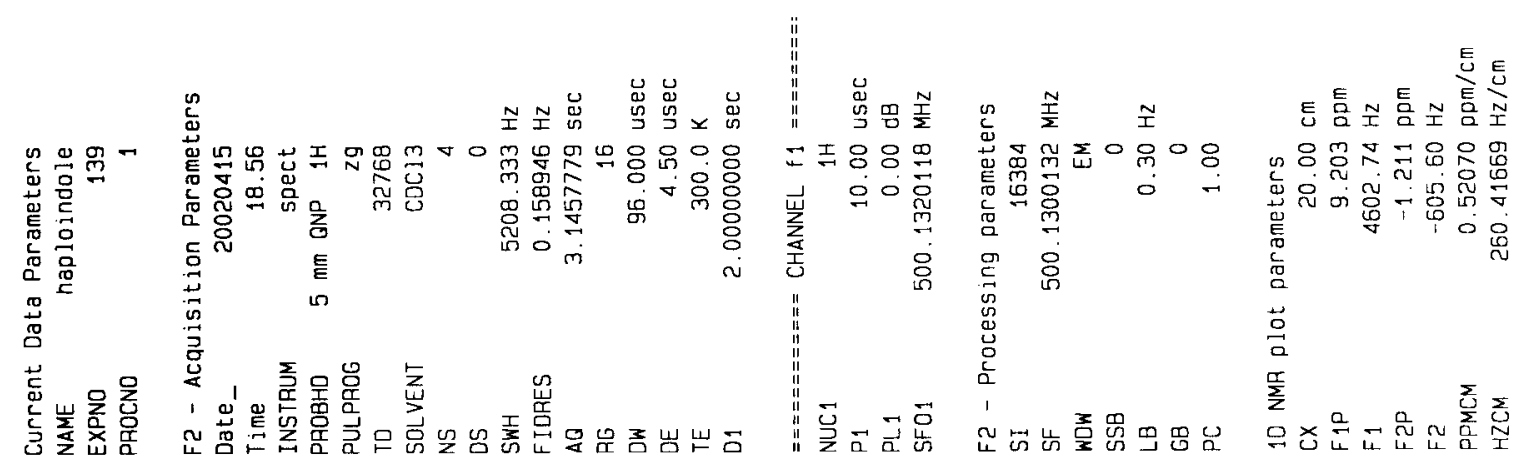

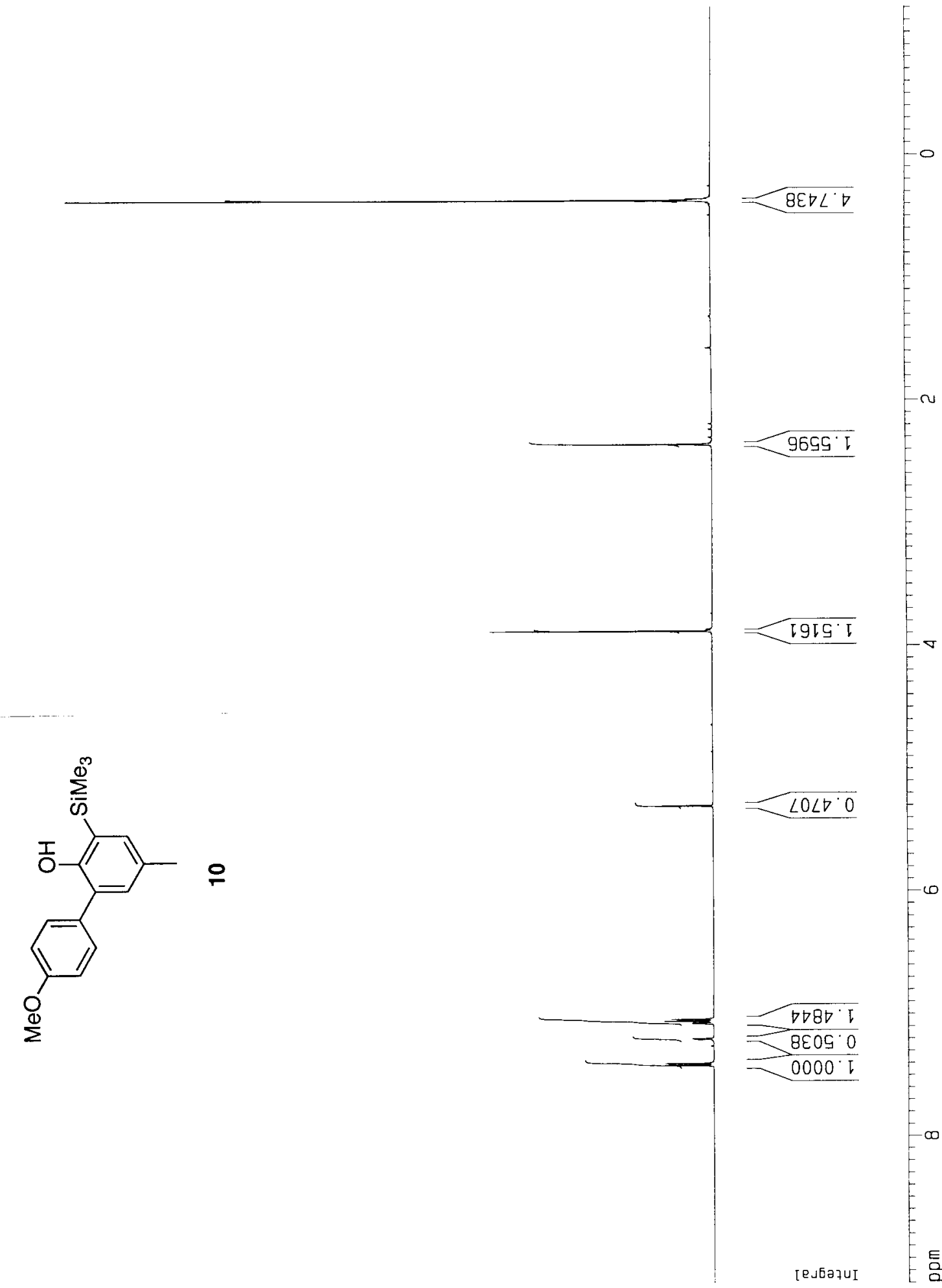



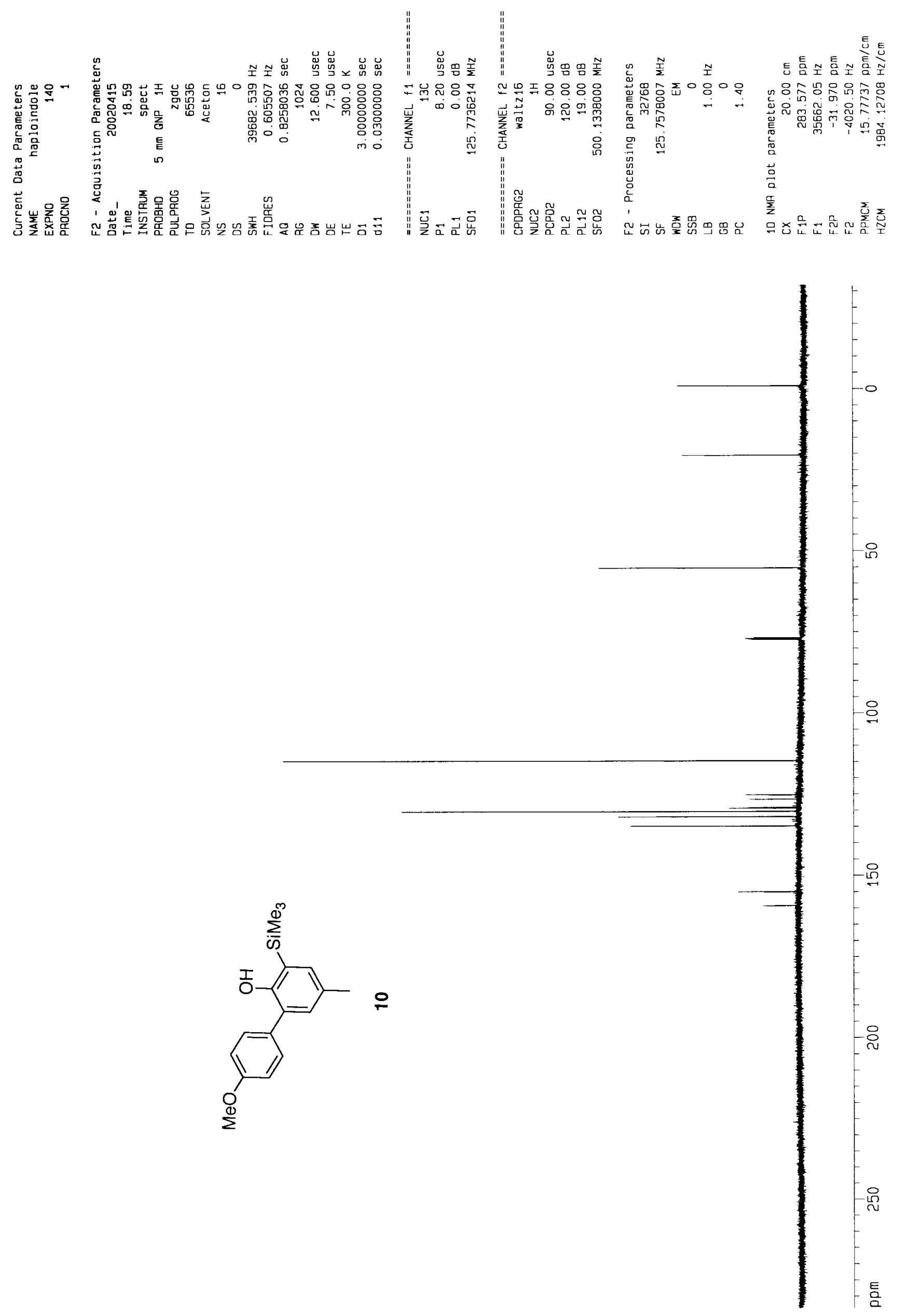


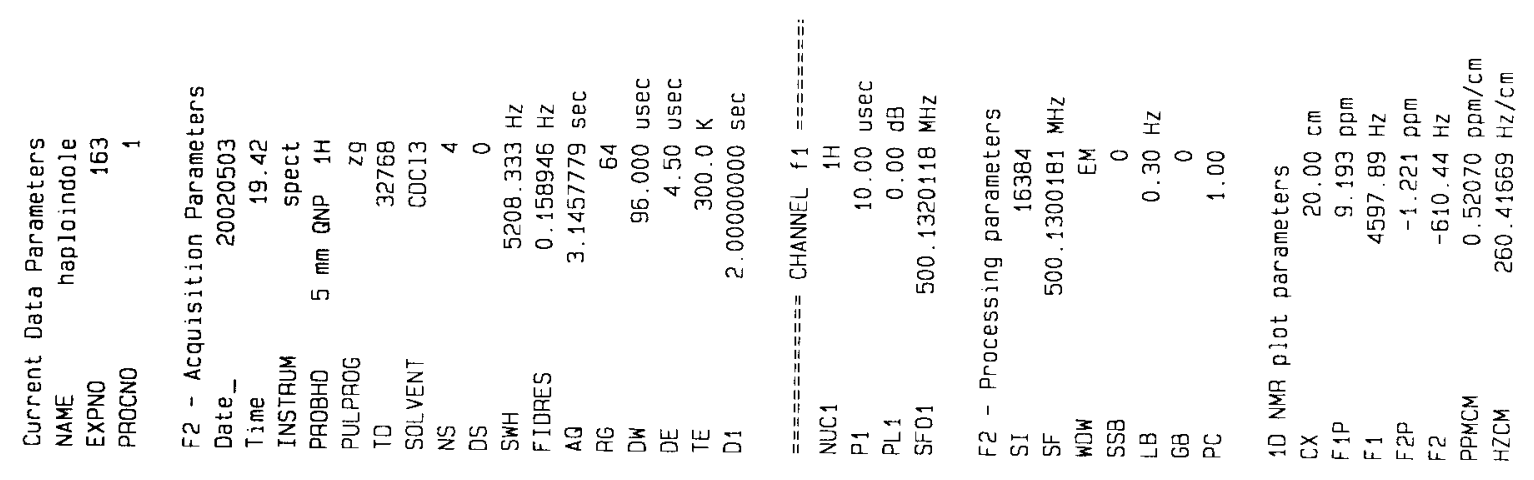

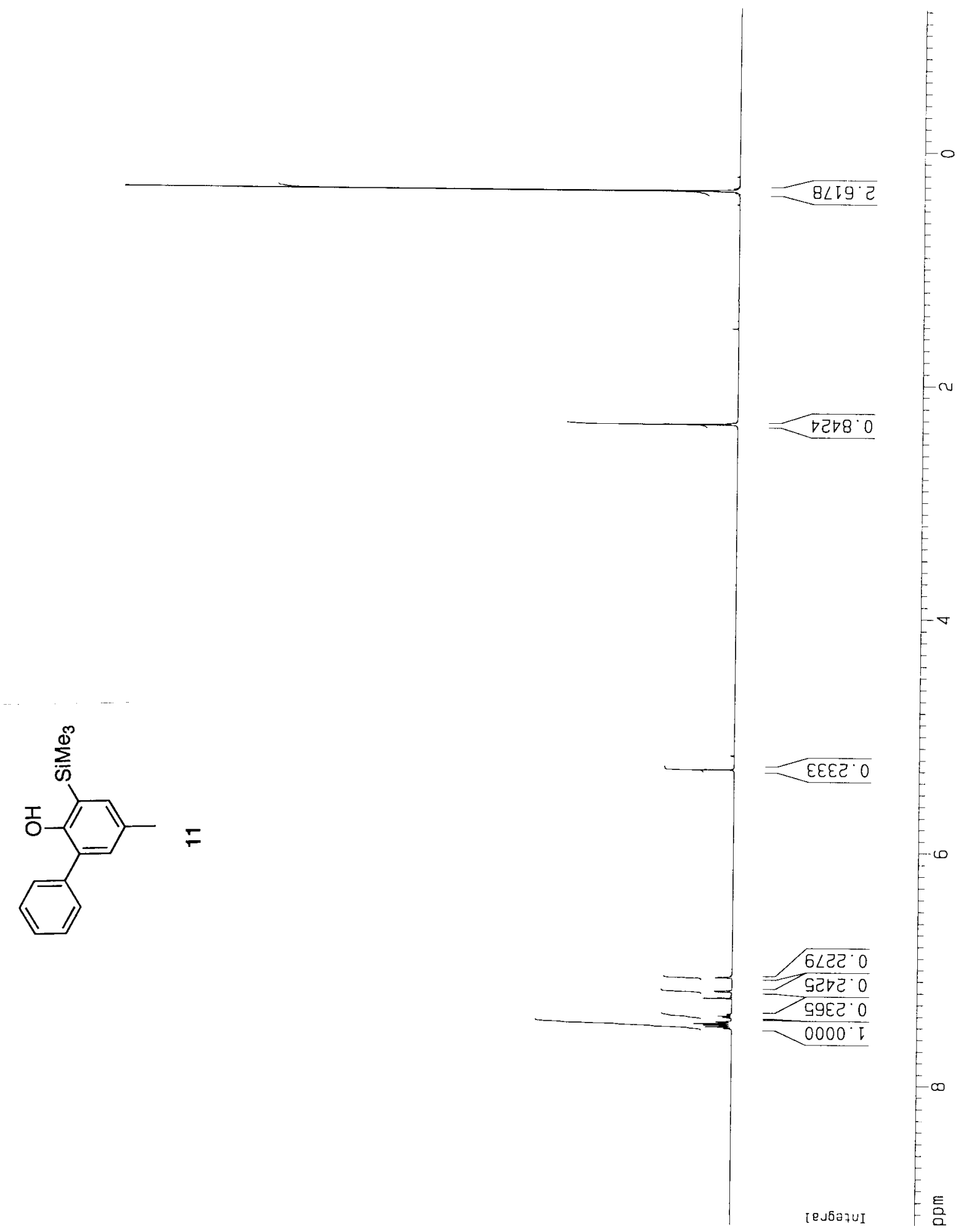




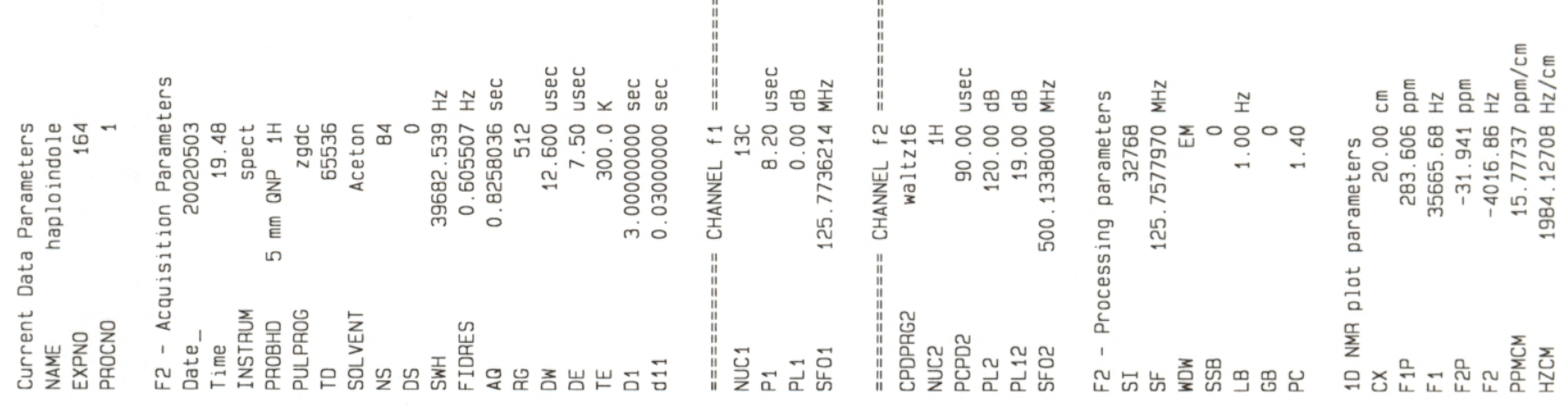

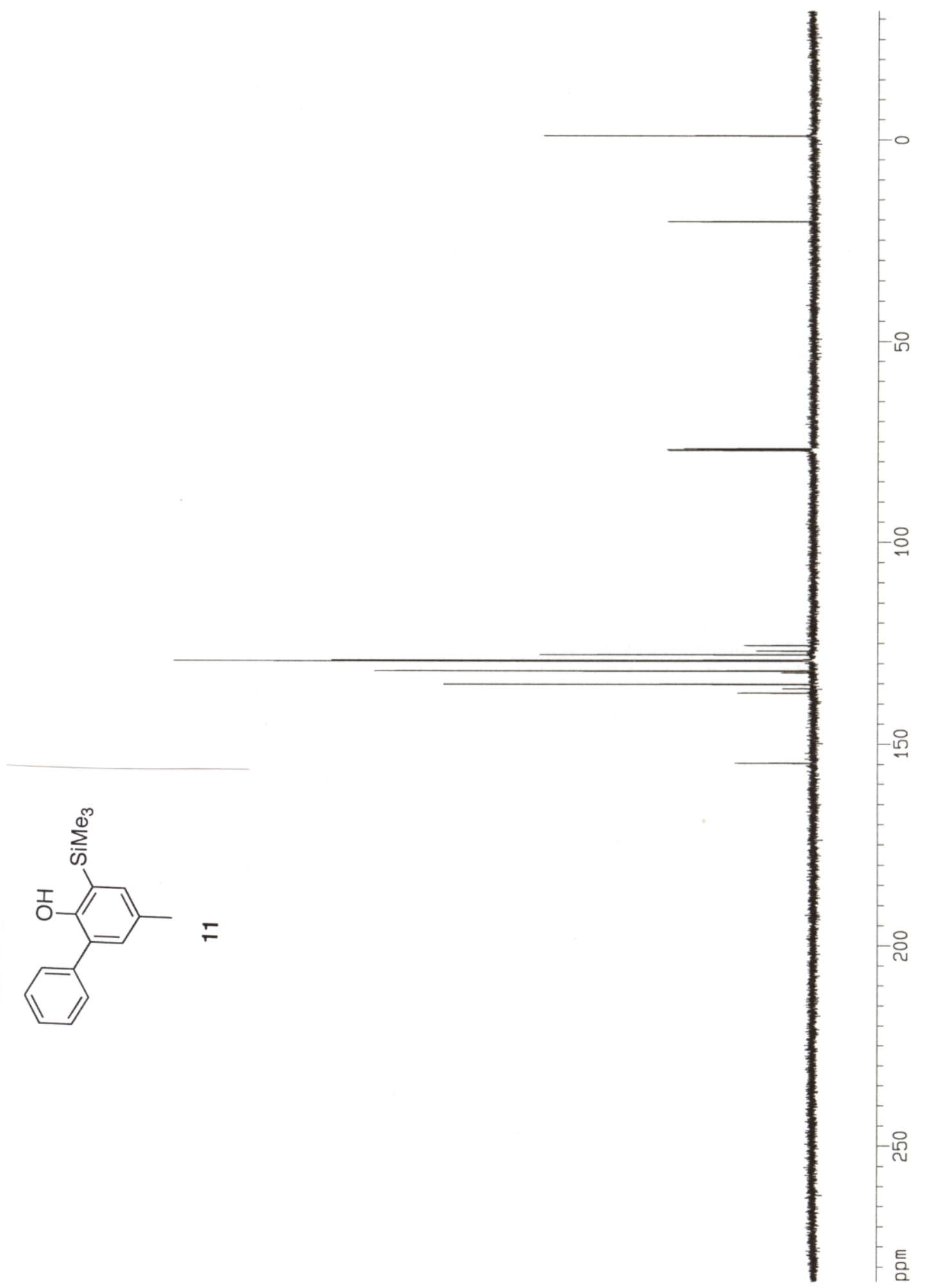

